\title{
SOBRE SISTEMAS DE EQUAÇÕES DIFERENCIAIS DE TIPO COOPERATIVO OU COMPETITIVO
}

\author{
YRMA ALEJANDRINA RAYMUNDO HUARUTO
}

\author{
DISSERTAÇÃO APRESENTADA \\ AO \\ INSTITUTO DE MATEMÁTICA E ESTATÍSTICA \\ DA \\ UNIVERSIDADE DE SÃO PAULO \\ PARA OBTENÇÃO DO GRAU DE MESTRE \\ EM \\ MATEMÁTICA APLICADA \\ ORIENTADOR :
}

Profa. Dra. ELVIA MUREB SALLUM

SÃO PAULO, JUNHO DE 1990. 
Agradecimentos

Desejo expressar os meus sinceros agradecimentos às pessoas e instituições que contribuíram para minha formação científica e para a elaboração do presente trabalho:

- À professora Dra. Elvia Mureb Sallum, pela sua paciente e dedicada orientação e valiosa colaboração na elaboração deste trabalho.

- Ao professor W. Muniz Oliva que me propôs o tema da dissertação e me apresentou à profa. Elvia M. S.

- Aos meus professores da USP que no decorrer dos anos fortaleceram a minha formação acadêmica. E à USP por me haver acolhido como aluna e como moradora do CRUSP durante vários anos de minha estadia no Brasil, até a conclusão dos meus estudos de mestrado. cluído.

- Ao CNPq e à CAPES pela valiosa ajuda econômica, que sem ela não teria con-

- À "Universidad Nacional de Ingenieria delPerú" onde trabalhei, por concederme a oportunidade de estudar no Brasil e pela ajuda econômica nos primeiros anos de estudo.

- Em geral a todos meus amigos que de uma ou outra maneira me incentivaram e fizeram acolhedora minha estadia no Brasil.

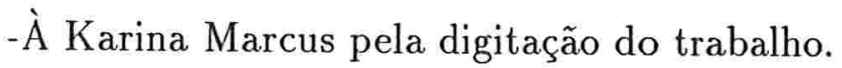

São Paulo, junho de 1990. 
Á:

- La memoria de mis padres Sabiniano y Victoria. - Mi familia querida del Perú, que siempre me dieron protección y cariño, confiando em mi retorno com éxito al seno familiar y al servicio de mi pais. 


\section{ÍNDICE}

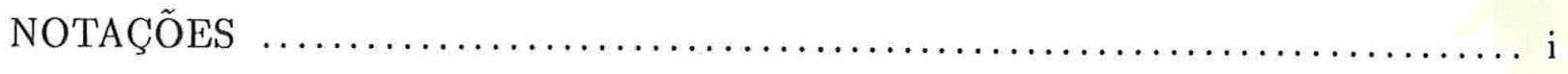

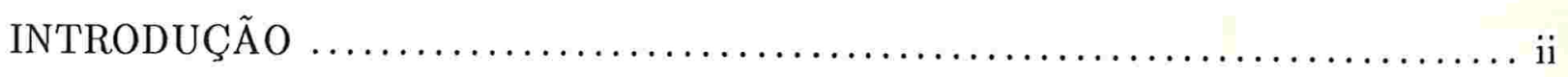

CAPÍTULO I. TEOREMA DE KAMKE - APLICAÇÃOES EM $\Re^{2}$. ........... 1

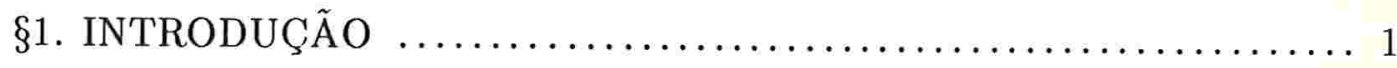

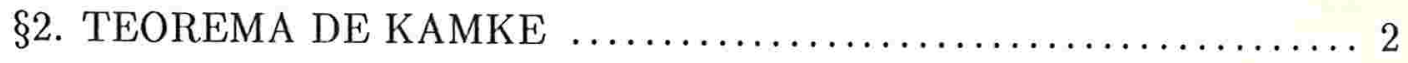

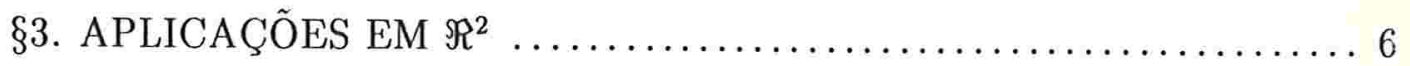

CAPÍTULO II. CONJUNTOS LIMITE DE SISTEMAS COOPERATIVOS OU COMPETITIVOS EM $\Re^{n}, n \geq 3 . \ldots \ldots \ldots \ldots \ldots \ldots \ldots \ldots$

§1. NÃO EXISTÊNCIA DE PONTOS RELACIONADOS NOS CONJUNTOS LIMITE $\ldots \ldots \ldots \ldots \ldots \ldots \ldots \ldots \ldots \ldots \ldots$

§2. COMPRESSIBILIDADE DO CONJUNTO LIMITE $\ldots \ldots \ldots \ldots \ldots 13$

§3. TRAJETÓRIAS COM PONTOS RELACIONADOS ............ 15

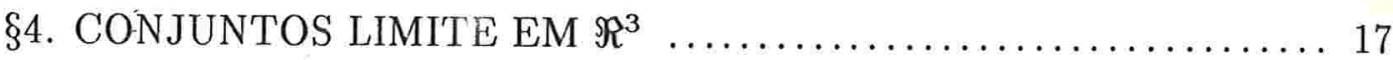

CAPÍTULO III. SISTEMAS̆ COOPERATIVOS IRREDUTÍVEIS. . . . . . . . . . 22

§1. FLUXOS COM DERIVADA POSITIVA $\ldots \ldots \ldots \ldots \ldots \ldots \ldots \ldots \ldots \ldots \ldots \ldots$

§2. RELAÇÃO ENTRE OS CONJUNTOS $\omega$-LIMITE DE PONTOS

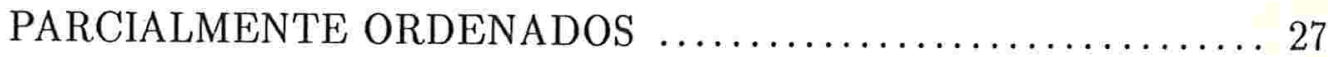

§3. TRAJETÓRIAS QUASE - CONVERGENTES ................ 32 CAPÍTULO IV. UM EXEMPLO DE COMPETIÇÃO ENTRE TRÊS ESPÉCIES .. 37

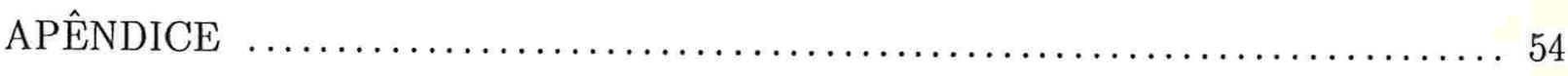

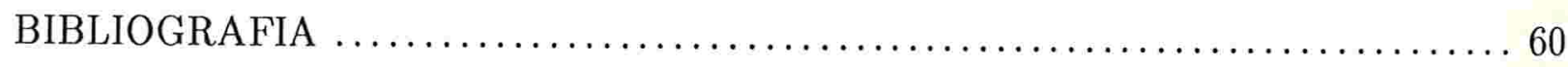




\section{NOTAÇÕES}

Sejam $x=\left(x_{1}, \ldots, x_{i}, \ldots, x_{n}\right), y=\left(y_{1}, \ldots, y_{i}, \ldots, y_{n}\right)$ pontos de $\Re^{n}$

$A=\left[a_{i j}\right], B=\left[b_{i j}\right]$ matrizes reais

$C, D$ subconjuntos não vazios de $\Re^{n}$.

$x<y \Leftrightarrow x_{i}<y_{i}$ para todo $i=1,2, \ldots, n$.

$x \leq y \Leftrightarrow x_{i} \leq y_{i}$ para todo $i=1,2, \ldots, n$.

* Quando $x<y$ ou $y<x$ diremos que $x$ e $y$ são pontos relacionados.

$A>B \Leftrightarrow a_{i j}>b_{i j}$ para todo $i, j$.

$A \geq B \Leftrightarrow a_{i j} \geq b_{i j}$ para todo $i, j$.

$C<D \Leftrightarrow c<d$ para todo $c \in C$ e todo $d \in D$.

$C \leq D \Leftrightarrow c \leq d$ para todo $c \in C$ e todo $d \in D$. 


\section{INTRODUÇÃO}

O presente trabalho, baseado principalmente nos artigos [7], [8] de Morris W. Hirsch e [13] de May - Leonard, tem como objetivo fundamental o estudo do comportamento limite das soluçóes de campos vetoriais de classe $C^{\mathbf{1}}$ em abertos de $\Re^{n}$, cujas matrizes jacobianas têm sempre todos os elementos fora da diagonal não negativos: campos cooperativos. Muitos dos resultados transferem-se automaticamente, por inversão do tempo, para os campos opostos cujas matrizes jacobianas têm sempre todos os elementos fora da diagonal não positivos (campos competitivos), como deixaremos claro no decorrer do trabalho.

Tais sistemas são geralmente usados para modelar fenômenos biológicos, químicos, etc. ([4], [13]). Assim, por exemplo, no capítulo IV estudaremos com detalhes um modelo biológico [13].

Veremos, entre outras propriedades, que os conjuntos $\alpha(\omega)$-limite tem uma posição especial dentro de $\Re^{n}$ o que influe para que estejam submetidos a certas restrições de caráter topológico.

Nesta introdução estaremos em geral tratando de campos cooperativos.

No início do capítuló I, veremos um resultado que, de certa maneira, sustentará quase toda a teoria desenv́olvida neste trabalho: o teorema de Kamke. Este teorema afirma que o fluxo mantém a ordem inicial, no sentido que $x(t) \leq y(t) \quad(x(t)<y(t))$ para $t \geq a$, se inicialmente para $t=a$ tivermos essa relação. A demonstração do caso $\leq$ foi adaptada de [2]. No caso < demonstramos usando idéias contidas em [12].

Uma conseqüência imediata do teorema de Kamke, que demonstraremos no capítulo I, é que a matriz do fluxo para $t \geq 0$ é sempre não negativa. Desta propriedade concluiremos que a dinâmica de campos cooperativos ou competitivos em $\Re^{2}$ é trivial: as órbitas não podem ser fechadas, nem apresentar oscilações e se forem limitadas, então convergem para um ponto de equilíbrio. Ao contrário de $\Re^{2}$, em $\Re^{n}, n \geq 3$, como veremos no exemplo do capítulo IV, para campos competitivos as ór'bitas podem convergir para ciclos e órbitas fechadas.

$\mathrm{Na}$ primeira parte do capítulo II mostramos uma propriedade que sobressai nos conjuntos $\alpha(\omega)$-limite para os dois tipos de campos em $\Re^{n}, n \geq 3$; eles não admitem pontos relacionados; portanto existem restrições para sua posição no espaço. Este resultado é, praticamente, uma conseqüência imediata do lema II.1.3, cuja demonstração original em [7] continha um erro que foi por nós superado; posteriormente o autor publicou em [9] a sua própria correção. Em exemplos do capítulo IV observamos que se cumpre a propriedade anterior: os conjuntos $\omega$-limite de pontos do int $\Re_{+}^{n}$ são órbitas fechadas contidas no simplexo $x_{1}+x_{2}+x_{3}=1$.

Mostramos, no capítulo II, dois teoremas centrais A e B, válidos para os dois tipos de campos. O teorema A afirma que os conjuntos $\alpha(\omega)$-limite projetam-se homeomorfi- 
camente em hiperplanos $E^{n-1}$ ortogonais a vetores positivos de $\Re^{n}$ e mais ainda, o fluxo em cada conjunto limite é conjugado ao fluxo em algum conjunto invariante de um campo localmente lipchitziano em $E^{n-1}$.

Neste mesmo capítulo mostramos, usando o teorema de Kamke, que órbitas positivas limitadas com pontos relacionados convergem para um ponto de equilíbrio. Observamos, como conseqüência imediata, que as órbitas periódicas não podem ter pontos relacionados, nem nós. Mais ainda mostramos que um campo cooperativo não pode ter órbitas periódicas atratoras.

Fundamentado no teorema A, o teorema B afirma que, em $\Re^{3}$, um conjunto $\alpha(\omega)$ limite compacto sem pontos de equilíbrio é uma órbita fechada ou um cilindro de órbitas fechadas; sendo uma única órbita no caso de $\omega$-limite de um campo cooperativo. A demonstração deste teorema ([7]) foi por nós melhorado no referente ao campo conjugado em $E^{2}$; fato que permitiu usar o teorema de Poincaré - Bendixson em $E^{2}$, para concluir que o conjunto limite é união de órbitas fechadas. Informalmente mostramos no capítulo III, que com uma condição adicional de irredutibilidade, o conjunto $\alpha$-limite de um sistema cooperativo é também uma órbita fechada [19].

O capítulo III trata de campos cooperativos irredutíveis que, como mostraremos, têm o fluxo fortemente monótono, para $t>0$, isto é: se $x \leq y, x \neq y$ então $x(t)<y(t)$. Para isto mostramos que a matriz derivada do fluxo para $t>0$ é positiva; superamos um erro contido na demonstração de [8] usando um método encontrado em [11].

Outro resultado mostrado no capítulo III é que os conjuntos $\omega$-limite de pontos $x \leq y, x \neq y$ são iguais, contendo somente pontos de equilíbrio, ou $\omega(x)<\omega(y)$. Evitamos uma aplicação não convincente do "Closing Lemma " na demonstração original dada em [8] seguindo a demonstração de [9]. Esta propriedade e idéias contidas em [10] foram usadas para demonstrar o resultado principal deste capítulo, o teorema C: "quase todas as órbitas positivas com fecho compacto aproximam-se do conjunto dos pontos de equilíbrio".

Agregando hipóteses adicionais ao teorema $\mathrm{C}$ : que os pontos de equilíbrio são hiperbólicos e o fluxo está definido para todo $t \geq 0$, mostramos que quase todas as trajetórias convergem para um poço.

Como já comentamos, no capítulo IV estudamos com detalhes, em $\Re^{3}$, o modelo de May-Leonard [13] de competição entre três espécies de população. Para uma variação de parâmetros, o $\omega$-limite de um ponto do int $\Re_{+}^{3}$ é uma órbita fechada contida em $x_{1}+$ $x_{2}+x_{3}=1$. Para outra variação de parâmetros o $\omega$-limite de qualquer ponto do int $\Re_{+}^{3}$ (exceto os da reta $x_{1}=x_{2}=x_{3}$ ) é um ciclo contendo três pontos de equilíbrio e contido na front $\Re_{+}^{3}$, cuja descrição melhoramos estudando [22]. Este modelo concretiza alguns resultados apresentados neste trabalho e acreditamos que tenham, entre outros exemplos, motivado a teoria geral apresentada nesta dissertação.

Finalmente, no apêndice, apresentamos dois teoremas pouco conhecidos. O teorema 1 refere-se à extensão de funções lipchitizianas e foi aplicado nas demonstrações dos 
teoremas A e B. O teorema 2, contido em [3], destaca um propriedade importante do fluxo dentro de um conjunto $\omega$-limite e foi aplicado na demonstração do teorema B e no modelo estudado no capítulo IV. 


\section{CAPÍTULO I . TEOREMA DE KAMKE - APLICAÇÕES EM $\Re^{2}$}

Neste capítulo apresentaremos o teorema de Kamke, que sustentará quase toda a teoria desenvolvida neste e nos próximos capítulos. Como aplicação daremos a dinâmica dos sistemas cooperativos ou competitivos bidimensionais.

\section{§1. INTRODUÇÃO.}

Consideraremos sistemas

$$
\dot{x}=F(x)
$$

onde $F=\left(F_{1}, \ldots, F_{n}\right): W \rightarrow \Re^{n}$ é um campo de classe $C^{1}$, definido num aberto $W \neq \emptyset$, $W \subset \Re^{n}$. Indicaremos com $x(t)=\Phi_{t}(x)$ a solução maximal de (I) tal que $x(0)=x$ com intervalo maximal $I$.

DEFINIÇÃO I.1.1 Dizemos que $F$ é cooperativo (competitivo) se $\frac{\partial F_{i}}{\partial x_{j}}(x) \geq 0\left(\frac{\partial F_{i}}{\partial x_{j}}(x) \leq 0\right)$ para $i \neq j$.

OBSERVAÇÃO I.1.2 Se o sistema $\dot{x}=F(x)$ é cooperativo, então o sistema $\dot{x}=-F(x)$ é competitivo e vice-versa.

\section{$\underline{\text { EXEMPLOS }}$}

1) Em [13] May - Leonard estudaram as equações

$$
\left\{\begin{array}{l}
\dot{x_{1}}=x_{1}\left(1-x_{1}-\alpha x_{2}-\beta x_{3}\right) \\
\dot{x_{2}}=x_{2}\left(1-\beta x_{1}-x_{2}-\alpha x_{3}\right) \\
\dot{x_{3}}=x_{3}\left(1-\alpha x_{1}-\beta x_{2}-x_{3}\right)
\end{array}\right.
$$

sobre $\Re_{+}^{3}=\left\{\left(x_{1}, x_{2}, x_{3}\right) \in \Re^{3}: x_{i} \geq 0, i=1,2,3\right\}$ onde $\alpha, \beta$ são reais positivos. Este é um sistema competitivo modelando competição de três espécies 1,2 e 3 cujas densidades são $x_{1}, x_{2}$ e $x_{3}$ respectivamente e que estudaremos com detalhes no capítulo IV.

2) J.S. Griffith [4] propõe o seguinte sistema para modelar um processo de controle celular.

$$
\left\{\begin{array}{l}
\dot{x_{1}}=\frac{x_{3}^{m}}{1+x_{3}^{m}}-\alpha x_{1} \\
\dot{x_{2}}=x_{1}-\beta x_{2} \\
\dot{x_{3}}=x_{2}-\gamma x_{3}
\end{array}\right.
$$

sobre $\Re_{+}^{3}$ onde $\alpha, \beta$ e $\gamma$ são parâmetros reais positivos e $m$ um inteiro positivo. Este é um sistema do tipo cooperativo. 
3) Sistemas competitivos dados por:

$$
\dot{x}_{i}=F_{i}(x)=x_{i} M_{i}(x) \quad i=1,2, \ldots, n
$$

onde $\frac{\partial M_{i}}{\partial x_{j}} \leq 0$ para $i \neq j$ em $\Re_{+}^{n}=\left\{x \in \Re^{n}: x_{i} \geq 0, i=1, \ldots, n\right\}$ são estudados por vários autores.

4) Fusco e W.Oliva em [16] estudaram campos de Jacobi $\dot{x}=F(x)$ onde $\frac{\partial F_{i}}{\partial x_{j}}>0$ se $|i-j|=1$ e $\frac{\partial F_{i}}{\partial x_{j}}=0$ se $|i-j|>1$.

E em [17] estudaram campos cíclicos $\dot{x}=F(x)$, onde $\frac{\partial F_{i}}{\partial x_{j}} \geq 0$ se $|i-j|=1$ ou $|i-j|=n-1$ $\mathrm{e} \frac{\partial F_{i}}{\partial x_{j}}=0$ se $1<|i-j| \neq n-1 \mathrm{com}$

$$
\frac{\partial F_{1}}{\partial x_{n}} \prod_{i=2}^{n} \frac{\partial F_{i}}{\partial x_{i-1}}+\frac{\partial F_{n}}{\partial x_{1}} \prod_{i=1}^{n-1} \frac{\partial F_{i}}{\partial x_{i+1}}>0
$$

\section{§2. TEOREMA DE KAMKE.}

Dados $x=\left(x_{1}, \ldots, x_{n}\right)$ e $y=\left(y_{1}, \ldots, y_{n}\right)$ dois pontos de $\Re^{n}$, dizemos que $x<y$ $(x \leq y)$ se e somente se $x_{i}<y_{i}\left(x_{i} \leq y_{i}\right)$ para todo $i=1, \ldots, n$.

DEFINIÇÃO I.2.1 Dizemos que um conjunto $V \subset \Re^{n}$ é p-convexo, se para todo $x, y$ em $V \operatorname{com} x \leq y$, o segmento de reta $\overline{x y}$ está inteiramente contido em $V$.

\section{TEOREMA I.2.2 (de Kamke)}

Sejam $V$ um aberto p-convexo de $\Re^{n}, \quad I$ um intervalo de $\Re, G=\left(G^{1}, \ldots, G^{n}\right): I \times V \rightarrow \Re^{n}$ uma aplicação contínua, com $\frac{\partial G^{i}}{\partial x_{j}}$ contínuas em $I \times V$ e $\frac{\partial G^{i}}{\partial x_{j}} \geq 0$ para $i \neq j$.

Sejam $x$ e $y:[a, b] \rightarrow V$ duas soluções do sistema $\dot{x}=G(t, x)$

i) Se $x(a) \leq y(a)$ (ou $x(a)<y(a)$ ) então $x(t) \leq y(t)$ para todo $t \in[a, b]$.

ii) Quando $V=\Re^{n}$, se $x(a)<y(a)$ então $x(t)<y(t)$ para todo $t \in[a, b]$.

\section{Demonstração.}

i) Seja $c=\sup \{t \in[a, b]: x(s) \leq y(s), s \in[a, t]\}$, mostraremos que $c=b$. 
Suponhamos que $c<b$. Evidentemente $c \geq a$.

Tomemos $\epsilon \in \Re^{n}, \epsilon>0$. Para cada $m \in Z^{+}$seja $y^{m}(t)$ a solução maximal com intervalo maximal $I_{m}$ do problema seguinte:

$$
\left\{\begin{array}{l}
\dot{y}=G(t, y)+\frac{1}{m} \epsilon=G_{m}(t, y) \\
y=y(c)+\frac{1}{m} \epsilon \text { em } t=c
\end{array}\right.
$$

Seja $\delta>0$ tal que $[c, c+\delta] \subset[a, b]$. Como $\lim _{m \rightarrow+\infty}\left(y(c)+\frac{1}{m} \epsilon\right)=y(c)$ e $y(t)$ está definida em $[c, c+\delta]$, então pelo teorema da continuidade com respeito às condições iniciais [18] existe $m_{0} \in Z^{+}$tal que para todo $m \geq m_{0}, I_{m} \supset[c, c+\delta]$ e $y^{m}$ converge uniformemente para $y$ em $[c, c+\delta]$.

Mostraremos que $x(t)<y^{m}(t)$ para todo $t \in[c, c+\delta]$ e $m \geq m_{0}$. Então tomando limite quando $m \rightarrow+\infty$ temos que $x(t) \leq y(t)$ para todo $t \in[c, c+\delta]$, o que contradiz a definição de $c$. Portanto $c=b$.

Suponhamos que não seja certo que $x(t)<y^{m}(t)$ para todo $t \in[c, c+\delta]$ e $m \geq m_{0}$. Então existem $m \geq m_{0}$ e $d \in(c, c+\delta]$ tais que $x(t)<y^{m}(t)$ para todo $t \in[c, d), x(d) \leq$ $y^{m}(d)$ e $x_{i}(d)=y_{i}^{m}(d)$ para algum $i$.

Como $V$ é p-convexo o segmento de reta com extremos $x(d)$ e $y^{m}(d)$ está contido em $V$. A restrição da aplicação $x \rightarrow G_{m}^{i}(d, x)$ sobre este segmento tem derivadas parciais contínuas; então pelo teorema do valor médio, existe um ponto $\xi$ no segmento aberto de extremos $x(d)$ e $y^{m}(d)$ tal que:

$$
G_{m}^{i}\left(d, y^{m}(d)\right)-G_{m}^{i}(d, x(d))=\sum_{j=1}^{n} \frac{\partial G_{m}^{i}}{\partial x_{j}}(d, \xi)\left(y_{j}^{m}(d)-x_{j}(d)\right) .
$$

Como $\frac{\partial G_{m}^{i}}{\partial x_{j}}(d, \xi) \geq 0$ para $i \neq j$ e $y_{i}^{m}(d)=x_{i}(d)$ temos que

$$
G_{m}^{i}\left(d, y^{m}(d)\right) \geq G_{m}^{i}(d, x(d))
$$

logo

$$
\dot{x}_{i}(d)<\dot{y}_{i}^{m}(d)
$$

já que $\dot{x}_{i}(d)=G^{i}(d, x(d))<G_{m}^{i}(d, x(d)) \leq G_{m}^{i}\left(d, y^{m}(d)\right)=\dot{y}_{i}^{m}(d)$.

Como

$$
\lim _{h \rightarrow 0^{+}} \frac{x_{i}(d)-x_{i}(d-h)}{h}<\lim _{h \rightarrow 0^{+}} \frac{y_{i}^{m}(d)-y_{i}^{m}(d-h)}{h}
$$

então existe $\sigma>0$ tal que para $0<h<\sigma$

$$
\frac{x_{i}(d)-x_{i}(d-h)}{h}<\frac{y_{i}^{m}(d)-y_{i}^{m}(d-h)}{h}
$$


assim $y_{i}^{m}(d-h)<x_{i}(d-h) \quad$ (já que $\left.x_{i}(d)=y_{i}^{m}(d)\right)$ o que contraria o fato $x(t)<y^{m}(t)$ para todo $t \in[c, d)$. Portanto $x(t)<y^{m}(t)$ para todo $t \in[c, c+\delta]$ e $m \geq m_{0}$.

ii) Supondo que $x(a)<y(a)$ e que $x(t) \nless y(t)$ para algum $t \in(a, b]$.

Então existem $i$ e $t_{1} \in(a, b]$ tais que $x(t)<y(t)$ para $t \in\left[a, t_{1}\right), x\left(t_{1}\right) \leq y\left(t_{1}\right)$ e $x_{i}\left(t_{1}\right)=y_{i}\left(t_{1}\right)$.

Seja

$$
\begin{gathered}
h(t)=y_{i}\left(a+t_{1}-t\right)-x_{i}\left(a+t_{1}-t\right) \text { com } t \in\left[a, t_{1}\right] \\
\dot{h}(t)=-\dot{y}_{i}\left(a+t_{1}-t\right)+\dot{x}_{i}\left(a+t_{1}-t\right)= \\
=G^{i}\left(a+t_{1}-t, x\left(a+t_{1}-t\right)\right)-G^{i}\left(a+t_{1}-t, y\left(a+t_{1}-t\right)\right) .
\end{gathered}
$$

Observamos que $x\left(a+t_{1}-t\right)<y\left(a+t_{1}-t\right)$ para $t \in\left(a, t_{1}\right]$, já que $s(t)=a+t_{1}-t \in\left[a, t_{1}\right)$.

$$
\begin{gathered}
\text { Como } x(s(t)) \leq y(s(t)) \text { para } t \in\left[a, t_{1}\right] \text { e } \frac{\partial G^{i}}{\partial x_{j}} \geq 0 \text { para } j \neq i, \text { tem-se que } \\
\qquad \begin{array}{c}
\dot{h}(t)=G^{i}\left(s(t), x(s(t))-G^{i}(s(t), y(s(t)) \leq\right. \\
\leq G^{i}\left(s(t), y_{1}(s(t)), \ldots, y_{i-1}(s(t)), x_{i}(s(t)), y_{i+1}(s(t)), \ldots, y_{n}(s(t))\right)-G^{i}(s(t), y(s(t)))= \\
=-\frac{\partial G^{i}}{\partial x_{i}}(s(t), \xi)\left(y_{i}(s(t))-x_{i}(s(t))\right) \leq \\
\leq\left|\frac{\partial G^{i}}{\partial x_{i}}(s(t), \xi)\right| h(t) \text { para } t \in\left[a, t_{1}\right]
\end{array}
\end{gathered}
$$

onde $\xi$ é um ponto do segmento aberto de extremos

$$
\begin{gathered}
\left(y_{1}(s(t)), \ldots, y_{i-1}(s(t)), x_{i}(s(t)), y_{i+1}(s(t)), \ldots, y_{n}(s(t))\right) \\
\text { e } \quad\left(y_{1}(s(t)), \ldots, y_{i}(s(t)), y_{i+1}(s(t)), \ldots y_{n}(s(t))\right) .
\end{gathered}
$$

Consideremos o seguinte conjunto compacto $K=\sigma(R)$, onde $R=\left[a, t_{1}\right] \times[0,1] \mathrm{e}$ $\sigma(s, t)=\left(s, y_{1}(s), \ldots, y_{i-1}(s),(1-t) x_{i}(s)+t y_{i}(s), y_{i+1}(s), \ldots, y_{n}(s)\right)$.

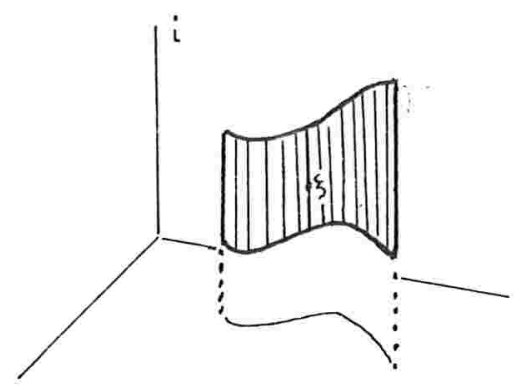




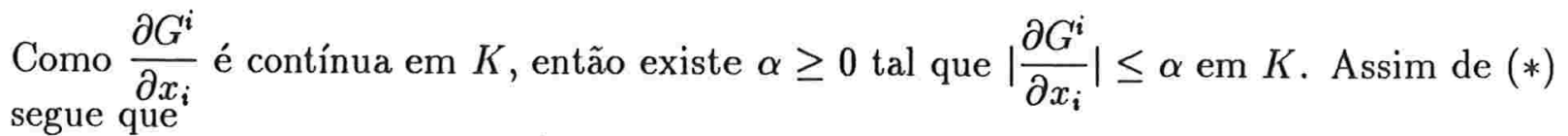

$$
\dot{h}(t) \leq \alpha h(t) \text { para } t \in\left[a, t_{1}\right]
$$

logo

$$
h(t) \leq \int_{a}^{t} \alpha h(r) d r \text { para } t \in\left[a, t_{1}\right]
$$

onde $\alpha \geq 0$ e $h(t) \geq 0$. Então pelo lema de Gronwall $h(t) \leq 0$; assim conclui-se que $h(t)=0$ para todo $t \in\left[a, t_{1}\right]$, de onde segue que $x_{i}(s)=y_{i}(s)$ para $s \in\left[a, t_{1}\right)$ o que contradiz o fato $x(t)<y(t)$ para $t \in\left[a, t_{1}\right)$. Portanto $x(t)<y(t)$ para todo $t \in[a, b]$.

\section{Notações:}

1) Sendo $A=\left[a_{i j}\right]$ uma matriz real, dizemos que

$A \geq 0$ se e somente se $a_{i j} \geq 0$ para todo $i, j$.

$A>0$ se e somente se $a_{i j}>0$ para todo $i, j$.

Uma conseqüência imediata do teorema de Kamke é dada na seguinte proposição.

PROPOSIÇÃO I.2.3 Se (I) é um sistema cooperativo, então para $x \in W$ e $t>0, t \in I$, tem-se $D \Phi_{t}(x) \geq 0$.

Demonstração. Consideremos a seguinte equação matricial

$$
\dot{M}=A(t) M=G(t, M)
$$

onde $A(t)=D F\left(\Phi_{t}(x)\right)$ e $M=\left[M_{i j}\right]$ é uma matriz real de ordem $n \times n$.

$M(t)=D \Phi_{t}(x)$ é solução desta equação matricial com $M(0)=$ Id. onde Id. é a matriz identidade e $N(t) \equiv 0$ é a solução nula.

Como $N(0) \leq M(0)$, pelo teorema de Kamke temos que $N(t) \leq M(t)$ para todo $t \geq 0, t \in I$. Observemos que

$$
G_{i k}(t, M)=\sum_{j=1}^{n} \frac{\partial F_{i}}{\partial x_{j}}\left(\Phi_{t}(x)\right) M_{j k}
$$

$\mathrm{e}$

$$
\frac{\partial G_{i k}}{\partial M_{r s}}(t, M)=\left\{\begin{array}{cl}
\frac{\partial F_{i}}{\partial x_{r}}\left(\Phi_{t}(x)\right) \geq 0 & \text { para } i \neq r, s=k \\
0 & \text { para } s \neq k
\end{array}\right.
$$

portanto $\frac{\partial G_{i k}}{\partial M_{r s}}(t, M) \geq 0$ para $(i, k) \neq(r, s)$. 
Como aplicação desta proposição mostraremos que sistemas cooperativos ou competitivos bidimensionais têm dinâmica trivial.

\section{§3. APLICAÇÕES EM $\Re^{2}$.}

PROPOSIÇÃO I.3.1 Seja $W$ um aberto de $\Re^{2}, F: W \rightarrow \Re^{2}$ um campo de classe $C^{1}$, cooperativo ou competitivo e $x(t)=\left(x_{1}(t), x_{2}(t)\right)$ a solução maximal do problema $\left\{\begin{array}{l}\dot{x}=F(x) \\ x(0)=x\end{array}\right.$ definida no intervalo $I$. Então $I$ é união de dois intervalos $J$ e $K$ iguais ou disjuntos tais que cada componente $x_{i}(t)$ é monótona em cada uma delas.

Demonstração. Basta demonstrar para o caso cooperativo, pois se $F$ é competitivo, então $-F$ é cooperativo e por inversão do tempo se conclui para o caso competitivo.

Suporemos que $x(t)$ não seja constante. Consideremos:

$$
\begin{aligned}
& I_{1}=\left\{t \in I \mid F_{i}(x(t)) \geq 0, i=1,2\right\}, \\
& I_{2}=\left\{t \in I \mid F_{2}(x(t))>0>F_{1}(x(t))\right\}, \\
& I_{3}=\left\{t \in I \mid F_{i}(x(t)) \leq 0, i=1,2\right\}, \\
& I_{4}=\left\{t \in I \mid F_{2}(x(t))<0<F_{1}(x(t))\right\}
\end{aligned}
$$

então $I=\bigcup_{i=1}^{4} I_{i}, I_{i} \cap I_{j}=\emptyset$ para todo $i \neq j$.

i) Se existir $t_{1} \in I_{1}\left(I_{3}\right)$, então para todo $t \in I, t \geq t_{1}$ tem-se $t \in I_{1}\left(I_{3}\right)$ pois

$$
D \Phi_{t-t_{1}}\left(x\left(t_{1}\right)\right) F\left(x\left(t_{1}\right)\right)=F(x(t)) \text { e } D \Phi_{t-t_{1}}\left(x\left(t_{1}\right)\right) \geq 0
$$

ii) Se existir $t_{1} \in I_{2}\left(I_{4}\right)$, então para todo $t \in I, t \leq t_{1}$ tem-se $t \in I_{2}\left(I_{4}\right)$ pois se $t \in I, t<t_{1}$ pelo caso i) $t \in I_{2} \cup I_{4}$ e como $F(x(t)) \neq 0$, então $F(x(t))$ fica no mesmo quadrante que $F\left(x\left(t_{1}\right)\right)$.

Assim podemos concluir o teorema das seguintes observações:

a) Se $0 \in I_{1}\left(I_{3}\right)$ e existe $t_{1} \in I_{2}\left(I_{4}\right)$ (portanto $t_{1}<0$ ), tomando $t_{0}=\min \{t \mid t \in$ $\left.I_{1}\left(I_{3}\right)\right\}$, tem-se de i) e ii) que $t \in I_{2}\left(I_{4}\right)$ para todo $t \in I, t<t_{0}$.

b) Se $0 \in I_{2}\left(I_{4}\right)$ e existe $t_{2} \in I_{1}\left(I_{3}\right)$ (portanto $t_{2}>0$ ), tomando $t_{0}=\min \{t \mid t \in$ 
$\left.I_{1}\left(I_{3}\right)\right\}$, tem-se de i) e ii) que $t \in I_{2}\left(I_{4}\right)$ para todo $t \in I, t<t_{0}$.
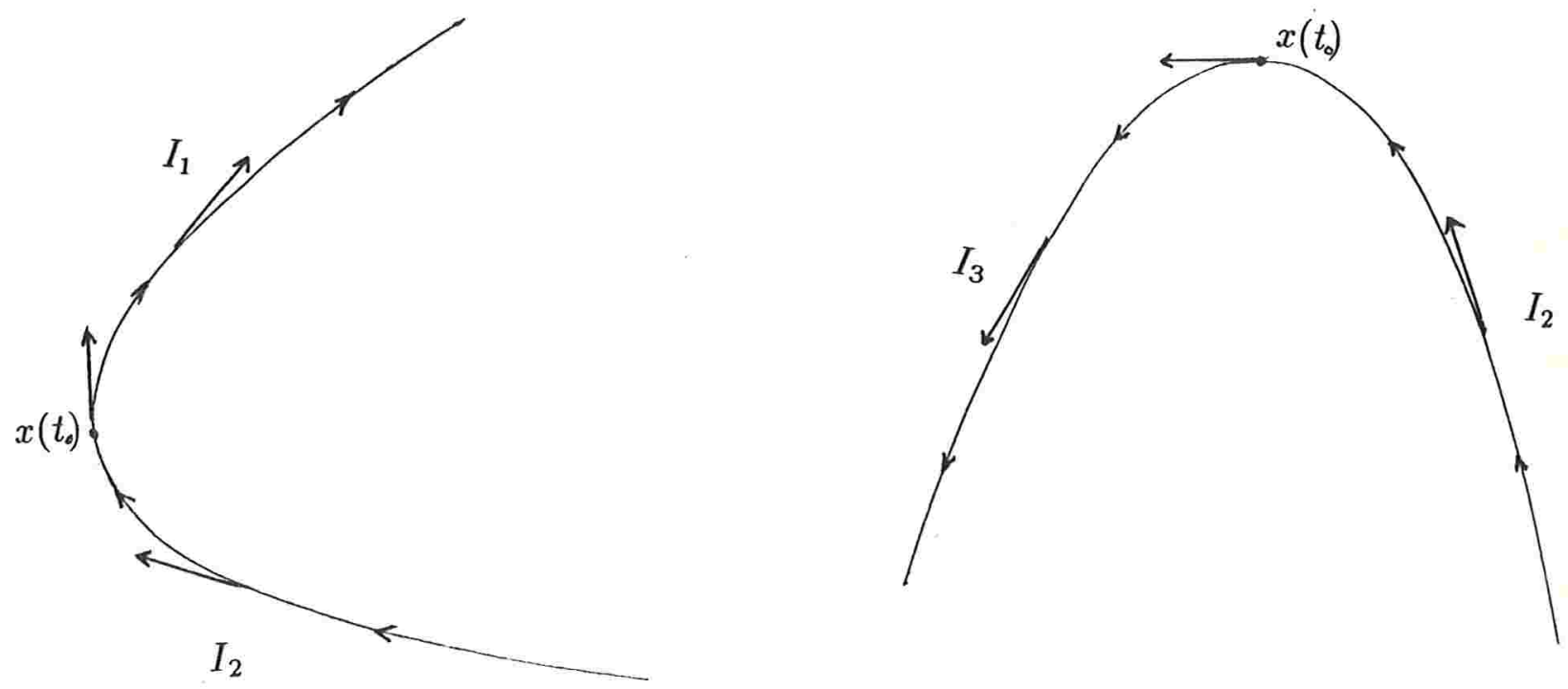

Os seguintes corolários seguem facilmente:

COROLÁRIO I.3.2 Nas mesmas hipóteses da proposição I.3.1, se $x(t)$ é uma solução limitada para $t \geq 0(t \leq 0)$, então $x(t)$ converge para algum ponto quando $t \rightarrow+\infty$ $(t \rightarrow-\infty)$.

COROLÁRIO I.3.3 Nas mesmas hipóteses da proposição I.3.1 o sistema não tem órbitas periódicas não triviais.

Como na demonstração da proposição I.3.1 segue a seguinte: 
OBSERVAÇÃO I.3.4 Seja $\dot{x}=F(x)$ um sistema cooperativo onde $F$ é um campo de classe $C^{1}$ num aberto $W$ de $\Re^{n}$ com uma solução $x(t)=\left(x_{0}(t), \ldots, x_{n}(t)\right)$ definida em $[0,+\infty)$ e tal que $x(0)=x_{0}$. Se $F\left(x_{0}\right) \geq 0\left(F\left(x_{0}\right) \leq 0\right)$ então, todas as $x_{i}(t)$ são não decrescentes (não crescentes). Se, além disso, $x(t)$ for limitada, então converge. 


\section{CAPÍTULO II. CONJUNTOS LIMITE DE SISTEMAS}

\section{COOPERATIVOS OU COMPETITIVOS EM $\Re^{n}, n \geq 3$.}

Apresentamos dois teoremas centrais: A e B. O teorema A destaca propriedades referentes ao caráter geométrico dos conjuntos limites de sistemas cooperativos ou competitivos em geral em $\Re^{n}$. O teorema B corresponde a um análogo ao teorema de Poincaré - Bendixson no plano.

\section{§1. NÃO EXISTÊNCIA DE PONTOS RELACIONADOS NOS CONJUNTOS LIMITE}

DEFINIÇÃO II.1.1 Diremos que dois pontos $p, q \in \Re^{n}$ estão relacionados se $p<q$ ou $q<p$.

DEFINIÇÃO II.1.2 Seja $y:[a, b] \rightarrow \Re^{n}$ uma curva em $\Re^{n}$. O intervalo $[a, b]$ é chamado ascendente (descendente) se $y(a)<y(b)(y(b)<y(a))$.

LEMA II.1.3 Se (I) é um sistema cooperativo ou competitivo num aberto p-convexo $W$ de $\Re^{n}$, então nenhuma solução tem um intervalo ascendente e um descendente disjuntos.

Demonstração. Demonstraremos para o caso cooperativo; o caso competitivo segue por inversão do tempo.

Suponhamos que existam ambos, um intervalo ascendente $K=[s, b]$ e um intervalo descendente disjuntos $J=[a, r]$ tais que $a<r<s<b$.

Sempre existe um intervalo ascendente $K^{\prime}=\left[s^{\prime}, b\right] \subset K$ tal que para todo $t \in$ $K^{\prime}, t \neq s^{\prime}, y(t) \not \leq y\left(s^{\prime}\right)$. De fato: supondo que exista $t \in K, t \neq s$ tal que $y(t) \leq y(s)$, basta tomar $s^{\prime}=\sup \{t \in[s, b]: y(t) \leq y(s)\}$ e como $y\left(s^{\prime}\right) \leq y(s)<y(b)$ tem-se $s^{\prime}<b$; caso contrário $K=K^{\prime}$.

a) Se $r-a \leq b-s^{\prime}$ :

transladamos o intervalo descendente $[a, r]$ ao intervalo $\left[s^{\prime}, s^{\prime}+r-a\right] \subset K^{\prime}$, então, pelo teorema de Kamke temos que $y\left(s^{\prime}+r-a\right) \leq y\left(s^{\prime}\right)$. Logo existe $t^{\prime}=s^{\prime}+r-a \in\left(s^{\prime}, b\right]$ tal 
que $y\left(t^{\prime}\right) \leq y\left(s^{\prime}\right)$, o que contradiz a definição de $K^{\prime}$.

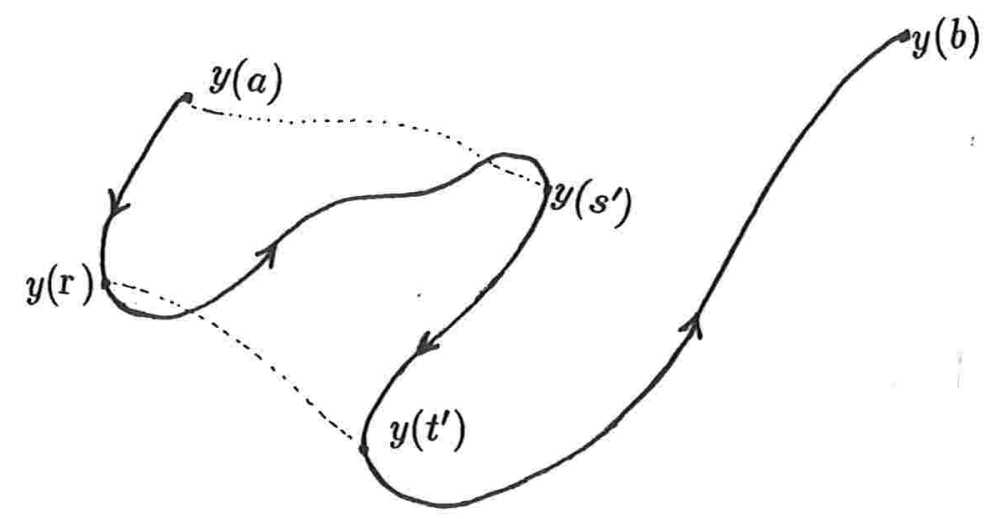

b) $\underline{\text { Se } r-a>b-s^{\prime}}$ :

transladamos o intervalo $[a, r]$ ao intervalo $[a+b-r, b]$, então $y(b) \leq y(a+b-r)$ e como $y\left(s^{\prime}\right) \leq y(s)<y(b)$ segue-se que $y\left(s^{\prime}\right)<y(a+b-r)$;

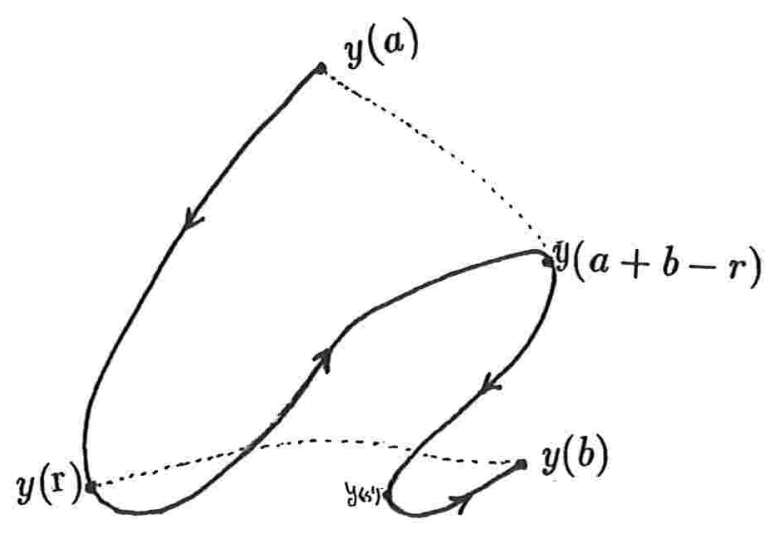

i) Se $s^{\prime}-(a+b-r) \leq b-s^{\prime}$, então como no caso a) transladamos o intervalo descendente $\left[a+b-r, s^{\prime}\right]$ ao intervalo $\left[s^{\prime}, 2 s^{\prime}-(a+b-r)\right] \subset K^{\prime}$, então $y\left(2 s^{\prime}-(a+b+r)\right) \leq y\left(s^{\prime}\right)$, logo existe $\bar{t}=2 s^{\prime}-(a+b-r)$ tal que $\bar{t} \in\left(s^{\prime}, b\right]$ e $y(\bar{t}) \leq y\left(s^{\prime}\right)$ o que contradiz a definição 
de $K^{\prime}$.

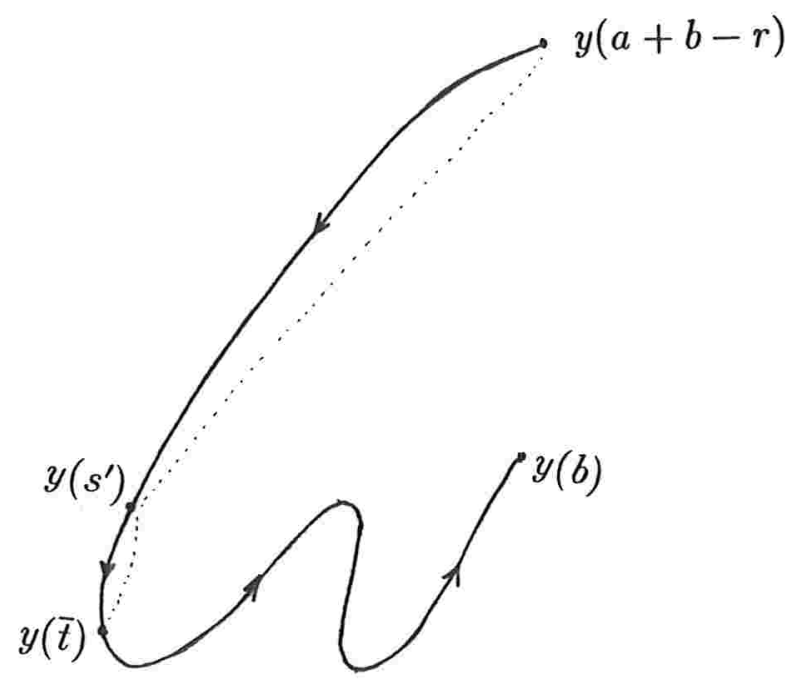

ii) Se $s^{\prime}-(a+b-r)>b-s^{\prime}$, então transladamos o intervalo descendente $\left[a+b-r, s^{\prime}\right]$ ao intervalo $\left[a+b-r+\left(b-s^{\prime}\right), b\right]$. Como $y\left(s^{\prime}\right)<y(b) \leq y(\hat{t})$ tem-se $y\left(s^{\prime}\right)<y(\hat{t})$ e portanto $\left[\hat{t}, s^{\prime}\right]$ é um intervalo descendente, onde $\hat{t}=a+b-r+\left(b-s^{\prime}\right)$.

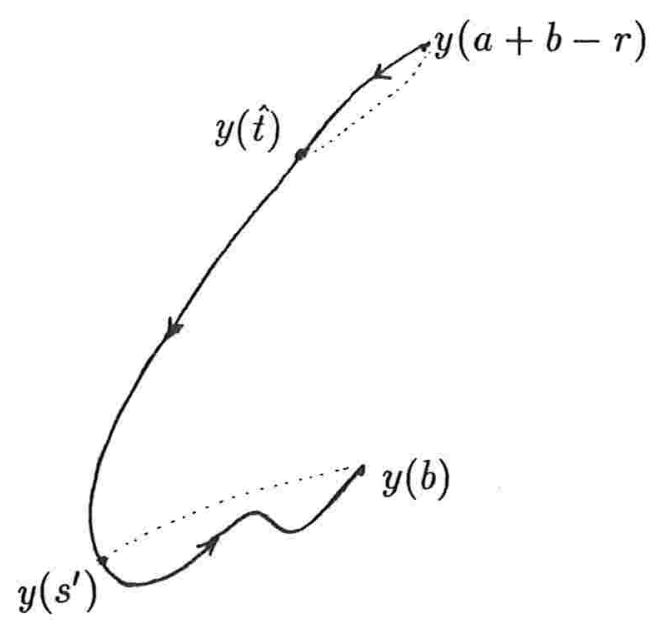

Se $s^{\prime}-\hat{t} \leq b-s^{\prime}$ caímos no caso i), caso contrário repetimos o processo ii) para os intervalos $\left[\hat{t}, s^{\prime}\right]$ e $\left[s^{\prime}, b\right]$ e assim continuamos sucessivamente até encontrar um $\hat{t}$ tal que $s^{\prime}-\hat{t} \leq b-s^{\prime}$, isto é, até cair no caso i).

DEFINIÇÃO II.1.4 O conjunto $\omega$-limite $\omega(x)$ da solução $x(t)$ de (I) é o conjunto de pontos $p \in W$ tais que $p=\lim _{t_{k} \rightarrow+\infty} x\left(t_{k}\right)$ para alguma seqüência $t_{k} \rightarrow+\infty$. O conjunto $\alpha$-limite $\alpha(x)$ é definido similarmente com $t_{k} \rightarrow-\infty$. 
Observamos que se $\omega(x)$ consiste de um único ponto $p$, então $x(t)$ converge para $p$ e este ponto é necessariamente um equilíbrio.

Por um conjunto limite entenderemos um conjunto $\alpha$-limite ou $\omega$-limite de algum ponto.

PROPOSIÇÃO II.1.5 Nas mesmas hipóteses do lema II.1.3 dois pontos de um conjunto limite $L$ não estão relacionados. Além disso se $y \in L$, então $F(y)$ não está relacionado com o vetor nulo.

Demonstração. Sem perda de generalidade consideremos $L=\omega(x)$.

Suponhamos que existam dois pontos $p, q$ em $\omega(x)$ tais que $p<q$. Como $p=$ $\lim _{t_{k} \rightarrow+\infty} x\left(t_{k}\right), q=\lim _{s_{j} \rightarrow+\infty} x\left(s_{j}\right)$, então existe $n_{0} \in N$ tal que para todo $k, j \geq n_{0}, x\left(t_{k}\right)<$ $x\left(s_{j}\right)$. Assim, existem $t<s<s^{\prime}<t^{\prime}$ tais que $x(t)<x(s)$ e $x\left(t^{\prime}\right)<x\left(s^{\prime}\right)$; então a solução $x(t)$ tem um intervalo ascendente $[t, s]$ e um intervalo descendente $\left[s^{\prime}, t^{\prime}\right]$ disjuntos, o que contradiz o lema II.1.3.

Por outro lado, suponhamos que exista $y \in \omega(x)$ tal que $F(y)>0$.

Consideremos a solução $y(t)=\left(y_{1}(t), \ldots, y_{n}(t)\right)$ tal que $y(0)=y$; como $\dot{y}_{i}(0)=F_{i}(y)>0$ para todo $i=1,2, \ldots, n$, então cada $y_{i}(t)$ é crescente numa vizinhança de $t=0$. Assim, existe $\bar{t}>0$ tal que $y(\bar{t})>y(0)=y$, como $y(\bar{t}) \in \omega(x)$ contrariamos o resultado anterior. $\square$

Uma aplicação da proposição II.1.5 é dada abaixo

APLICAÇÃO II.1.6 Formulamos e provamos heuristicamente a seguinte propriedade: (formalmente e mais geralmente tratado em [7]).

"Num aberto p-convexo $W \subset \Re^{3}$, um campo $F$ cooperativo ou competitivo não admite duas órbitas fechadas $\mathcal{O}_{1}$ e $\mathcal{O}_{2}$ encadeadas."

De fato: supondo que existissem duas órbitas fechadas encadeadas $\mathcal{O}_{1}$ e $\mathcal{O}_{2}$, então existiriam pontos $p, \bar{p}$ em $\mathcal{O}_{1}$ e $q, \bar{q}$ em $\mathcal{O}_{2}$ tais que $q<p$ e $\bar{p}<\bar{q}$.

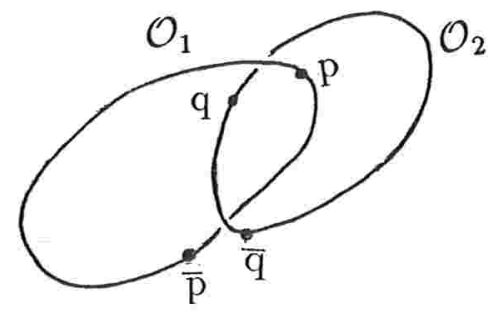


Mostraremos que alguma $\mathcal{O}_{i}$ tem dois pontos relacionados, o que contraria a proposição II.1.5.

Suponhamos que $F$ seja cooperativo.

Se $p=p(0)$ e $\bar{p}=p\left(t_{1}\right)$ com $t_{1}>0$, como $q<p$, então pelo teorema de Kamke tem-se que $q\left(t_{1}\right)<p\left(t_{1}\right)=\bar{p}<\bar{q}$, o que contraria a proposição II.1.5.

Se $\bar{p}=\bar{p}(0)$ e $p=\bar{p}\left(t_{1}\right)$ com $t_{1}>0$, como $\bar{p}<\bar{q}$ pelo teorema de Kamke tem-se que $q<p=\bar{p}\left(t_{1}\right)<\bar{q}\left(t_{1}\right)$ o que contraria a proposição II.1.5.

Se $F$ é um campo competitivo, basta considerar o campo cooperativo $-F$.

\section{§2. COMPRESSIBILIDADE DO CONJUNTO LIMITE}

O teorema a seguir explicita de uma certa maneira a "dimensão máxima" e a posição relativa dos conjuntos limites de sistemas cooperativos ou competitivos, fatos esses que estão subentendidos na proposição II.1.5.

TEOREMA A Dado um vetor $v \in \Re^{n}, v>0$, se $L$ é um conjunto limite de um sistema (I) cooperativo ou competitivo em $W$ p-convexo, $E^{n-1}$ um hiperplano ortogonal a $v$ e $\pi: \Re^{n} \rightarrow E^{n-1}$ a projeção ortogonal, então:

i) $\left.\pi\right|_{L}$ é um homeomorfismo com inversa $\left(\left.\pi\right|_{L}\right)^{-1}$ lipchitziana.

ii) $\pi$ leva, respeitando parametrização, as trajetórias de $F$ em $L$ nas trajetórias de um campo $H$ definido em $\pi(L)$ e localmente lipchitiziano.

Neste caso dizemos que L é compressível ao longo do vetor positivo $v$.

\section{Demonstração.}

i) Supondo que $\left.\pi\right|_{L}$ não seja injetora: existem $p, q \in L, p \neq q$ tais que $\left.\pi\right|_{L}(p)=$ $\left.\pi\right|_{L}(q)$. Assim $p=\left.\pi\right|_{L}(p)+s v, q=\left.\pi\right|_{L}(q)+t v$, com $s, t \in \Re$ e, portanto, $p>q$ ou $p<q$ conforme $s-t>0$ ou $s-t<0$, o que contraria a proposição II.1.5.

Supondo que $\left(\left.\pi\right|_{L}\right)^{-1}$ não seja lipchitziana: para todo $n>0, n \in N$ existem $a_{n}, b_{n}$ em $\pi(L)$ tais que

$$
\left|\left(\left.\pi\right|_{L}\right)^{-1}\left(a_{n}\right)-\left(\left.\pi\right|_{L}\right)^{-1}\left(b_{n}\right)\right|>n\left|a_{n}-b_{n}\right| .
$$

Sendo

$$
\begin{aligned}
& p_{n}=\left(\left.\pi\right|_{L}\right)^{-1}\left(a_{n}\right)=a_{n}+t_{n} \frac{v}{|v|} \\
& q_{n}=\left(\left.\pi\right|_{L}\right)^{-1}\left(b_{n}\right)=b_{n}+s_{n} \frac{v}{|v|}
\end{aligned}
$$


tem-se

$$
\left|\lambda_{n}\right|=\sqrt{\left|p_{n}-q_{n}\right|^{2}-\left|a_{n}-b_{n}\right|^{2}} \text { onde } \lambda_{n}=t_{n}-s_{n},
$$

de (a),

$$
\left|p_{n}-q_{n}\right|^{2}-\left|a_{n}-b_{n}\right|^{2}>\left(n^{2}-1\right)\left|a_{n}-b_{n}\right|^{2}
$$

e, portanto, $\left|\lambda_{n}\right|>\sqrt{n^{2}-1}\left|a_{n}-b_{n}\right|$.

Seja $\alpha_{n}$ o ângulo agudo positivo $\alpha_{n}=A\left(a_{n}-b_{n}, p_{n}-q_{n}\right)$ entre as retas $\left\{\lambda\left(a_{n}-b_{n}\right): \lambda \in \Re\right\}$ e $\left\{\lambda\left(p_{n}-q_{n}\right): \lambda \in \Re\right\} ;$ como $\tan \alpha_{n}=\frac{\left|\lambda_{n}\right|}{\left|a_{n}-b_{n}\right|}>\sqrt{n^{2}-1}$, então $\alpha_{n} \rightarrow \pi / 2$ quando $n \rightarrow+\infty$. Logo $A\left(v, p_{n}-q_{n}\right)$ tende a zero quando $n \rightarrow+\infty$.

Considerando uma subseqüência $v_{n_{k}}=\frac{p_{n_{k}}-q_{n_{k}}}{\left|p_{n_{k}}-q_{n_{k}}\right|} \rightarrow b$, temos $A\left(v, v_{n_{k}}\right) \rightarrow 0$ quando $n_{k} \rightarrow+\infty$, então $b= \pm \frac{v}{|v|}$, pois $|b|=1$.

Assim existe $k_{0}>0$ tal que $w=v_{n_{k_{0}}}>0$ ou $w=v_{n_{k_{0}}}<0$.

Seja $\pi_{w}$ a projeção ortogonal sobre um hiperplano ortogonal a $w$, então $\pi_{w}\left(p_{n_{k_{0}}}-q_{n_{k_{0}}}\right)=0$, $\operatorname{logo} \pi_{w}\left(p_{n_{k_{0}}}\right)=\pi_{w}\left(q_{n_{k_{0}}}\right)$ onde $p_{n_{k_{0}}} \neq q_{n_{k_{0}}}$, o que contradiz o fato de que $\left.\pi_{w}\right|_{L}$ é injetora. Portanto $\left(\left.\pi\right|_{L}\right)^{-1}$ é lipchitziana. Assim, sendo $\left.\pi\right|_{L}$ e $\left(\left.\pi\right|_{L}\right)^{-1}$ contínuas, segue que $\left.\pi\right|_{L}$ è um homeomorfismo.

ii) Definindo $H: \pi(L) \rightarrow E^{n-1}, \quad H(y)=\pi \circ F \circ\left(\left.\pi\right|_{L}\right)^{-1}(y)=\pi(F(x))$ onde $x=\left(\left.\pi\right|_{L}\right)^{-1}(y)$, tem-se que se $x(t)$ é solução de $\left\{\begin{array}{l}\dot{x}=F(x) \\ x(0)=x\end{array}\right.$, então $y(t)=\pi(x(t))$ é solução de $(H)\left\{\begin{array}{l}\dot{y}=H(y) \\ y(0)=y\end{array}\right.$ em $\pi(L)$, pois $\dot{y}=\pi(\dot{x}(t))=\pi(F(x(t)))=H(y(t))$ e $y(0)=$ $\pi(x(0))=\pi(x)=y$.

Dado $q \in \pi(L), \quad q=\left.\pi\right|_{L}(p)$; existe uma vizinhança aberta $V_{p}$ de $p$ em $L$, onde $F$ é lipchitziana e, portanto, como $\pi$ e $\left(\left.\pi\right|_{L}\right)^{-1}$ são lipchitzianas, $H$ é lipchitziana em $\left.\pi\right|_{L}\left(V_{p}\right)$ que é uma vizinhança aberta de $q$ em $\pi(L)$.

Mostraremos agora que $y(t)=\pi(x(t))$ é a única solução de $(H)$ em $\pi(L)$.

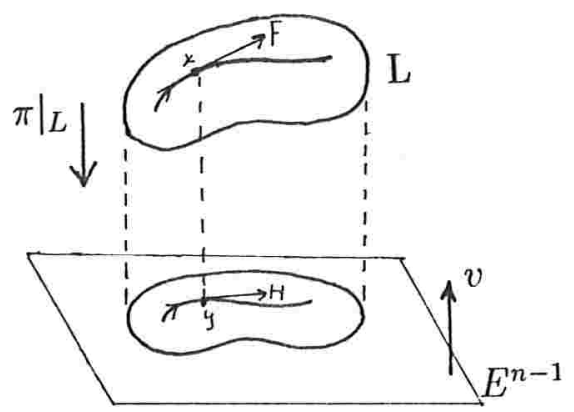


Seja $z \in \pi(L)$ e $V$ uma vizinhança aberta de $z$ em $\pi(L)$ onde $H$ é lipchitziana. Por um resultado de Mcshane (ver apêndice: corolário do Teorema 1) $\left.H\right|_{V}$ admite uma extensão lipchitziana $H_{z}: E^{n-1} \rightarrow E^{n-1}$, logo o problema $\left\{\begin{array}{l}\dot{\xi}=H_{z}(\xi) \\ \xi(0)=z\end{array}\right.$ admite uma única solução em $E^{n-1}$; portanto o problema $\left\{\begin{array}{l}\dot{\xi}=H(\xi) \\ \xi(0)=z\end{array}\right.$ tem uma única solução em $V \subset \pi(L)$. Assim $y(t)=\pi(x(t))$ é a única solução de $(\mathrm{H})$ em $\pi(L)$.

\section{§3. TRAJETÓRIAS COM PONTOS RELACIONADOS}

A seguir apresentamos um teorema que dá uma condição suficiente para a convergência de trajetórias.

PROPOSIÇÃO II.3.1 Se (I) é cooperativo em $W$ p-convexo ; $\overline{O_{+}(x)}=\overline{x([0, \infty))}$ é compacto contido em $W$ e admite dois pontos relacionados, então $\omega(x)=\{p\}$.

Demonstração. Sem perda de generalidade suponhamos que $x(0)<x(T)$ para algum $T>0$. Como $x(t)$ e $y(t)=x(t+T)$ são soluções de $\dot{x}=F(x)$ tais que $x(0)<y(0)$, então pelo teorema de Kamke $x(t) \leq x(t+T)$ para todo $t>0$; $\operatorname{logo} x(k T) \leq x((k+1) T)$ para todo $k \in N$.

Como $\overline{x([0, \infty))}$ é compacto e $x(k T), k \in N$ é monótona, existe $\lim _{k \rightarrow+\infty} x(k T)=p$. Pela continuidade de $\Phi_{T}$ temos que $\lim _{k \rightarrow+\infty} \Phi_{T}(x(k T))=\Phi_{T}(p)$; assim a órbita $\gamma=O_{+}(p)=$ $\left\{\Phi_{t}(p): t \geq 0\right\}$ é fechada, tendo $T$ como um período.

Mostraremos que $\omega(x)=\gamma$ : para isto basta mostrar que se $q \notin \gamma$ então $q \notin \omega(x)$. De fato : seja $d$ a distância de $q$ a $\gamma$ e $\epsilon=\frac{d}{4}>0$, então pela continuidade do fluxo, existe $\delta>0$ tal que para todo $t \in[0, T]$

$$
|y-p|<\delta \Rightarrow|y(t)-p(t)|<\epsilon .
$$

Por outro lado como $\lim _{k \rightarrow+\infty} x(k T)=p$, existe $k_{0} \in N$ tal que para $k \geq k_{0}, k \in N$, $|x(k T)-p|<\delta, \operatorname{logo} d(x(t), \gamma)<\epsilon$ para todo $t \geq k_{0} T$, então $q \notin \omega(x)$. Portanto $\omega(x)=\gamma$ é uma órbita fechada tendo $T$ como um período.

Pela continuidade de $x(t)$ em $T$, de $x(0)<x(T)$ segue-se que existe $\delta>0$ tal que $x(0)<x\left(T^{\prime}\right)$ para todo $T^{\prime} \in[T, T+\delta]$. Assim aplicando o argumento anterior concluímos que $\omega(x)$ é uma órbita fechada tendo os pontos de $[T, T+\delta]$ como períodos, $\operatorname{logo} \omega(x)=\{p\} \cdot \square$

COROLÁRIO II.3.2 Uma órbita fechada não trivial de um sistema (I) cooperativo ou 
competitivo em $W$ p-convexo não tem pontos relacionados.

COROLÁRIO II.3.3 Em $\Re^{3}$, uma órbita fechada não trivial de um sistema (I) cooperativo ou competitivo em $W$ p-convexo não pode ter nós.

Demonstração. Seja $\gamma$ uma órbita peródica não trivial. Suponhamos que $\gamma$ tenha nós, então sua projeção ortogonal sobre qualquer plano não é injetora.

Seja $E^{2} \subset \Re^{2}$ um plano perpendicular a um vetor $v>0$ e $\pi: \Re^{3} \rightarrow E^{2}$ a projeção ortogonal; então $\left.\pi\right|_{\gamma}$ é não injetora, assim existem $x, y \in \gamma, x \neq y$ tais que $\left.\pi\right|_{\gamma}(x)=\left.\pi\right|_{\gamma}(y)$, isto é, $y-x=s v$ onde $s \in \Re, s \neq 0$.

Como $y=x(T)$ para algum $T>0$, então $x$ e $x(T)$ estão relacionados, o que contraria o corolário II.3.2, portanto $\gamma$ não tem nós.

TEOREMA II.3.4. Se (I) é cooperativo em $W$ p-convexo, então o sistema não tem órbita fechada não trivial atratora.

Demonstração. Suponhamos que exista uma órbita fechada $\gamma$ que atrai uma vizinhança $U$ de $\gamma$. Sejam $y \in \gamma$ e $x \in V$ tais que $y<x$; como $y \in \omega(x)$ então existe $T>0$ tal que $x(T)<x$, pela proposição II.3.1, $\omega(x)=\{p\}=\gamma$, tendo em vista que $\overline{O_{+}(x)} \subset W$ é compacto.

OBSERVAÇÃO II.3.5 Deste teorema segue-se que um sistema (I) competitivo em $W$ p-convexo não admite órbita fechada não trivial repulsora.

OBSERVAÇÃO II.3.6 Se $\omega(x) \neq \emptyset$ é compacto, então $\overline{O_{+}(x)}=O_{+}(x) \cup \omega(x)$ é compacto. Basta mostrar que $O_{+}(x)$ é limitado para concluir que $\overline{O_{+}(x)}$ é compacto. Como $\omega(x)$ é limitado, existe uma bola aberta $B$ de centro $O=(o, \ldots, o)$ e raio $R>0$ tal que $\omega(x) \subset B$. Seja $p \in \omega(x), p=\lim _{s_{k} \rightarrow+\infty} x\left(s_{k}\right)$. Para $s_{k}$ suficientemente grande $x\left(s_{k}\right) \in B$. Se $O_{+}(x)$ não fosse limitada, existiriam tempos $s_{k}<r_{k}<t_{k}$ tais que $\left|x\left(t_{k}\right)\right|>2 R$ e $R \leq\left|x\left(r_{k}\right)\right| \leq 2 R$. Como $x\left(r_{k}\right)$ admite uma subseqüência convergente para $q \operatorname{com} R \leq|q| \leq 2 R$ e $q \in \omega(x)$ chegamos a uma contradição. Assim $O_{+}(x)$ é limitada.

Seja $p \in \overline{O_{+}(x)}-O_{+}(x)$ e $x\left(t_{n}\right) \rightarrow p$. Pela compacidade de $\overline{O_{+}(x)}, x(t)$ está definida para todo $t \geq 0$ e portanto, podemos afirmar que $t_{n} \rightarrow+\infty$. Logo $p \in \omega(x)$. Segue-se assim que $\overline{O_{+}(x)}=O_{+}(x) \cup \omega(x)$.

Como aplicação da proposição II.3.1 apresentamos um teorema análogo ao teorema A para campos cooperativos. 
TEOREMA II.3.7 Seja (I) um sistema cooperativo em $W$ p-convexo, $K=\overline{O_{+}(x)} \subset W$ compacto. Se $x(t)$ não converge para um equilíbrio, então $K$ é compressível ao longo de qualquer vetor positivo.

Demonstração. Dado $v \in \Re^{n}, v>0$, consideremos $E^{n-1}$ um hiperplano ortogonal a $v$ e $\pi_{v}: \Re \rightarrow E^{n-1}$ a projeção ortogonal.

Mostraremos que $\left.\pi_{v}\right|_{K}$ é um homeomorfismo com $\left(\left.\pi_{v}\right|_{K}\right)^{-1}$ lipchitziana.

i) Suponhamos que $\left.\pi_{v}\right|_{K}$ não seja injetora, então existem $p, q \in K, p \neq q$ tais que $\left.\pi_{v}\right|_{K}(p)=\left.\pi_{v}\right|_{K}(q)$; então $p$ e $q$ estão relacionados, por exemplo $p<q$. Como $K=\overline{O_{+}(x)}=$ $O_{+}(x) \cup \omega(x)$, se $p, q \in O_{+}(x)$ então existem $t_{0}, t_{1} \geq 0$ tais que $x\left(t_{0}\right)=p<q=x\left(t_{1}\right)$ e se $p \in \omega(x), q=x(\bar{t})$, como $p=\lim _{t_{k} \rightarrow+\infty} x\left(t_{k}\right)$ existe $t_{k}>0$ tal que $t_{k}>\bar{t}$ e $x\left(t_{k}\right)<x(\bar{t})$.

ii) Supondo que $\left(\left.\pi_{v}\right|_{K}\right)^{-1}$ não seja lipchitziana, seguindo a mesma demonstração feita no teorema A concluímos que existe um vetor $w>0$ tal que $\left.\pi_{w}\right|_{K}$ não é injetora, então por i) existem dois pontos relacionados na $O_{+}(x)$.

Assim, se $\left.\pi_{v}\right|_{K}$ não é injetora ou $\left(\left.\pi_{v}\right|_{K}\right)^{-1}$ não é lipchitziana, existem dois pontos relacionados na $O_{+}(x)$; logo pela proposição II.3.1, $x(t)$ converge para um ponto de equilíbrio, o que contradiz a hipótese. A demonstração prossegue como no teorema A.

\section{§. CONJUNTOS LIMITE. EM $\Re^{3}$}

Veremos a seguir um resultado análogo ao teorema de Poincaré - Bendixson para campos cooperativos ou competitivos em $\Re^{3}$.

TEOREMA $B$ Seja $L \neq \emptyset$ um conjunto limite compacto sem pontos de equilíbrio de um campo $F$ cooperativo ou competitivo num aberto p-convexo $W \subset \Re^{3}$. Então $L$ é um cilindro de órbitas fechadas. Além disso, se $F$ é cooperativo e $L$ é $\omega$-limite, então $L$ é uma única órbita periódica.

A segunda parte do teorema foi melhorada por Smith [19] que, colocando uma hipótese adicional no campo cooperativo, conclue que mesmo no caso em que $L$ é um conjunto $\alpha$-limite, ele é uma única órbita fechada.

Comentaremos informalmente tal resultado no próximo capítulo após a proposição III.3.4.

Demonstração do teorema B. Sejam $v>0, E^{2}$ um plano ortogonal ao vetor $v$ e $\pi: \Re^{3} \rightarrow E^{2}$ a projeção ortogonal. Pelo teorema A, $L$ é compressível ao longo de $v$, isto é, $\left.\pi\right|_{L}$ é um homeomorfismo com $\left(\left.\pi\right|_{L}\right)^{-1}$ lipchitziana que leva as trajetórias do campo $F$ em $L$ nas trajetórias do campo $H=\pi \circ F \circ\left(\left.\pi\right|_{L}\right)^{-1}: \pi(L) \rightarrow E^{2}$ que é localmente lipchitziano em $\pi(L)$. 
Demonstraremos que $H$ é lipchitziana em $\pi(L)$ e, portanto, por um resultado de Mcshane (ver apêndice : corolário do teorema 1) admite uma extensão lipchitziana $\tilde{H}: E^{2} \rightarrow E^{2}$.

Como $\mathrm{H}$ é localmente lipchitziana em $\pi(L)$, para cada $z \in \pi(L)$ existe uma vizinhança aberta $V_{z}$ de $z$ em $E^{2}$ tal que $H: V_{z} \cap \pi(L) \rightarrow E^{2}$ é lipchitziana, sendo $M_{z}$ a constante de Lipchitz. Da compacidade de $K=\pi(L)$ tem-se $K \subset \bigcup_{i=1}^{n} V_{z_{i}}$. Seja

$$
D=(K \times K)-\bigcup_{i=1}^{n}\left(V_{z_{i}} \times V_{z_{i}}\right)
$$

e $g: D \rightarrow \Re$ uma função definida por

$$
g(u, v)=\frac{|H(u)-H(v)|}{|u-v|} .
$$

Como $g$ é contínua no compacto $D$, então existe $M>0$ tal que $|g(u, v)| \leq M$ em $D$, isto é, $|H(u)-H(v)| \leq M|u-v|$ para todo $(u, v) \in D$. Para $N=\max \left\{M, M_{z_{i}}, i=1,2, \ldots, n\right\}$ temos $|H(u)-H(v)| \leq M_{z_{i}}|u-v| \leq N|u-v|$ se $(u, v) \in V_{z_{i}} \times V_{z_{i}}$ para $i=1,2, \ldots, n$ e $|H(u)-H(v)| \leq M|u-v| \leq N|u-v|$ se $(u, v) \in D$; isto é, $H$ é lipchitziana em $\pi(L) \subset E^{2}$.

Como $\pi(L)$ é compacto e invariante pelo fluxo de $H$, então os conjuntos limites de soluções de $H$ são descritos pelo teorema das Poincaré - Bendixson [6] aplicado ao sistema definido por $\tilde{H}$ em $E^{2}$ : logo são órbitas fechadas não triviais, já que $\pi(L)$ não contém equilíbrios (ver demonstração de ii do teorema A). Donde, concluiremos que $\pi(L)$ é união de órbitas fechadas não triviais mostrando que não existe solução regular em $\pi(L)$.

Suponhamos que exista $z \in \pi(L)$ tal que $z(t)$ seja uma solução regular de $H$. Seja $\gamma=\omega(z)$, então pelo argumento acima $\gamma$ é uma órbita fechada; seja $T>0$ o período de $\gamma$.

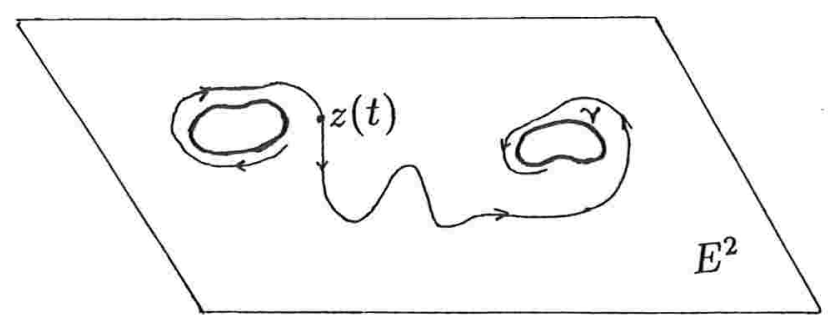


Sem perda de generalidade suporemos $L=\omega(x)$ para algum $x \in W$ (pois se $L=\alpha(x)$ basta trabalhar com o campo $-F)$.

Seja $\Phi_{t}$ o fluxo de $F$. Mostraremos que existe um aberto $U$ de $L, \emptyset \neq U \mp L$ tal que $\Phi_{T}(\bar{U}) \subset U$, para algum $T>0$, o que contraria o seguinte resultado: "Se $\omega(x)$ é compacto e contém alguma órbita regular, então não existe subconjunto $U$ próprio de $\omega(x), U \neq \emptyset, U$ aberto de $\omega(x)$ tal que $\Phi_{T}(\bar{U}) \subset U$ para algum $T>0$ " (ver teorema 2 do apêndice).

Sejam $\psi_{t}$ o fluxo de $\tilde{H}, A$ a componente de $E^{2}-\gamma$ que contém $z$ e $B$ a outra componente. Como $\gamma$ é atrator para o fluxo restrito a $\bar{A}$, existe uma vizinhança compacta $N$ de $\gamma$ em $\bar{A}$ tal que $\psi_{T}(N-\gamma) \subset \operatorname{int} N$.

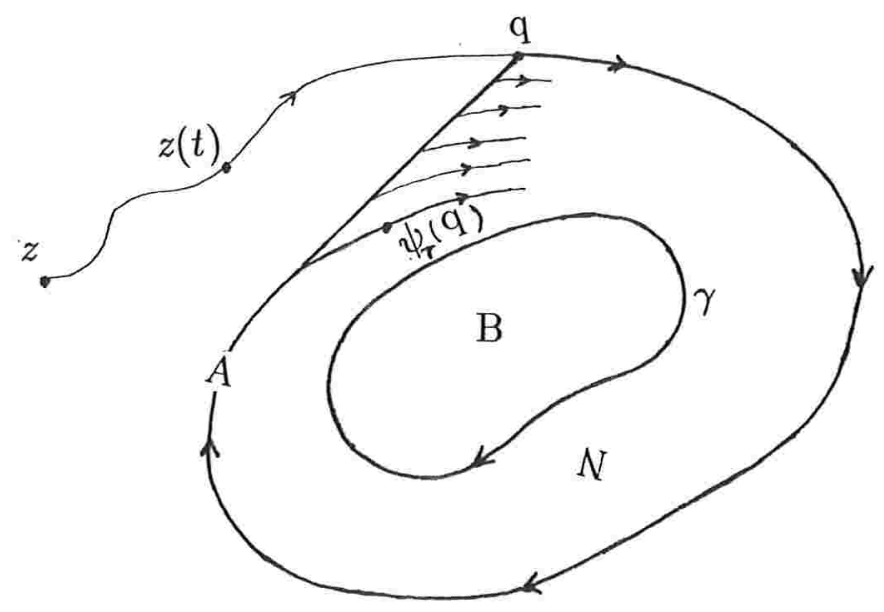

Tomamos $U=\left(\left.\pi\right|_{L}\right)^{-1}(R)$ onde $R=\pi(L) \cap(\stackrel{\circ}{(B \cup N}) \neq \emptyset$

Mostraremos que $\Phi_{T}(\bar{U}) \subset U:$ seja $w=\Phi_{T}(v), \operatorname{com} v \in \bar{U}, \operatorname{como} \bar{U}=\overline{\left(\left.\pi\right|_{L}\right)^{-1}(R)}=$ $\left(\left.\pi\right|_{L}\right)^{-1}(\bar{R})$, então, $v=\left(\left.\pi\right|_{L}\right)^{-1}(y)$ com $y \in \bar{R}$. Assim $w=\Phi_{T}\left(\left(\left.\pi\right|_{L}\right)^{-1}(y)\right)=\left(\left.\pi\right|_{L}\right)^{-1}\left(\psi_{T}(y)\right)$ e como $\psi_{T}(\bar{R}) \subset R$, então $w \in\left(\left.\pi\right|_{L}\right)^{-1}(R)=U$.

Assim, mostramos que $\pi(L)$ é união de órbitas fechadas não triviais; como $\pi(L)$ é conexo (pois $L$ é conexo), então $\pi(L)$ é um cilindro de órbitas fechadas em $E^{2}$, portanto por intermédio do homeomorfismo $\left(\left.\pi\right|_{L}\right)^{-1}, L$ é um cilindro de órbitas periódicas não triviais do campo $F$.

Mostraremos agora que se $F$ é cooperativo e $L$ é um conjunto $\omega$-limite, então $L$ é uma órbita fechada. 
Suponhamos que $L$ não fosse uma única órbita periódica, neste caso $O_{+}(x) \cap L=\emptyset \mathrm{e}$ $\pi(L)$ contém algum disco bidimensional $D$ com centro $\pi(p)$ onde $p \in L$. Como $L=\omega(x)$, $p=\lim _{k \rightarrow+\infty} x\left(t_{k}\right)$, então existe $\bar{t}>0$ tal que $\pi(x(\bar{t})) \in \operatorname{int} D \subset \pi(L)$. Seja $q \in L$ tal que $\pi(q)=\pi(x(\bar{t}))$ e portanto, como $x(\bar{t}) \neq q$, então $x(\bar{t})>q($ ou $x(\bar{t})<q)$, logo existe $t>\bar{t}>0$ tal que $x(\bar{t})>x(t)$ (ou $x(\bar{t})<x(t))$ e a contradição segue da proposição II.3.1. 口

Usando o teorema II.3.7 pode-se demonstrar os seguintes resultados para conjuntos $\omega$-limites de sistemas cooperativos.

PROPOSIÇÃO II.4.1 Sendo (I) cooperativo num aberto p-convexo $W \subset \Re^{3}$ e $L$ um conjunto $\omega$-limite compacto, tem-se:

i) se $L$ contém uma órbita fechada não trivial $\gamma$, então $L=\gamma$;

ii) se $L$ não contém órbita fechada e contém somente um número finito de pontos de equilíbrio $p_{1}, \ldots, p_{m}, m \geq 1$, mas $x(t)$ não converge, então $p_{1}, \ldots, p_{m}$ determina um ciclo de equilíbrio contido em $L$ (os pontos de equilíbrio $p_{1}, \ldots, p_{m}, m \geq 1$ determinam um ciclo de equilíbrio em $L$ se para cada $i=1, \ldots, m$ existe uma solução regular $y_{i}(t) \mathrm{em} L$ cujo $\alpha$ - limite é $p_{i}$ e cujo $\omega$-limite é $\left.p_{i+1} \operatorname{com} p_{m+1}=p_{1}\right)$.

\section{Demonstração da proposição II.4.1}

i) Suponhamos que $L=\omega(x)$ contenha uma órbita fechada $\gamma$ não trivial. Como $L$ é compacto, segue que $K=\overline{O_{+}(x)} \subset W$ é compacto e como $x(t)$ não é convergente, então pelo teorema II.3.7 $K$ é compressível ao longo de cada vetor positivo.

Com as mesmas definições usadas na demonstração do teorema II.3.7 e teorema $\mathrm{B} ;$ seja $z=\pi(x)$, logo $\psi_{t}(z)$ tem como conjunto limite $\omega(z)=\pi(L) \subset \pi(K), \pi(L)$ contém a órbita fechada não trivial $\pi(\gamma)=\gamma^{\prime}$, logo pelo teorema de Poincaré - Bendixson aplicado ao campo $\tilde{H}: E^{2} \rightarrow E^{2}$, obtido analogamente ao do teorema $\mathrm{B}$, tem-se que $\left.\pi\right|_{K}(L)=\left.\pi\right|_{K}(\gamma)$ e sendo $\left.\pi\right|_{K}$ injetora conclui-se que $L=\gamma$.

ii) Se $L$ não contém uma órbita fechada e contém somente um número finito de pontos de equilíbrio $p_{1}, \ldots, p_{m}, m \geq 1$, então $\omega(z)=\left.\pi\right|_{K}(L)$ contém um número finito de pontos de equilíbrio $\left.\pi\right|_{K}\left(p_{1}\right), \ldots,\left.\pi\right|_{K}\left(p_{m}\right), m \geqslant 1$ e pelo teorema de Poincarè - Bendixson aplicado ao campo $\tilde{H}$, tem-se que $\pi(L)$ contém um ciclo determinado por $\left.\pi\right|_{K}\left(p_{1}\right), \ldots,\left.\pi\right|_{K}\left(p_{m}\right)$; portanto $L$ contém um ciclo de equilíbrio determinado por $p_{1}, \ldots, p_{m} \cdot \square$ 
COROLÁRIO II.4.3 Seja (I) um sistema cooperativo, definido num aberto p-convexo $W \subset \Re^{3}$. Se $\emptyset \neq L \subset W$ é um conjunto $\omega$-limite compacto que não contém um ciclo de equilíbrio, então $L$ é uma órbita fechada não trivial ou $L$ é um ponto de equilíbrio. 


\section{CAPÍTULO III. SISTEMAS COOPERATIVOS IRREDUTÍVEIS}

Neste capítulo estudaremos essencialmente o comportamento dos conjuntos $\omega$ limite dos sistemas cooperativos cujas matrizes jacobianas são irredutíveis.

Como resultado central veremos que quase todo ponto com trajetória positiva de fecho compacto, tem $\omega$-limite contido no conjunto dos pontos de equilíbrio.

Continuaremos neste capítulo considerando sistemas

$$
\dot{x}=F(x)
$$

com $F: W \rightarrow \Re^{n}$ de classe $C^{1}$ num aberto $W \subset \Re^{n}$.

\section{§1. FLUXOS COM DERIVADA POSITIVA.}

DEFINIÇÃO III.1.1 Uma matriz real $A=\left[a_{i j}\right]$ de ordem $n \times n$ é chamada irredutível se não existe um subconjunto próprio $\left\{k_{1}, k_{2}, \ldots, k_{r}\right\}$ de $\{1,2, \ldots, n\}$ tal que $\left.<A e_{k_{1}}, \ldots, A e_{k_{r}}\right\rangle$ $\subset<e_{k_{1}}, \ldots, e_{k_{r}}>$, onde $e_{k_{i}}$ são os vetores da base canônica de $\Re^{n} \mathrm{e}<>$ denota o subespaço gerado pelos vetores considerados.

\section{EXEMPLOS :}

1) Matrizes de Jacobi

$$
\left(\begin{array}{cccccc}
a_{1} & c_{1} & 0 & \cdot & \cdot & 0 \\
b_{2} & a_{2} & \cdot & \cdot & \cdot & \cdot \\
0 & \cdot & \cdot & \cdot & \cdot & \cdot \\
\cdot & \cdot & \cdot & \cdot & \cdot & 0 \\
0 & \cdot & \cdot & \cdot & \cdot & c_{n-1} \\
0 & 0 & \cdot & 0 & b_{n} & a_{n}
\end{array}\right)
$$

com $b_{i}>0, c_{i}>0$ para todo $i$.

2) Matrizes cíclicas 


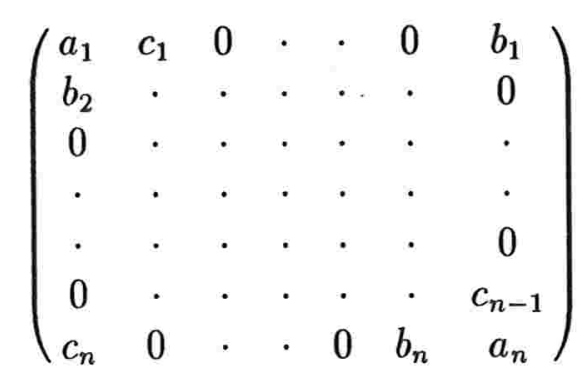

$\operatorname{com} b_{i}, c_{i} \geq 0, i=1,2, \ldots, n$ e $\prod_{i=1}^{n} b_{i}+\prod_{i=1}^{n} c_{i}>0$.

(ver exemplo 4 do capítulo I, §1.)

OBSERVAÇÃO III.1.2 A definição de matriz irredutível também pode ser formulada das seguintes maneiras equivalentes:

1) A matriz real $A=\left[a_{i j}\right]_{n \times n}$ é irredutível se para todo subconjunto próprio $J$ de $I=$ $\{1,2, \ldots, n\}$ existem $i \in J$ e $j \in I-J$ tais que $a_{i j} \neq 0$.

2) A matriz real $A=\left[a_{i j}\right]_{n \times n}$ é irredutível se não existe uma permutação da base canônica onde $A$ se escreve na forma

$$
\tilde{A}=\left(\begin{array}{ll}
B & C \\
0 & D
\end{array}\right)
$$

onde $B$ e $D$ são matrizes quadradas não necessariamente da mesma ordem.

DEFINIÇÃO III.1.3 O sistema (I) é chamado irredutível se a matriz $D F(x)$ é irredutível para cada $x \in W$.

EXEMPLOS : No capítulo I, $\S 1$ : o exemplo 2) no int. $\Re_{+}^{3}$ e o exemplo 4).

Vimos na proposição I.2.3 que se o sistema (I) é cooperativo, então $D \Phi_{t}(x) \geq 0$ para todo $x \in W$ e $t \geqslant 0$ do intervalo maximal de $\Phi_{t}(x)$. Agora, com a condição adicional de irredutibilidade, mostraremos que $D \Phi_{t}(x)>0$ para todo $x \in W$ e $t>0$.

PROPOSIÇÃO III.1.4 Se (I) é cooperativo irredutível, então seu fluxo $\left\{\Phi_{t}\right\}$ tem derivadas estritamente positivas; isto é, para $x \in W$ e $t>0$ no intervalo maximal de $\Phi_{t}(x)$, tem-se $D \Phi_{t}(x)>0$.

Demonstração. Pela proposição I.2.3, já sabemos que $D \Phi_{t}(x) \geq 0$. Seja $M(t)=D \Phi_{t}(x)$ a solução do problema

$$
\left\{\begin{array}{l}
\dot{M}=A(t) M \\
M(0)=\text { Id }
\end{array}\right.
$$

onde $A(t)=\left[A_{i j}(t)\right]_{n \times n}=D F\left(\Phi_{t}(x)\right)$, Id. matriz identidade. 
Para cada $t^{\prime}>0$ no intervalo maximal de $\Phi_{t}(x)$, mostraremos que existe $t^{*}$ com $0<t^{*}<t^{\prime}$ tal que $M\left(t^{*}\right)>0$; logo pelo teorema de Kamke teremos que $M\left(t^{\prime}\right)>0$.

Como $F$ é cooperativo irredutível, então a matriz $A(t)$ é irredutível e $A_{i j}(t) \geq 0$ para todo $i \neq j$. Assim, existe $r \geq 2$ tal que $A_{r 1}(0)>0$ e, portanto, $\dot{M}_{r 1}(0)>0$, já que

$$
\dot{M}_{r 1}(0)=\sum_{s=2}^{n} A_{r s}(0) M_{s 1}(0)+A_{r 1}(0) M_{11}(0)
$$

Logo existe $\delta_{1}, 0<\delta_{1}<t^{\prime}$ tal que $M_{r 1}(t)>0$ para todo $t \in\left(0, \delta_{1}\right)$; diminuindo eventualmente $\delta_{1}$, podemos admitir que para todo $i=1, \ldots, n, M_{i i}(t)>0$ com $t \in\left(0, \delta_{1}\right)$, já que $M_{i i}(0)=1>0$. Assim, existe $\left(a_{1}, b_{1}\right) \subseteq\left(0, \delta_{1}\right)$ onde pelo menos $(n+1)$ elementos da matriz $M(t)$ são positivos.

Se existe algum $t \in\left(a_{1}, b_{1}\right)$ tal que $M(t)>0$, então tomamos $t^{*}=t$. Em caso contrário dado $t_{1} \in\left(a_{1}, b_{1}\right)$ existem $i, j$ tais que $M_{i j}\left(t_{1}\right)=0$ e, então, fixado $j$ consideramos

$$
J=\left\{s \mid M_{s j}\left(t_{1}\right)=0\right\}
$$

que é um subconjunto próprio de $\{1,2, \ldots, n\}=I$. Como a matriz $A\left(t_{1}\right)$ é irredutível, existem $s \in J$ e $k \in I-J$ tais que $A_{s k}\left(t_{1}\right) \neq 0$. Assim de $A_{s k}\left(t_{1}\right)>0, M_{k j}\left(t_{1}\right)>0$ e de

$$
\dot{M}_{s j}\left(t_{1}\right)=A_{s k}\left(t_{1}\right) M_{k j}\left(t_{1}\right)+\sum_{r \neq k} A_{s r}\left(t_{1}\right) M_{r j}\left(t_{1}\right)
$$

tem-se que $\dot{M}_{s j}\left(t_{1}\right)>0$. Portanto existe $\sigma_{1} \in\left(t_{1}, \delta_{1}\right)$ tal que $M_{s j}(t)>0$ para todo $t \in\left(t_{1}, \sigma_{1}\right)$ e, então, pelo menos $(n+2)$ elementos da matriz $M(t)$ são positivos em $\left(t_{1}, \sigma_{1}\right) \underset{\leftarrow}{\tau}\left(a_{1}, b_{1}\right)$. Seja $\left(a_{2}, b_{2}\right)=\left(t_{1}, \sigma_{1}\right)$, se existe algum $t \in\left(a_{2}, b_{2}\right)$ tal que $M(t)>0$, então tomamos $t^{*}=t$. Caso contrário, repete-se o processo anterior e assim encontramos intervalos $\left(a_{3}, b_{3}\right)=\left(t_{2}, \sigma_{2}\right) \subsetneq\left(a_{2}, b_{2}\right) \subsetneq\left(a_{1}, b_{1}\right)$ tal que pelo menos $(n+3)$ elementos da matriz $M(t)$ são positivos em $\left(t_{2}, \sigma_{2}\right)$, portanto em $\left(a_{3}, b_{3}\right)$.

$\mathrm{E}$ assim, sucessivamente, continuamos o processo anterior um número finito de vezes e, portanto, conseguimos $t^{*}>0$ tal que $M_{i j}\left(t^{*}\right)>0$ para todo $i, j$, ou seja, $M\left(t^{*}\right)>$ 0. ㅁ

Conforme observamos após o teorema B, Smith [19] demonstrou o seguinte teorema: "Se $L \neq \emptyset$ é um conjunto $\alpha$-limite compacto sem pontos de equilíbrio de um sistema (I) cooperativo irredutível de classe $C^{2}$ num aberto p-convexo de $\Re^{3}$, então $L$ é uma órbita fechada". Em seguida comentaremos informalmente a demonstração que usa o seguinte resultado:

TEOREMA DE PERRON [5] "Uma matriz $A>0$ admite um autovalor real $\rho>0$ simples tal que $\rho>|\lambda|$ para qualquer outro autovalor $\lambda$ de $A$. Além disso $\rho$ admite um autovetor $v>0 . "$

Pelo teorema B, $L$ é um cilindro de órbitas fechadas. Suponhamos que não fosse uma única órbita. 
Sejam $\gamma$ uma órbita fechada contida em $L$ de período $T>0$ e $q \in \gamma$.

Consideremos a aplicação $x \mapsto \Phi_{T}(x)$ com ponto fixo $q$. Como o sistema é cooperativo irredutível, então, $D \Phi_{T}(q)>0$, portanto pelo teorema de Perron a matriz $D \Phi_{T}(q)$ tem um autovalor $\rho \in \Re, \rho>0$ tal que $\rho>|\lambda|$ para qualquer outro autovalor $\lambda$, com autovetor $v>0: D \Phi_{T}(q) v=\rho v$.

Como $D \Phi_{T}(q) F(q)=F\left(\Phi_{T}(q)\right)=F(q)$ e pela proposição II.1.5, $F(q) \ngtr 0$ e $F(q) \nless 0$, então, $\rho>1$.

Sejam $\Sigma$ uma seção plana transversal ao fluxo em $q$ e $P$ a transformação de Poincaré definida numa vizinhança $\Sigma_{0} \subset \Sigma$ de $q$ com valores em $\Sigma$. Então $P$ tem em $q$ uma variedade unidimensional $\Gamma$ instável correspondente ao autovalor $\rho>1$ de $D P(q)$.

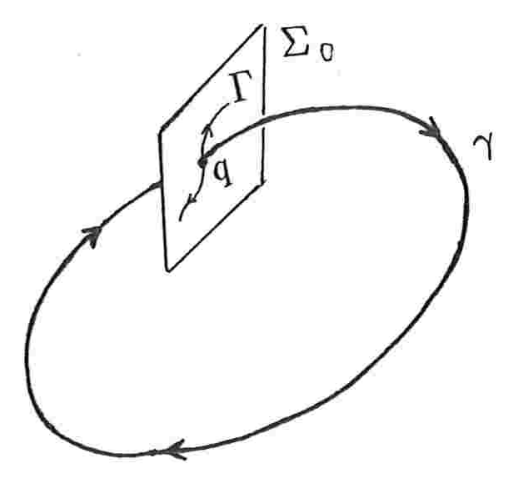

Seja $\sigma$ a intersecção de $L$ com $\Sigma_{0}$ formada por pontos fixos de $P$.

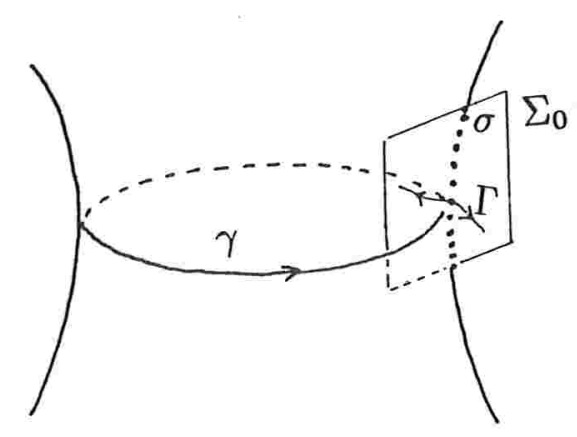


Como $D P(q)$ tem 1 como autovalor pois qualquer vizinhança de $q$ em $\Sigma$ tem pontos fixos de $P$, então $P$ tem uma variedade central unidimensional de classe $C^{1}$ em $q$, que coincide com $\sigma$ (ver teorema da variedade central em [14]).

As variedades instáveis $\Gamma^{\prime}$ de $P$ correspondentes aos pontos $q^{\prime} \in \sigma$ são disjuntas duas a duas e constituem localmente em $q$ uma fibração contínua de $\Sigma_{0}$.

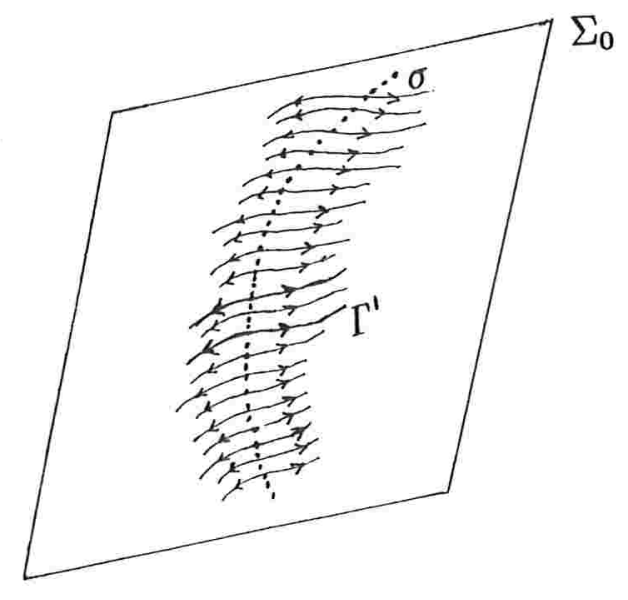

Aplicando o fluxo obtemos uma fibração de uma vizinhança aberta $V$ da órbita $\gamma$, com fibras bidimensionais de instabilidade.

Suponhamos que $L$ seja $\alpha$-limite de algum ponto $x \in W$, então, para algum $t_{0}<<0, x\left(t_{0}\right) \in V$ e, portanto, cai sobre uma fibra de instabilidade. Daí segue que $x(t)$ tende somente a uma órbita fechada $\gamma^{\prime} \subset L$, logo $L=\gamma^{\prime}$ o que contraria a hipótese assumida para $L$. 口

DEFINIÇÃO III.1.5 Seja $U \subset \Re^{n}$, dizemos que uma aplicação $f: U \rightarrow \Re^{n}$ é monótona em $U$ (fortemente monótona) se para $x, y \in U$ tais que $x \leq y$ tem-se que $f(x) \leq f(y)$ $(x \leq y$ e $x \neq y$ implica $f(x)<f(y))$.

PROPOSIÇÃO III.1.6 Seja $W$ um aberto de $\Re^{n}$ p-convexo e $F: W \rightarrow \Re^{n}$ um campo vetorial de classe $C^{1}$. Se $F$ é cooperativo (cooperativo irredutível) então, seu fluxo $\left\{\Phi_{t}\right\}$ é monótono (fortemente monótono). 
Demonstração. Sejam $x, y \in W \operatorname{com} x \leq y$, como $W$ é p-convexo $g(s)=x+s(y-x) \in W$ para todo $s \in[0,1]$. Temos:

$$
\Phi_{t}(y)-\Phi_{t}(x)=\int_{0}^{1} D \Phi_{t}(g(s)) \cdot(y-x) d s
$$

De fato:

$$
\begin{aligned}
& \left(\Phi_{t} \circ g\right)^{\prime}(s)=D \Phi_{t}(g(s)) \cdot(y-x), \text { logo } \\
& \Phi_{t}(y)-\Phi_{t}(x)=\left(\Phi_{t} \circ g\right)(1)-\left(\Phi_{t} \circ g\right)(0)=\int_{0}^{1}\left(\Phi_{t} \circ g\right)^{\prime}(s) d s=\int_{0}^{1} D \Phi_{t}(g(s)) \cdot(y-x) d s .
\end{aligned}
$$

Como $F$ é um campo cooperativo em $W$, então, pela proposição I.2.3, $D \Phi_{t}(x) \geq 0$ para todo $x \in W, t \geq 0, t \in I$; logo de $(*), \Phi_{t}(y)-\Phi_{t}(x) \geq 0$, portanto, $\Phi_{t}$ é monótona em $W$ para todo $t \geq 0, t \in I$.

Se F é um campo cooperativo irredutível em $W$, então pela proposição III.1.4 $D \Phi_{t}(x)>0$ para todo $x \in W, t>0, t \in I$; assim para $x \leq y, x \neq y$ tem-se $h(s)=$ $D \Phi_{t}(g(s)) \cdot(y-x)>0$ para todo $s \in[0,1]$. Como $h$ é contínua no intervalo [0,1], então tem valor mínimo $h\left(s_{0}\right)=\alpha>0$; assim

$$
\int_{0}^{1} h(s) d s \geq \alpha \int_{0}^{1} d s=\alpha>0
$$

então, de $(*)$ segue que $\Phi_{t}(y)>\Phi_{t}(x)$, portanto $\Phi_{t}$ é fortemente monótona em $V$. 口

\section{§2. RELAÇÃO ENTRE OS CONJUNTOS $\omega$-LIMITE DE PONTOS PARCI- ALMENTE ORDENADOS}

De agora em diante, neste capítulo, consideraremos o sistema (I) onde $F$ é um campo de classe $C^{1}$ num aberto $W$ p-convexo de $\Re^{n}$, cooperativo irredutível; portanto com fluxo $\left\{\Phi_{t}\right\}$ fortemente monótono em $W$. Indicaremos $x(t)=\Phi_{t}(x)=x . t$ e denotaremos com $E$ o conjunto dos pontos de equilíbrio.

Enunciamos em seguida um teorema importante para tais sistemas que estabelece uma relação entre os conjuntos $\omega$-limite de pontos parcialmente ordenados.

TEOREMA III.2.1 Sejam $x, y \in W$ tais que $x \leq y, x \neq y \operatorname{com} \overline{O_{+}(x)} \cup \overline{O_{+}(y)} \subset W$ e $\overline{O_{+}(x)}, \overline{O_{+}(y)}$ compactos. Então tem-se uma das seguintes condições:

i) $\omega(x)=\omega(y) \subset E$; ou

ii) $\omega(x)<\omega(y)$ 
Com o propósito de demonstrar este teorema, veremos antes outros resultados.

DEFINIÇÃO III.2.2 Sejam $x$ e $y$ pontos de $W$. Um ponto $p \in W$ é chamado $\omega$-colimite de $x$ e $y$ se existe alguma seqüência $t_{k} \rightarrow+\infty$ tal que $p=\lim _{k \rightarrow+\infty} x\left(t_{k}\right)=\lim _{k \rightarrow+\infty} y\left(t_{k}\right)$.

PROPOSIÇÃO III.2.3 (princípio do colimite)

Sejam $x, y \in W, x \leq y, x \neq y$. Se $p \in W$ é um ponto $\omega$-colimite de $x$ e $y$, então $p$ é um ponto de equilíbrio.

Demonstração. Como o fluxo é fortemente monótono segue-se que $x(1)<y(1)$; logo suporemos que $x<y$. Seja $\tau>0$ tal que $x(s)<y, x<y(s)$ para todo $s \in[0, \tau]$

e que além disso $p$ esteja definido em $[0, \tau]$. Pela monotonicidade de $\Phi_{t_{k}}$ temos:

$\Phi_{t_{k}}(x(s))<\Phi_{t_{k}}(y)$ e $\Phi_{t_{k}}(x)<\Phi_{t_{k}}(y(s))$ para $s \in[0, \tau]$ ou equivalentemente

$\Phi_{s}\left(x\left(t_{k}\right)\right)<y\left(t_{k}\right)$ e $x\left(t_{k}\right)<\Phi_{s}\left(y\left(t_{k}\right)\right)$ para $s \in[0, \tau]$

assim tomando limite quando $k \rightarrow+\infty$ temos $\Phi_{s}(p) \leq p$ e $p \leq \Phi_{s}(p), \operatorname{logo} \Phi_{s}(p)=p$ para todo $s \in[0, \tau]$, isto é $p$ é um ponto de equilíbrio. $\square$

\section{COROLÁRIO III.2.4 (princípio da intersecção)}

Sejam $x, y \in W, x \leq y, x \neq y \operatorname{com} \overline{O_{+}(x)}, \overline{O_{+}(y)}$ compactos contidos em $W$, não vazios. Então $\omega(x) \cap \omega(y) \subset E$.

Demonstração. Se $p \in \omega(x) \bigcap \omega(y)$, então existe uma seqüência $t_{k} \rightarrow+\infty$ tal que $p=$ $\lim _{k \rightarrow+\infty} x\left(t_{k}\right)$. A seqüência $\left\{y\left(t_{k}\right)\right\}$ está contida no compacto $\overline{O_{+}(y)}=O_{+}(y) \cup \omega(y) \subset W$, portanto tem uma subseqüência convergente $y\left(t_{k_{l}}\right) \rightarrow q$. Como $x\left(t_{k_{l}}\right)<y\left(t_{k_{l}}\right)$ pela monotonicidade do fluxo; então tomando limite tem-se que $p \leq q$.

Mostraremos que $p=q$ e assim pela proposição III.2.3, concluiremos que $p \in E$. Suponhamos que $p \neq q$, como $x\left(t_{k_{l}}-T\right)<y\left(t_{k_{l}}-T\right)$ para $t_{k_{l}}>T>0$, temos

$p(-T)=\lim _{t_{k_{l}} \rightarrow+\infty} x\left(t_{k_{l}}-T\right) \leq \lim _{t_{k_{l}} \rightarrow+\infty} y\left(t_{k_{l}}-T\right)=q(-T)$

assim, $\Phi_{T}(p(-T))<\Phi_{T}(q(-T))$, ou seja, $p<q$ o que é falso pela proposição II.1.5, já que $p, q \in \omega(y)$. व 
OBSERVAÇÃO III.2.5 A proposição III.2.3 também é válida para sistemas (I) cooperativos não irredutíveis, definidos num aberto p-convexo de $\Re^{n}$ desde que se considere $x<y$.

DEFINIÇÃO III.2.6 Um conjunto compacto $K$ é invariante, pelo fluxo $\Phi_{t}$ se $\Phi_{t}(K)=K$ para todo $t \in \Re$.

Por exemplo os conjuntos $\omega$-limite são invariantes.

Notação: Dados dois conjuntos não vazios $A$ e $B$, se para todo $a \in A$ e $b \in B$ tem-se que $a<b \quad(a \leq b)$, então escreveremos $A<B \quad(A \leq B)$.

PROPOSIÇÃO III.2.7 (princípio de absorção)

Seja $K \subset W$ compacto invariante pelo fluxo $\Phi_{t}$ tal que $K<c(K>c)$ onde $c \in \omega(z)$ e $z \in W$, então $K<\omega(z) \quad(K>\omega(z))$.

OBSERVAÇÃO III.2.8 A proposição anterior também é válida se $K \leq c \quad(K \geq c)$ e $c \notin K$, já que $K<c \cdot 1=d \quad(K>c \cdot 1=d)$ onde $d \in \omega(z)$.

Demonstração da proposição III.2.7. Mostraremos inicialmente que $K \leq \omega(z)$ e para isto é suficiente mostrar que $z(t) \in V$ para $t$ suficientemente grande, onde $\bar{V}=\{y \in W$ : $y>K\}$.

Como $c=\lim _{k \rightarrow+\infty} z\left(t_{k}\right)$ para alguma seqüência $t_{k} \rightarrow+\infty$; então sendo $V$ aberto (já que $K$ é compacto) e $c \in V$, existe $k_{0}>0$ tal que $z\left(t_{k}\right) \in V$ para todo $k \geq k_{0}$, em particular $K<z\left(t_{k_{0}}\right)$. Como $\Phi_{t}(K)=K$ e o fluxo é fortemente monótono, segue que $K<z\left(t_{k_{0}}+t\right)$ para todo $t \geq 0$, portanto $K \leq \omega(z)$.

Mostraremos agora que $K<\omega(z)$. É certo que $K \cap \omega(z)=\emptyset$, já que se existisse $d \in K \cap \omega(z)$, então $d<c \operatorname{com} d, c \in \omega(z)$ o que contraria a proposição II.1.5.Sejam $a \in K$ e $b \in \omega(z)$; sendo $K$ e $\omega(z)$ invariantes existem $a_{0} \in K, b_{0} \in \omega(z)$ tais que $a=\Phi_{1}\left(a_{0}\right)$, $b=\Phi_{1}\left(b_{0}\right)$; como $K \cap \omega(z)=\emptyset$ e $K \leq \omega(z)$ segue-se que $a_{0} \leq b_{0}$ e $a_{0} \neq b_{0}$, portanto $\Phi_{1}\left(a_{0}\right)<\Phi_{1}\left(b_{0}\right)$ ou seja $a<b ; \operatorname{assim} K<\omega(z)$.

LEMA III.2.9 Sejam $x, y \in W$ tais que $x \leq y, x \neq y$ e $\overline{O_{+}(x)}, \overline{O_{+}(y)}$ são compactos contidos em $W$. Se existe uma seqüencia $t_{k} \rightarrow+\infty$ tal que $\lim _{k \rightarrow+\infty} x\left(t_{k}\right)=a, \lim _{k \rightarrow+\infty} y\left(t_{k}\right)=b$ com $a<b$, então $\omega(x)<\omega(y)$.

OBSERVAÇÃO III.2.10 O lema é válido, se $a \leq b, a \neq b$, já que $\lim _{t_{k} \rightarrow+\infty} y\left(t_{k}+1\right)=$ 
$\Phi_{1}(a)=a \cdot 1 \quad$ e $\lim _{t_{k} \rightarrow+\infty} y\left(t_{k}+1\right)=b \cdot 1 \quad$ e $\quad a \cdot 1<b \cdot 1$.

Demonstração do lema III.2.9 Basta mostrar que $\overline{O_{+}(a)} \leq b$. Donde $\omega(a) \leq b$ e $b \notin \omega(a) \subset \omega(x)$, já que se $b \in \omega(a)$ teríamos $a$ e $b$ em $\omega(x) \operatorname{com} a<b$ que contraria a proposição II.1.5. Aplicando a observação III.2.8 a $K=\omega(a)$ e $b \in \omega(y)$ concluímos que $\omega(a)<\omega(y)$; aplicando novamente a $K=\omega(y) \quad$ e $\quad c \in \omega(a) \subset \omega(x)$, obtemos $\omega(x)<\omega(y)$.

\section{Mostrando que $\overline{O_{+}(a)} \leq b$ :}

Dados $u, v \in W \operatorname{com} u \leq v$ definimos $\xi(u, v)=\sup \left\{r \in \Re^{+}: u \cdot t \leq v\right.$ para todo $\left.t \in[0, r)\right\}$ $\operatorname{assim} 0 \leq \xi(u, v) \leq \infty$.

Como $a<b$, existe algum $\beta>0$ tal que $a \cdot t<b$ para todo $t \in[0, \beta]$, portanto $\xi(a, b) \geq \beta>0$. $\overline{O_{+}(a)} \leq b$.

Mostraremos que $\xi(a, b)=\infty$ o que implica que $a \cdot t \leq b$ para todo $t \geq 0$ donde

Suponhamos que $a$ não seja ponto de equilíbrio e que $\xi(a, b)=T<\infty$.

Temos que $a \cdot s \cdot 1<b \cdot 1$ para $s \in[0, T]$. De fato, para tais $s, a \cdot s \leq b \operatorname{com} a \cdot s \neq b$ ( pois senão teríamos $a, b \in \omega(x)$ com $a<b$ o que contraria a proposição II.1.5) e $\Phi_{1}$ é fortemente monótona.

Seja $\epsilon>0$ tal que $a \cdot s \cdot 1<b \cdot 1$ para $s \in[0, T+\epsilon]$.

Para $k>0$ suficientemente grande tem-se $\xi\left(x \cdot t_{k} \cdot 1, y \cdot t_{k} \cdot 1\right) \geq T+\epsilon$. De fato, existem vizinhanças $U$ de $a \cdot 1 \cdot[0, T+\epsilon]=\{a \cdot 1 \cdot s / s \in[0, T+\epsilon]\}$ e $V$ de $b \cdot 1$ tais que $U<V$ e existe $n_{0} \in N$ tal que $y \cdot t_{k} \cdot 1 \in V$ e $x \cdot t_{k} \cdot 1 \cdot[0, T+\epsilon] \subset U$ para todo $k \geq n_{0}$.

Logo $\lim _{t_{k} \rightarrow+\infty} \sup \xi\left(x \cdot t_{k} \cdot 1, y \cdot t_{k} \cdot 1\right) \geq T+\epsilon$.

Mostraremos que $\xi(x \cdot t, y \cdot t)$ é crescente em $t>0$.

Em primeiro lugar afirmamos que se $u \leq v$, então $\xi(u \cdot t, v \cdot t) \geq \xi(u, v)$ para $t \geq 0$; pois em caso contrário para $\xi(u \cdot t, v \cdot t)<s^{\prime}<\xi(u, v)$ teríamos $u \cdot s \leq v$ para $s \in\left[0, s^{\prime}\right]$, logo $u \cdot(s+t) \leq v \cdot t$ para $t \geq 0$ e, portanto, $\xi(u \cdot t, v \cdot t) \geq s^{\prime}$ o que contraria $\xi(u \cdot t, v \cdot t)<s^{\prime}$. Assim, para $0<t_{1}<t_{2}$, aplicando a afirmação anterior, tem-se $\xi\left(x \cdot t_{2}, y \cdot t_{2}\right) \geq \xi\left(x \cdot t_{1}, y \cdot t_{1}\right)$.

Logo

$$
\lim _{t_{k} \rightarrow+\infty} \xi\left(x \cdot t_{k}, y \cdot t_{k}\right)=\lim _{t \rightarrow+\infty} \xi(x \cdot t, y \cdot t)=
$$




$$
=\lim _{t_{k} \rightarrow+\infty} \sup \xi\left(x \cdot t_{k} \cdot 1, y \cdot t_{k} \cdot 1\right) \geq T+\epsilon .
$$

Por outro lado mostraremos que $\lim _{t_{k} \rightarrow+\infty} \xi\left(x \cdot t_{k}, y \cdot t_{k}\right) \leq T$. Assim teria-se $T \geq T+\epsilon$ com $\epsilon>0$, o que levará a concluir que $T=\xi(a, b)=+\infty$.

Sabemos que $\left(x \cdot t_{k}\right) \cdot s \leq y \cdot t_{k}$ para todo $s \in\left[0, \xi\left(x \cdot t_{k}, y \cdot t_{k}\right)\right]$.

Temos que $\lim _{t_{k} \rightarrow+\infty} \xi\left(x \cdot t_{k}, y \cdot t_{k}\right)<\infty$, pois caso contrário, dado qualquer $n \in N$, existe $k_{0} \in N$ tal que para $k \geq k_{0} \quad \xi\left(x \cdot t_{k}, y \cdot t_{k}\right) \geq n$; assim $\left(x \cdot t_{k}\right) \cdot s \leq y \cdot t_{k}$ para $s \in[0, n]$, logo $T \geq n$ para todo $n \in N$, ou seja, $T=+\infty$, o que contraria a hipótese assumida para $T<\infty$.

Seja $\xi=\lim _{t_{k} \rightarrow+\infty} \xi\left(x \cdot t_{k}, y \cdot t_{k}\right)$ e $0 \leq s<\xi$, para $k$ suficientemente grande tem-se $\xi\left(x \cdot t_{k}, y \cdot t_{k}\right)>s$ e $\left(x \cdot t_{k}\right) \cdot s^{\prime} \leq y \cdot t_{k}$ para $0 \leq s^{\prime} \leq s$, logo $a \cdot s^{\prime} \leq b$, donde segue que $s \leq T$ e portanto $\xi \leqslant T$.

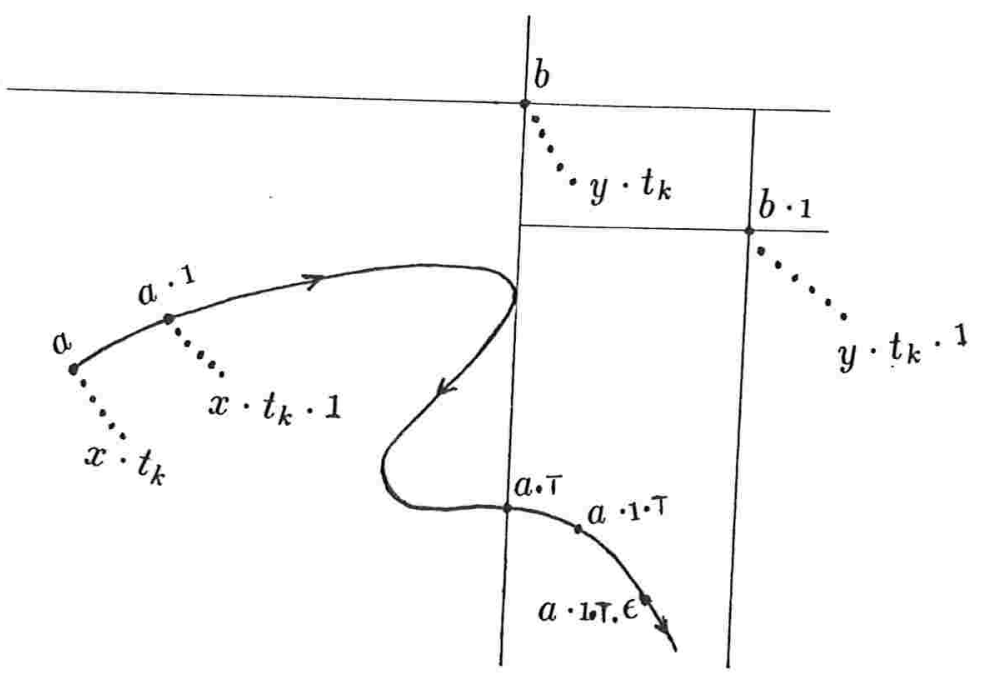

\section{Demonstração do teorema III.2.1}

i) Se $\omega(x)=\omega(y)$, pelo corolário III.2.4, tem-se $\omega(x)=\omega(y) \subset E$.

ii) Se $\omega(x) \neq \omega(y)$ :

1.0 caso $x(t)$ ou $y(t)$ é convergente quando $t \rightarrow+\infty$.

Sem perda de generalidade, suporemos $\lim _{t \rightarrow+\infty} x(t)=p$, isto é, $\omega(x)=\{p\}$. 
Afirmamos que $p \leq \omega(y)$. De fato : dado $q \in \omega(y), q=\lim _{t_{k} \rightarrow+\infty} y\left(t_{k}\right)$ e $x\left(t_{k}\right)<$ $y\left(t_{k}\right)$ pois o fluxo é fortemente monótono e $x \leq y, x \neq y$. Passando ao limite temos $p \leq q$.

Por outro lado $p \notin \omega(y)$, já que em caso contrário, tomando $q \in \omega(y), q \neq p$, teriamos $p \cdot 1<q \cdot 1$ o que contraria a proposição II.1.5.

Logo pela observação III.2.8 do princípio de absorção, tomando $K=\omega(y)$ e $c=p$ tem-se $\omega(x)<\omega(y)$.

$\underline{2.0 \text { caso }} x(t)$ e $y(t)$ não são convergentes quando $t \rightarrow+\infty$.

Sem perda de generalidade, suponhamos que exista $p \in \omega(x)$ tal que $p \notin \omega(y)$, $p=\lim _{t_{k} \rightarrow+\infty} x\left(t_{k}\right)$. Como $x \leq y, x \neq y$ e o fluxo é fortemente monótono tem-se $x\left(t_{k}\right)<y\left(t_{k}\right)$; por outro lado $\left\{y\left(t_{k}\right)\right\} \subset \overline{O_{+}(y)}$ e $\overline{O_{+}(y)}$ é compacto, então existe uma subseqüência $y_{t_{k_{l}}} \rightarrow q$; assim de $x\left(t_{k_{l}}\right)<y\left(t_{k_{l}}\right)$ tem-se $p \leq q$ e, como $p \neq q$, pela observação III.2.10 onclue-se que $\omega(x)<\omega(y)$.

\section{§3. TRAJETÓRIAS QUASE-CONVERGENTES}

Continuamos supondo $W \subset \Re^{n}, W$ aberto, p-convexo, $F$ de classe $C^{1}$ em $W$, cooperativo e irredutível. Também continuamos denotando com $E$ o conjunto dos pontos de equilíbrio de $F$.

Sejam

$W_{c}=\left\{x \in W: \overline{O_{+}(x)} \subset W, \overline{O_{+}(x)}\right.$ compacto $\}, v \in \Re^{n}, v>0$ e $L$ uma reta em $\Re^{n}$ paralela a $v$.

PROPOSIÇÃO III.3.0 Para quase todo $x \in W_{c} \cap L$ tem-se que $\omega(x) \subset E$.

Demonstração. Seja $D=\left\{x \in W_{c}: \omega(x) \not \subset E\right\}$, mostraremos que $D \cap L$ é enumerável.

Para cada $x \in L \cap D$ escolhemos um ponto $z_{x} \in \omega(x)$ e consideramos o conjunto $R=\left\{z_{x}: x \in L \cap D\right\}$.

Dados $x, y \in L \cap D$ podemos supor $x<y$ e, portanto, pelo teorema III.2.1 temos que $z_{x}<z_{y}$. Logo se $L \cap D$ não fosse emumerável, então $R$ não seria e, portanto, conteria um de seus pontos de acumulação, digamos $z_{0}=z_{x_{0}}$ para algum $x_{0} \in L \cap D$; sem perda de generalidade podemos supor que $z_{0}$ é ponto de acumulação de $\left\{z_{x}: x_{0}<x, x \in L \cap D\right\}$. 
Como $\omega\left(x_{0}\right)<\omega(x)$ para $x \in L \cap D$, tal que $x_{0}<x$, tem-se que $\omega\left(x_{0}\right) \leq z_{0}$; logo da irredutibilidade de $F$ e da proposição II.1.5 segue que $\omega\left(x_{0}\right)=\left\{z_{0}\right\} \subset E$ o que contradiz o fato $\omega\left(x_{0}\right) \not \subset E$. Portanto $L \cap D$ é enumerável, logo de medida nula. 口 Como $W_{c}$ e $D$ são borelianos segue da Proposição anterior e do Teorema de Fubini: $\therefore$

TEOREMA $C$ Para quase todo $x \in W_{c}$ tem-se que $\omega(x) \subset E$.

PROPOSIÇÃO III.3.1 Se todos os pontos de equilíbrio são hiperbólicos e $\Phi_{t}$ está definido para todo $t \geq 0$ em $W$, então para quase todo $x \in W_{c}, x(t)$ converge para um poço, quando $t \rightarrow+\infty$.

Demonstração. Já foi mostrado que $D \cap L=\left\{x \in W_{c}: \omega(x) \not \subset E\right\}$ tem medida nula.

Seja:

$$
M=\left\{x \in W_{c}: \omega(x) \subset E \text { e } \omega(x) \text { não é poço }\right\}
$$

Mostraremos que $L \cap M$ é enumerável. Se $x \in L \cap M$, então $\omega(x)$ é um ponto de equilíbrio, pois os pontos de $E$ são isolados. Sejam $x, y$ pontos distintos de $L \cap M$ tais que $x<y$, mostraremos que $\omega(x) \neq \omega(y)$. Suponhamos que $\omega(x)=\omega(y)=\{p\}$. Como para $x<z<y$ com $z \in W$ e $t>0$, tem-se $x(t)<z(t)<y(t)$ e $\lim _{t \rightarrow+\infty} x(t)=p=\lim _{t \rightarrow+\infty} y(t)$, então $z(t) \rightarrow p$ quando $t \rightarrow+\infty$, mas isto não é possível, já que $p$ não é poço. Assim, para pontos $x \neq y$ em $L \cap M$, tem-se que $\omega(x) \neq \omega(y)$ e sendo $E$ enumerável (já que os pontos de equilíbrio são isolados), segue-se que $L \cap M$ é enumerável. Como $M$ é boreliano aplicando Fubini segue que $M$ tem medida nula. 口

DEFINIÇÃO III.3.2 Seja $K \subset W, K \neq \emptyset$. Dizemos que $K$ é um atrator se $K$ é positivamente invariante e existe uma vizinhança $U \subset W$ de $K$ tal que $\overline{O_{+}(x)}$ é compacto, $\overline{O_{+}(x)} \subset W$ e $\omega(x) \subset K$ para todo $x \in U$.

PROPOSIÇÃO III.3.3 Se $K \subset W$ é um atrator compacto, $\Phi_{t}$ definido para todo $t \geq 0$ em $W$ e todos os pontos de equilíbrio em $K$ são hiperbólicos, então $K$ contém um poço.

Demonstração. Mostraremos inicialmente que $K$ contém um ponto de equilíbrio. Suponhamos o contrário, que $K \cap E=\emptyset$. Sendo $K$ atrator existe uma vizinhança $U$ de $K$ tal que $\emptyset \neq \omega(x) \subset K$ para todo $x \in U$. Como $\omega(x) \cap E=\emptyset$ e $\omega(x)$ compacto, temos $U \subset D=\left\{x \in W_{c}: \omega(x) \not \subset E\right\}$, isto implica que $U$ tem medida nula, o que é falso, já que $U$ é uma vizinhança, portanto $K \cap E \neq \emptyset$. 
Mostraremos agora que $K$ contém um poço: pelo lema de Zorn, $K \cap E$, parcialmente ordenado pela relação $\leq$, tem um elemento maximal $p$; isto é, se existir $q \in K \cap E$ tal que $q \geq p$, então $q=p$. Sejam $B$ uma bola aberta tal que $p \in B \subset U$ e $V=B \cap\{y: y>$ $p\} \subset U$, então pelo teorema $C$, existe $y \in V$ tal que $\omega(y) \subset E$. Como $y>p$, portanto $y(t)>p$ para todo $t>0$, tem-se $p \leq \omega(y) \subset K \cap E$, logo $q=p$ para todo $q \in \omega(y)$ de onde $\omega(y)=\{p\}$, assim $\lim _{t \rightarrow+\infty} y(t)=p$. Deste modo escolhendo pontos $y_{1}, y_{2} \in V$ tais que $y_{2}>y_{1}>p$ e $\lim _{t \rightarrow+\infty} y_{i}(t)=p$, temos que $x(t) \rightarrow p$ para $x \in W$ com $y_{1}<x<y_{2}$ quando $t \rightarrow+\infty$ e como $p$ é hiperbólico, então $p$ é um poço.

APLICAÇÃO Se $F: \Re^{n} \rightarrow \Re^{n}$ é um campo vetorial de classe $C^{1}$, cooperativo em $\Re_{+}^{n}$ e irredutível em int $\Re_{+}^{n}$, tal que:

(i) $F(0) \geq 0$

(ii) $\Phi_{t}$ definido para todo $t \geq 0$ em $\Re_{+}^{n}$

(iii) para cada $x \in \Re_{+}^{n}(x \geq 0)$, existe $y>x \operatorname{com} F(y)<0$. Além disso se $x>0$, existe $x^{\prime}>0$ tal que $x^{\prime}<x$ e $F\left(x^{\prime}\right)>0$.

Então, $\Re_{+}^{n}$ é positivamente invariante e para $x \in \Re_{+}^{n} \quad \overline{O_{+}(x)}$ é compacto. Além disso para quase todo ponto $x \in \Re_{+}^{n}, \omega(x) \subset E$.

De fato:

Seja $x \geq 0$, por (iii) existe $y>x \operatorname{com} F(y)<0$. Como $F$ é cooperativo em $\Re_{+}^{n}$, então $\Phi_{t}(0) \leq \Phi_{t}(x) \leq \Phi_{t}(y), D \Phi_{t}(0) \geq 0$ e $D \Phi_{t}(y) \geq 0$ para todo $t>0$. Sendo $D \Phi_{t}(0) F(0)=F\left(\Phi_{t}(0)\right), D \Phi_{t}(y) F(y)=F\left(\Phi_{t}(y)\right)$ para todo $t>0, F(0) \geq 0$ e $F(y)<0$, segue-se que $F\left(\Phi_{t}(0)\right) \geq 0$ e $F\left(\Phi_{t}(y)\right)<0$ para todo $t>0$; portanto $\Phi_{t}(0)$ é crescente e $\Phi_{t}(y)$ é decrescente. Logo $0=\Phi_{0}(0) \leq \Phi_{t}(0) \leq \Phi_{t}(x) \leq \Phi_{t}(y) \leq \Phi_{0}(y)=y$ o que implica que $0 \leq \Phi_{t}(x) \leq y$ para todo $t \geq 0$.

Assim, provamos que $\Re_{+}^{n}$ é positivamente invariante e $\overline{O_{+}(x)}$ é compacto contido em $\Re_{+}^{n}$ para todo $x \in \Re_{+}^{n}$. Além disso para $x>0$, tomando $x^{\prime}$ tal que $0<x^{\prime}<x$ com $F\left(x^{\prime}\right)>0$ e repetindo o mesmo argumento anterior concluímos que $\overline{O_{+}(x)} \subset$ int $\Re_{+}^{n}$, tendo em conta que o sistema é irredutível no int $\Re_{+}^{n}$. Donde concluímos, aplicando o teorema $\mathrm{C} \operatorname{com} W=\operatorname{int} \Re_{+}^{n}$, que para quase todo ponto $x \in \Re_{+}^{n}, \omega(x) \subset E$. forma:

Um exemplo deste tipo de sistemas é um "positive feedback loop" de $n$ espécies da

$$
\left\{\begin{array}{l}
\dot{x_{1}}=f\left(x_{n}\right)-A_{1} x_{1} \\
\dot{x_{j}}=x_{j-1}-A_{j} x_{j}, \quad j=2, \ldots, n
\end{array}\right.
$$


com $A_{j}>0$ para $j=1,2, \ldots, n$ e onde $f: \Re \rightarrow \Re$ é uma função de classe $C^{1}$ tal que $f(s), f^{\prime}(s)>0$ para todo $s>0$ e $0 \leq f^{\prime}(s)<M$ para todo $s \geq 0$ onde $M$ é uma constante positiva.

(Ò̉bservar que a condição $0 \leq f^{\prime}(s)<M$ foi imposta para assegurar que o campo seja globalmente lipchitziano e, portanto, o fluxo esteja definido para todo $t$.)

Veremos que este sistema é cooperativo em $\Re_{+}^{n}$ e irredutível em int $\Re_{+}^{n}$ e satisfaz as condições (i), (ii), (iii) anteriores; mais ainda, mostraremos que para quase todo ponto $x \in \Re_{+}^{n}, x(t)$ converge a um ponto de equilíbrio quando $t \rightarrow+\infty$.

Neste exemplo, o campo $F=\left(F_{1}, \ldots, F_{n}\right): \Re^{n} \rightarrow \Re^{n}$ é dado por:

$$
\begin{aligned}
& F_{1}(x)=f\left(x_{n}\right)-A_{1} x_{1} \\
& F_{j}(x)=x_{j-1}-A_{j} x_{j} \text { para } j=2, \ldots, n
\end{aligned}
$$

portanto

$$
D F(x)=\left[\begin{array}{ccccccc}
-A_{1} & 0 & 0 & \cdot & 0 & 0 & f^{\prime}\left(x_{n}\right) \\
1 & -A_{2} & 0 & . & \cdot 0 & 0 & \\
0 & 1 & -A_{3} & . & . & 0 & 0 \\
\vdots & \vdots & \vdots & \vdots & \vdots & \vdots & \vdots \\
0 & 0 & 0 & . & \cdot & 1 & -A_{n}
\end{array}\right]
$$

que é uma matriz irredutível para todo $x \in \operatorname{int} \Re_{+}^{n}$ e como $\frac{\partial F_{i}}{\partial x_{j}}(x) \geq 0$ para todo $i \neq j$ e $x \in \Re_{+}^{n}$, então o sistema é cooperativo em $\Re_{+}^{n}$.

(i) $F(0) \geq 0$, já que $F(0)=(f(0), 0, \ldots, 0)$ e $f(0) \geq 0$ (pois $f$ é contínua e $f(s) \geq 0$ para $s>0$ )

(ii) $F$ é lipchitziana em $\Re_{+}^{n}$

(iii) Para cada $x=\left(x_{1}, \ldots, x_{n}\right) \in \Re_{+}^{n}$, existe $y>x$ tal que $F(y)<0$, pois dado $x \in \Re_{+}^{n}$, pode-se escolher $y=\left(y_{1}, \ldots, y_{n}\right)$ do seguinte modo: tomando $y_{n}>x_{n}$ e escolhendo $y_{1}>x_{1}$ tal que $A_{1} y_{1}>f\left(y_{n}\right)$, em seguida tomando $y_{2}>x_{2}$ tal que $A_{2} y_{2} \geq y_{1}$ e, assim 
sucessivamente. Analogamente para $x>0$ pode-se escolher $0<x^{\prime}<x \operatorname{com} F\left(x^{\prime}\right)>0$.

Afirmamos que $x(t)$ converge para um ponto de equilíbrio para quase todo ponto de $\Re_{+}^{n}$. De fato:

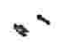

como

$$
E \subset\left\{r\left(A_{2}, 1, \frac{1}{A_{3}}, \frac{1}{A_{3} A_{4}}, \ldots, \frac{1}{A_{3} A_{4} \ldots A_{n}}\right) / r \in \Re\right\}
$$

então $E$ é totalmente ordenado. Dado $x \in \Re_{+}^{n}$, tal que $\omega(x) \subset E$ mostraremos que $\omega(x)$ é um ponto de equilíbrio.

Suponhamos que $\omega(x)$ contivesse dois pontos diferentes $p$ e $q$. Seja $p \leq q$ (já que $E$ é totalmente ordenado), então $p \cdot 1<q \cdot 1 \mathrm{com} p \cdot 1, q \cdot 1 \mathrm{em} \omega(x)$, o que contraria a proposição II.1.5. 口 


\section{CAPÍTULO IV. UM EXEMPLO DE COMPETIÇÃO}

\section{ENTRE TRÊS ESPÉCIES}

Consideremos o seguinte sistema de equações:

$$
\left\{\begin{array}{l}
\dot{x_{1}}=x_{1}\left(1-x_{1}-\alpha x_{2}-\beta x_{3}\right) \\
\dot{x_{2}}=x_{2}\left(1-\beta x_{1}-x_{2}-\alpha x_{3}\right) \\
\dot{x_{3}}=x_{3}\left(1-\alpha x_{1}-\beta x_{2}-x_{3}\right)
\end{array}\right.
$$

em $\Re_{+}^{3}=\left\{\left(x_{1}, x_{2}, x_{3}\right) \in \Re^{3}: x_{i} \geq 0, i=1,2,3\right\}$, com parâmetros $\alpha \geq 0, \beta \geq 0$. Esta é uma equação de Gauss - Lotka - Volterra modelando competição de três espécies de população cujas densidades são $x_{1}, x_{2}$ e $x_{3}$ respectivamente.

De acordo com a variação dos parâmetros $\alpha$ e $\beta$ o sistema (1) tem dinâmicas diferentes. Exporemos com detalhes os casos especiais $\alpha+\beta=2 \operatorname{com} \alpha \neq \beta, \alpha>0, \beta>0$ e $\alpha+\beta>2 \operatorname{com} 0<\alpha<1<\beta$, estudados por May e Leonard [13]. No primeiro caso, o interior do simplexo $x_{1}+x_{2}+x_{3}=1, x_{i} \geq 0, i=1,2,3$ é união de órbitas periódicas concêntricas em torno do ponto de equilíbrio $\frac{1}{3}(1,1,1)$ e todo ponto do interior de $\Re_{+}^{3}$ tem como $\omega$-limite uma dessas órbitas, exceto os da reta $x_{1}=x_{2}=x_{3}$, invariante, que convergem ao ponto $\frac{1}{3}(1,1,1)$ (Ver figura A) No segundo caso, todos os pontos do interior de $\Re_{+}^{3}$, exceto os da reta $x_{1}=x_{2}=x_{3}$, invariante, (que convergem ao ponto $\frac{1}{1+\alpha+\beta}(1,1,1$ )

tem como $\omega$-limite um ciclo contido na fronteira de $\Re_{+}^{3}$, unindo os pontos de equilíbrio $(1,0,0),(0,1,0)$ e $(0,0,1)$. May e Leonard descreveram este ciclo de maneira imprecisa, sendo melhorada por Schuster e Wolff [22].

Nesses casos mencionados observar que os conjuntos $\omega$ - limites estão numa posição especial tal que não admite dois pontos relacionados por $<$.

Segundo a variação de $\alpha$ e $\beta$ os pontos críticos são:

(i) $(0,0,0),(1,0,0),(0,1,0),(0,0,1)$ e $\frac{1}{1+\alpha+\beta}(1,1,1)$ para $1 \leq \alpha, 0 \leq \beta \leq 1$ e $\alpha \neq \beta$ ou $1 \leq \beta, 0 \leq \alpha \leq 1$ e $\alpha \neq \beta$.

(ii) $(0,0,0),(1,0,0),(0,1,0),(0,0,1), \frac{1}{1+\alpha+\beta}(1,1,1),\left(\frac{1-\alpha}{1-\beta \alpha}, \frac{1-\beta}{1-\beta \alpha}, 0\right),\left(0, \frac{1-\alpha}{1-\beta \alpha}, \frac{1-\beta}{1-\beta \alpha}\right)$

e $\left(\frac{1-\beta}{1-\alpha \beta}, 0, \frac{1-\alpha}{1-\beta \alpha}\right)$ para $0 \leq \alpha<1$ e $0 \leq \beta<1$, ou $\alpha>1$ e $\beta>1$.

(iii) $(0,0,0)$ e todos os pontos do simplexo $x_{1}+x_{2}+x_{3}=1, x_{i} \geq 0, i=1,2,3$ se 
$\alpha=\beta=1$.
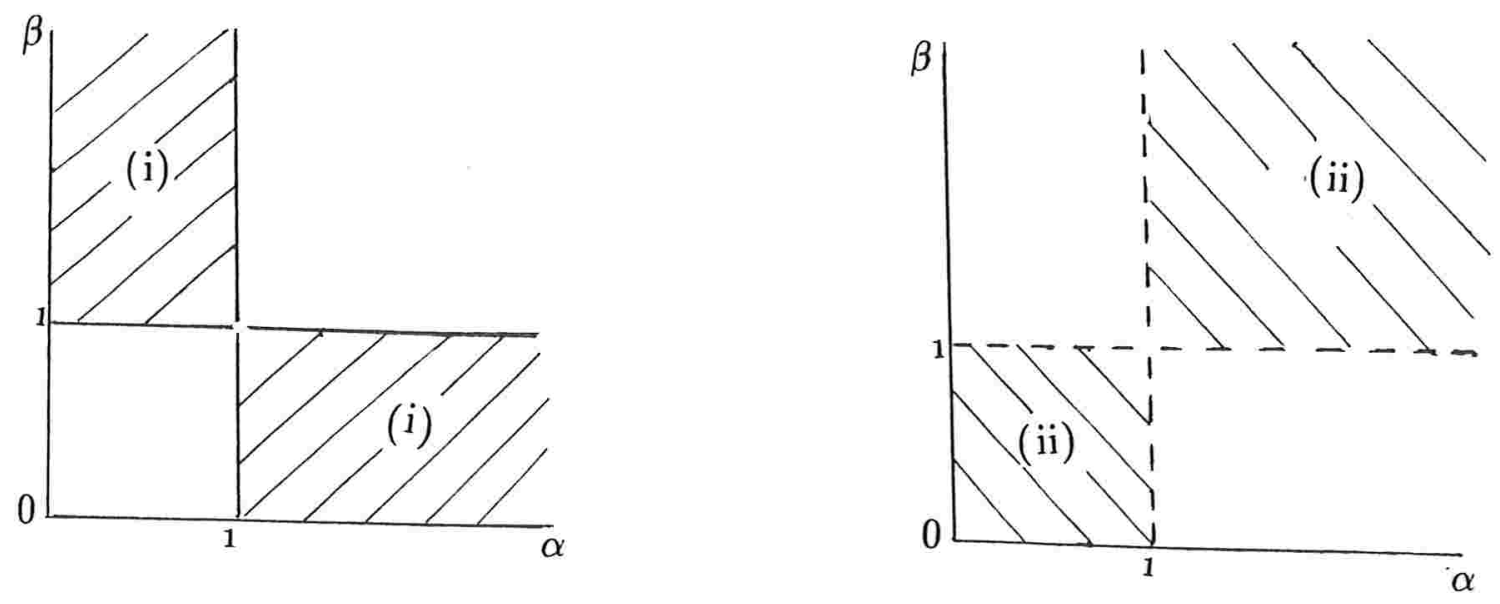

Vemos que:

$D F(0,0,0)=\left(\begin{array}{lll}1 & 0 & 0 \\ 0 & 1 & 0 \\ 0 & 0 & 1\end{array}\right) \log \lambda=1$ é único autovalor, portanto $(0,0,0)$ é sempre uma fonte.

As matrizes

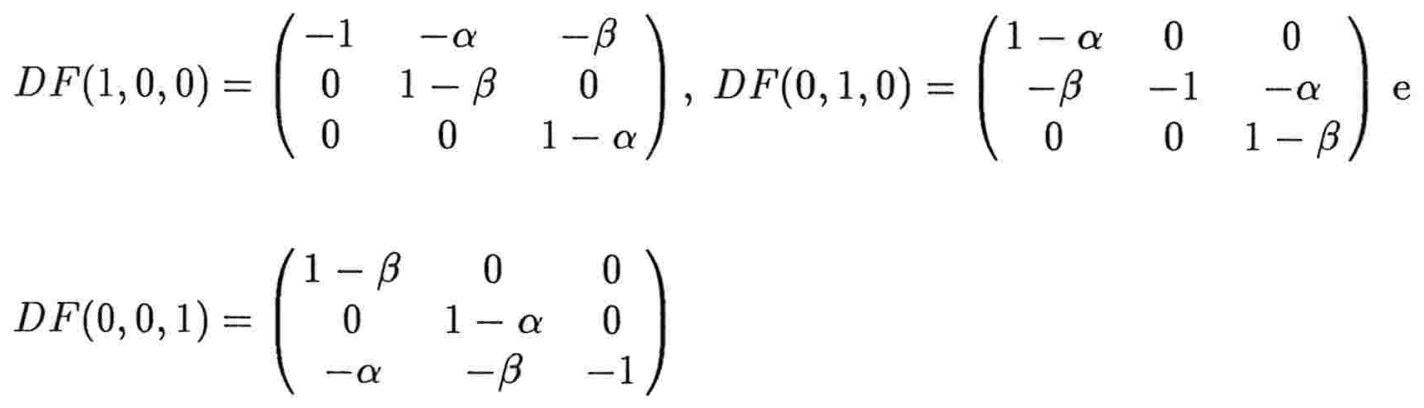

tem por autovalores $-1,1-\alpha$ e $1-\beta$. 
Mostramos abaixo uma tabela dos autovalores e autoespaços para cada matriz, segundo a variação de $\alpha$ e $\beta$.

AUTO VARIAÇÃO DOS

BASE DO AUTO ESPAÇO DA MATRIZ

VALOR PARÂMETROS

\begin{tabular}{|c|c|c|c|c|c|c|}
\hline $\begin{array}{l}\text { VALO } \\
\mid-1\end{array}$ & $\mathrm{P} A$ & $\begin{array}{l}\text { RAME } \\
+?+2\end{array}$ & ROS & $D F(1,0,0)$ & $D F(0,1,0)$ & $D F(0,0,1)$ \\
\hline & & $\alpha=2$ & $\beta \neq 0$ & $(1,0,0)$ & $(0,1,0)$ & $(0,0,1)$ \\
\hline & $\alpha \neq \beta$ & $\alpha=2$ & $\beta=0$ & $(1,0,0),(0,0,1)$ & $(1,0,0),(0,1,0)$ & $(0,1,0),(0,0,1)$ \\
\hline & & $\alpha \neq 0$ & $\beta=2$ & $(1,0,0)$ & $(0,1,0)$ & $(0,0,1)$ \\
\hline & & $\alpha=0$ & $\beta=2$ & $(1,0,0),(0,1,0)$ & $(0,1,0),(0,0,1)$ & $(1,0,0),(0,0,1)$ \\
\hline & & $\alpha=2$ & $\beta=2$ & $(1,0,0),(0,1,-1)$ & $(1,0,-1),(0,1,0)$ & $(1,-1,0),(0,0,1)$ \\
\hline & $\alpha=\beta$ & $\alpha \neq 2$ & $\beta \neq 2$ & $(1,0,0)$ & $(0,1,0)$ & $(0,0,1)$ \\
\hline & & $\alpha=0$ & $\beta \neq 2$ & $(0, \beta-2,0)$ & $(0,0, \beta-2)$ & $(\beta-2,0,0)$ \\
\hline & $\alpha \neq \beta$ & $\alpha \neq 0$ & $\beta \neq 2$ & $(\alpha, \beta-2,0)$ & $(0, \alpha, \beta-2)$ & $(\beta-2,0, \alpha)$ \\
\hline $1-\beta$ & & $\alpha=0$ & $\beta=2$ & $(1,0,0),(0,1,0)$ & $(0,1,0),(0,0,1)$ & $(1,0,0),(0,0,1)$ \\
\hline & & $\alpha \neq 0$ & $\beta=2$ & $(1,0,0)$ & $(0,1,0)$ & $(0,0,1)$ \\
\hline & $\alpha=\beta$ & $\alpha=2$ & $\beta=2$ & $(1,0,0),(0,1,-1)$ & $(1,0,-1),(0,1,0)$ & $(1,-1,0),(0,0,1)$ \\
\hline & & $\alpha \neq 2$ & $\beta \neq 2$ & $\left(\frac{\beta}{\beta-2}, 1,0\right),\left(\frac{\beta}{\beta-2}, 0,1\right)$ & $\left(1, \frac{\beta}{\beta-2}, 0\right),\left(0, \frac{\beta}{\beta-2}, 1\right)$ & $\left(1,0, \frac{\beta}{\beta-2}\right),\left(0,1, \frac{\beta}{\beta-2}\right)$ \\
\hline & & $\alpha \neq 2$ & $\beta=0$ & $(0,0, \alpha-2)$ & $(\alpha-2,0,0)$ & $(0, \alpha-2,0)$ \\
\hline & $\alpha \neq \beta$ & $\alpha \neq 2$ & $\beta \neq 0$ & $(\beta, 0, \alpha-2)$ & $(\alpha-2, \beta, 0)$ & $(0, \alpha-2, \beta)$ \\
\hline & & $\alpha=2$ & $\beta=0$ & $(1,0,0),(0,0,1)$ & $(1,0,0),(0,1,0)$ & $(0,1,0),(0,0,1)$ \\
\hline $1-\alpha$ & & $\alpha=2$ & $\beta \neq 0$ & $(1,0,0)$ & $(0,1,0)$ & $(0,0,1)$ \\
\hline & $\alpha=\beta$ & $\alpha=2$ & $\beta=2$ & $(1,0,0),(0,1,-1)$ & $(1,0,-1),(0,1,0)$ & $(-1,1,0),(0,0,1)$ \\
\hline & & $\alpha \neq 2$ & $\beta \neq 2$ & $\left(\frac{\alpha}{\alpha-2}, 1,0\right),\left(\frac{\alpha}{\alpha-2}, 0,1\right)$ & $\left(1, \frac{\alpha}{\alpha-2}, 0\right),\left(0, \frac{\alpha}{\alpha-2}, 1\right)$ & $\left(1,0, \frac{\alpha}{\alpha-2}\right),\left(0,1, \frac{\alpha}{\alpha-2}\right)$ \\
\hline
\end{tabular}


A matriz $D F\left(\frac{1}{1+\alpha+\beta}(1,1,1)\right)=-\frac{1}{1+\alpha+\beta}\left(\begin{array}{ccc}1 & \alpha & \beta \\ \beta & 1 & \alpha \\ \alpha & \beta & 1\end{array}\right)$ tem como autovalores $-1 \mathrm{e}$ $\frac{1}{1+\alpha+\beta}\left(\frac{\alpha+\beta-2}{2}\right) \pm \frac{\alpha-\beta}{1+\alpha+\beta}\left(\frac{\sqrt{3}}{2}\right) i$. O autovalor -1 tem por autovetor $(1,1,1)$ e as trajetórias que passam pela semi-reta $\{r(1,1,1): r>0\}$ permanecem nela e tendem para $p=\frac{1}{1+\alpha+\beta}(1,1,1)$ quando $t \rightarrow+\infty$ (pois $F(y, y, y)=(z, z, z)$ onde $\left.z=y[1-y(1+\alpha+\beta)]\right)$ para quaisquer $\alpha \geq 0, \beta \geq 0$.

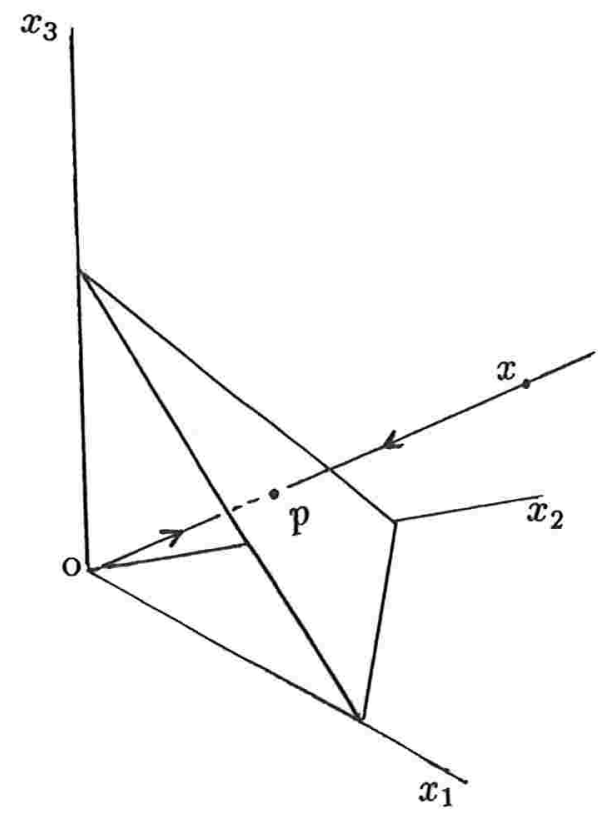

Como $F\left(x_{1}, 0,0\right)=\left(x_{1}\left(1-x_{1}\right), 0,0\right), F\left(0, x_{2}, 0\right)=\left(0, x_{2}\left(1-x_{2}\right), 0\right)$ e $F\left(0,0, x_{3}\right)=$ $\left(0,0, x_{3}\left(1-x_{3}\right)\right)$, então os semi-eixos coordenados positivos são sempre invariantes.

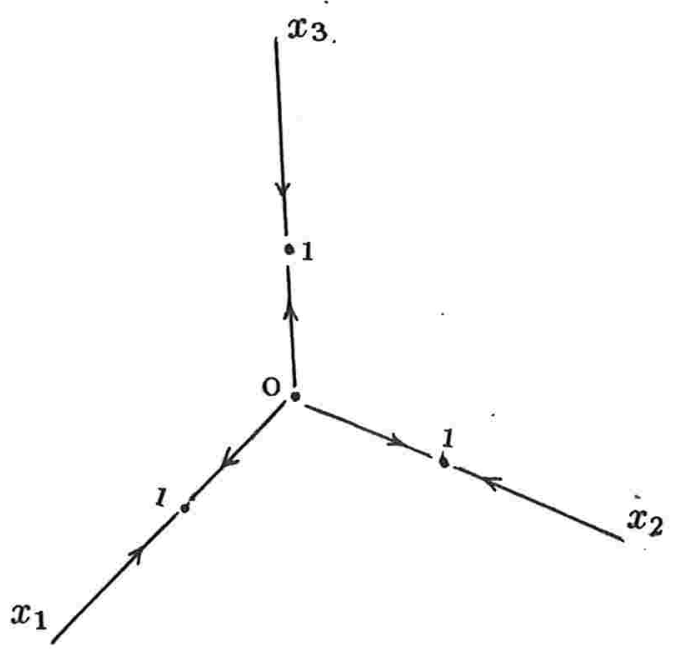


Tem-se também que

$F\left(x_{1}, x_{2}, 0\right)=\left(x_{1}\left(1-x_{1}-\alpha x_{2}\right), x_{2}\left(1-\beta x_{1}-x_{2}\right), 0\right)$

$F\left(0, x_{2}, x_{3}\right)=\left(0, x_{2}\left(1-x_{2}-\alpha x_{3}\right), x_{3}\left(1-\beta x_{2}-x_{3}\right)\right)$

$F\left(x_{1}, 0, x_{3}\right)=\left(x_{1}\left(1-x_{1}-\beta x_{3}\right), 0, x_{3}\left(1-\alpha x_{1}-x_{3}\right)\right)$.

Portanto, os planos coordenados no primeiro octante são sempre invariantes. Daí segue que o interior de $\Re_{+}^{3}$ também é sempre invariante. na figura

Estudaremos detalhadamente os casos especiais de parâmetros da região hachurada

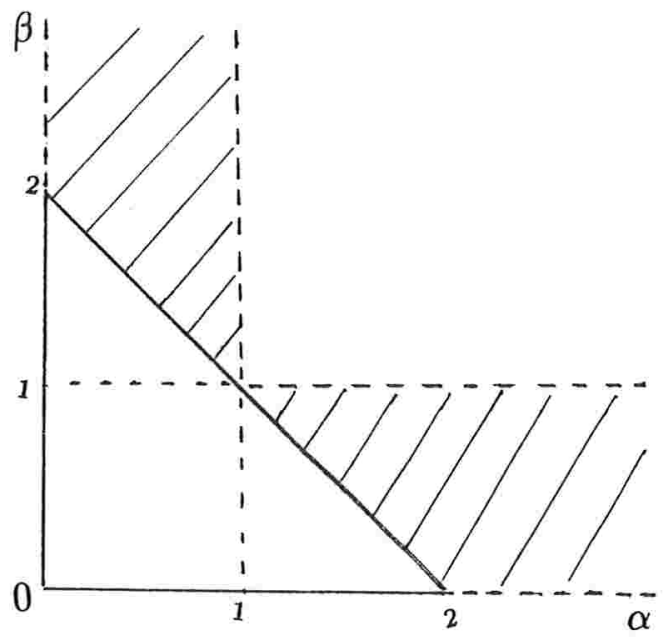

Primeiro caso $: \alpha+\beta=2, \alpha>0, \beta>0$

a) $\alpha=\beta=1$

Seja $x=\left(x_{1}, x_{2}, x_{3}\right) \in \Re_{+}^{3}, x \neq 0$ e $\lambda \in \Re$ tal que $\left(x_{1}, x_{2}, x_{3}\right)=(\lambda a, \lambda b, \lambda c)$ com $a+b+c=1$. Como $F\left(x_{1}, x_{2}, x_{3}\right)=(\lambda(1-\lambda) a, \lambda(1-\lambda) b, \lambda(1-\lambda) c)=\lambda(1-\lambda)(a, b, c)$ então a solução do sistema passando por $x \in \Re_{+}^{3}$ está contida na semi-reta $0 x$ e tem como 
$\omega$-limite o ponto crítico $(a, b, c)$.

b) $\alpha \neq \beta$

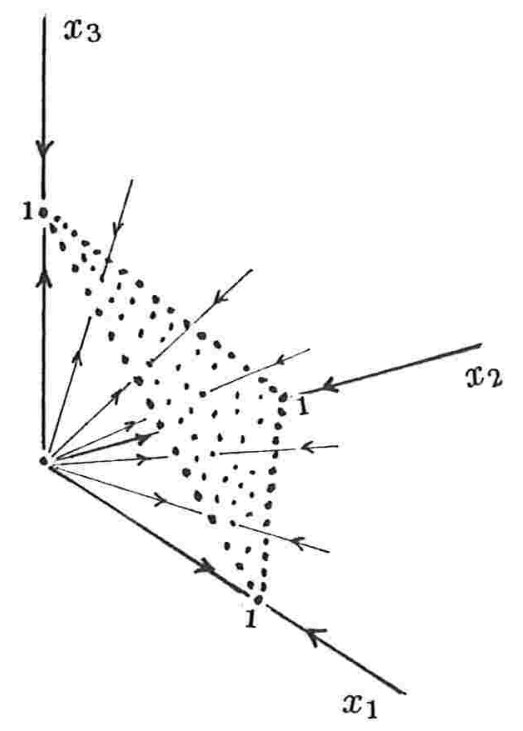

Suporemos sem perda de generalidade que $\alpha>1$. Primeiro faremos um esboço do gráfico das trajetórias na fronteira de $\Re_{+}^{3}$. Para isto levaremos em conta a natureza dos pontos críticos $(0,0,0),(1,0,0),(0,1,0)$ e $(0,0,1)$, a invariância dos planos coordenados no primeiro octante e os resultados a seguir.

Como $\alpha+\beta=2$, tem-se

$F\left(x_{1}, x_{2}, 0\right) \cdot(1,1,0)=\dot{x_{1}}+\dot{x_{2}}=\left(x_{1} x_{2}(1-\alpha), x_{1} x_{2}(1-\beta), 0\right) \cdot(1,1,0)=0$ se $x_{1}+x_{2}=1$,

$F\left(x_{1}, 0, x_{3}\right) \cdot(1,0,1)=\dot{x_{1}}+\dot{x_{3}}=\left(x_{1} x_{3}(1-\beta), 0, x_{1} x_{3}(1-\alpha)\right) \cdot(1,0,1)=0$ se $x_{1}+x_{3}=1$,

$F\left(0, x_{2}, x_{3}\right) \cdot(0,1,1)=\dot{x_{2}}+\dot{x_{3}}=\left(0, x_{2} x_{3}(1-\alpha), x_{2} x_{3}(1-\beta)\right) \cdot(0,1,1)=0$ se $x_{2}+x_{3}=1$,

logo os segmentos de reta unindo os pares de pontos de $\{(1,0,0),(0,1,0),(0,0,1)\}$ são invariantes. 
Mostra-se abaixo o retrato de fase nos planos coordenados.
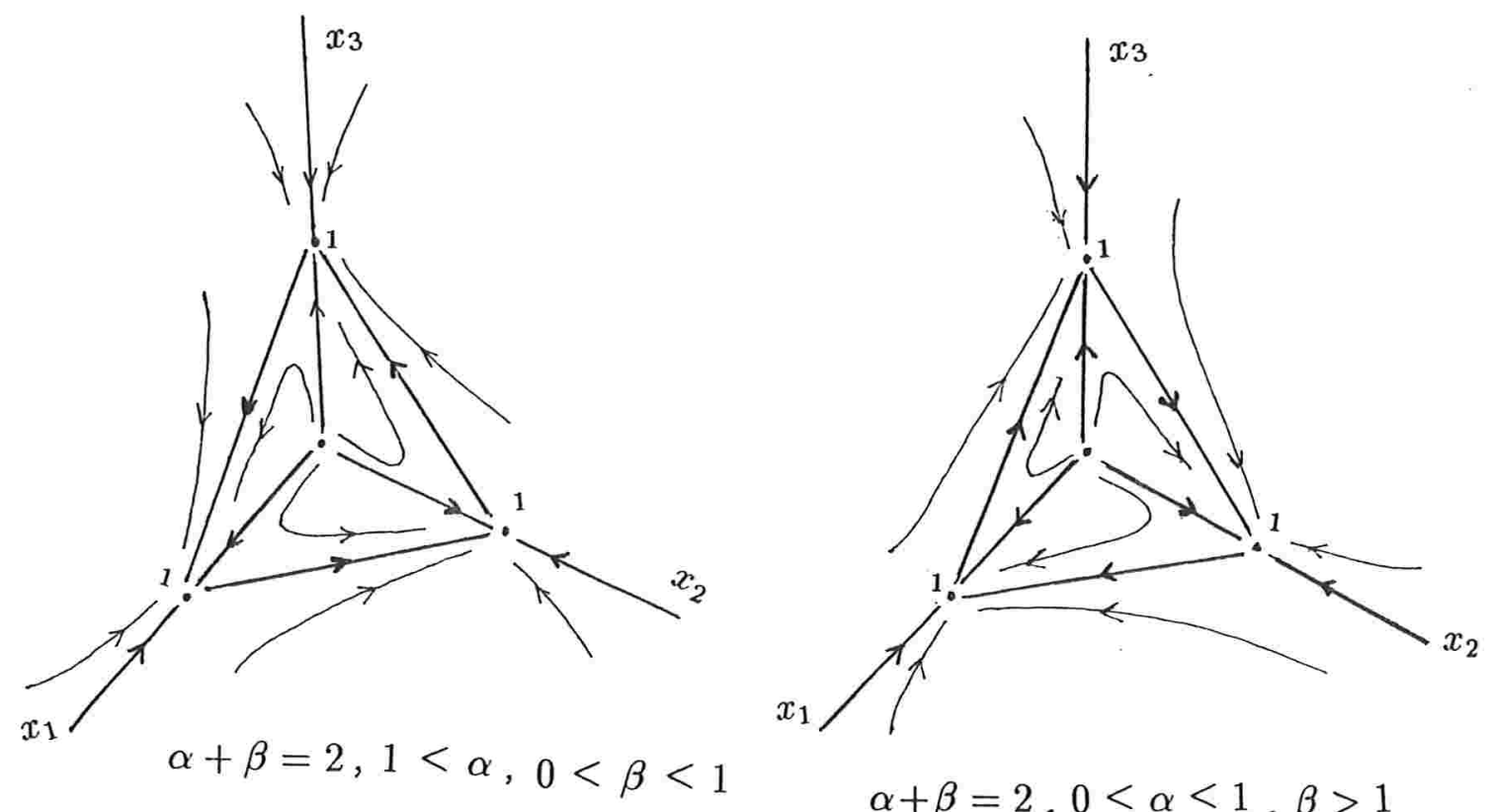

$$
\alpha+\beta=2,0<\alpha<1, \beta>1
$$

Descreveremos agora os conjuntos $\omega$-limite dos pontos do interior de $\Re_{+}^{3}$, com exceção dos pontos $\left(x_{1}, x_{2}, x_{3}\right)$ com $x_{1}=x_{2}=x_{3}$, que conforme já vimos tem como $\omega$-limite o ponto $\frac{1}{3}(1,1,1)$.

Sendo $x(t)=\left(x_{1}(t), x_{2}(t), x_{3}(t)\right)$ a solução que no instante $t=0$ passa por um ponto dado $x=\left(x_{1}^{0}, x_{2}^{0}, x_{3}^{0}\right)$ interior a $\Re_{+}^{3}$.

Definimos

$$
S(t)=x_{1}(t)+x_{2}(t)+x_{3}(t) \text { e } P(t)=x_{1}(t) x_{2}(t) x_{3}(t)
$$

como

$$
\dot{S}=x_{1}+x_{2}+x_{3}-\left[\left(x_{1}^{2}+x_{2}^{2}+x_{3}^{2}\right)+(\alpha+\beta) x_{1} x_{2}+(\alpha+\beta) x_{2} x_{3}+(\alpha+\beta) x_{1} x_{3}\right]
$$


e $\alpha+\beta=2$, então

$$
\dot{S}=S-S^{2}=S(1-S)
$$

de onde segue que o simplexo $H=\left\{\left(x_{1}, x_{2}, x_{3}\right) \in \Re_{+}^{3}: x_{1}+x_{2}+x_{3}=1\right\}$ é invariante, $S(t)$ estritamente crescente se $0<S(0)<1$ e estritamente decrescente se $S(0)>1$.

$$
\text { Além disso como } S(t)=\frac{S(0)}{S(0)+(1-S(0)) e^{-t}},
$$

então $x(t)$ aproxima-se do simplexo $H$ quando $t \rightarrow+\infty$.

Por outro lado, no interior de $\Re_{+}^{3}$ tem-se que

$$
\frac{1}{x_{1}} \dot{x_{1}}+\frac{1}{x_{2}} \dot{x_{2}}+\frac{1}{x_{3}} \dot{x_{3}}=3-(1+\alpha+\beta)\left(x_{1}+x_{2}+x_{3}\right)=3(1-S),
$$

ou seja

$$
\frac{d}{d t}[\ln P(t)]=3(1-S)=3 \frac{\dot{S}}{S}=\frac{d}{d t}\left(\ln S^{3}\right)
$$

o que implica que

$$
P(t)=c_{0} S^{3}(t) \text { onde } c_{0}=\frac{p_{0}}{s_{0}^{3}}
$$

sendo $p_{0}=P(0)$ e $s_{0}=S(0)$. Assim

$$
\lim _{t \rightarrow+\infty} P(t)=\frac{p_{0}}{s_{0}^{3}}
$$

Por (2) e (4) tem-se que quando $t \longrightarrow+\infty$ os pontos da trajetória $x(t)$ aproximamse da curva $\gamma$, intersecção do simplexo $H$ com o hiperbolóide $x_{1} x_{2} x_{3}=\frac{p_{0}}{s_{0}^{3}}=c_{0}$.

Como $\max \left\{x_{1} x_{2} x_{3}: x_{1}+x_{2}+x_{3}=s_{0}, x_{i} \geq 0, i=1,2,3\right\}=\frac{s_{0}^{3}}{27}$ que é atingido no ponto $\frac{s_{0}}{3}(1,1,1)$, então $p_{0} \leq \frac{s_{0}^{3}}{27}$ e $0<c_{0} \leq \frac{1}{27}$.

Mostraremos inicialmente que esta interseção $\gamma$ é uma curva fechada para $0<c_{0}<\frac{1}{27}$ e um ponto para $c_{0}=\frac{1}{27}$. Para isto basta mostrar que sua projeção sobre o plano $x_{1} x_{2}$ :

$$
x_{1} x_{2}\left(1-x_{1}-x_{2}\right)=c_{0}
$$

é uma curva fechada ou um ponto, contida na região $D=\left\{\left(x_{1}, x_{2}\right): x_{1}+x_{2}<1\right.$, $\left.x_{i}>0, i=1,2\right\}$. 
De (5) segue que

$$
x_{2}=\frac{1-x_{1}}{2} \pm \frac{1}{2} \sqrt{\frac{g\left(x_{1}\right)}{x_{1}}}
$$

onde $g\left(x_{1}\right)=x_{1}\left(x_{1}-1\right)^{2}-4 c_{0}$.

As figuras abaixo mostram um esboço da variação dos gráficos de $g$ sobre o intervalo aberto $(0,1)$ para $0<c_{0}<\frac{1}{27}$ e um esboço das curvas (5).
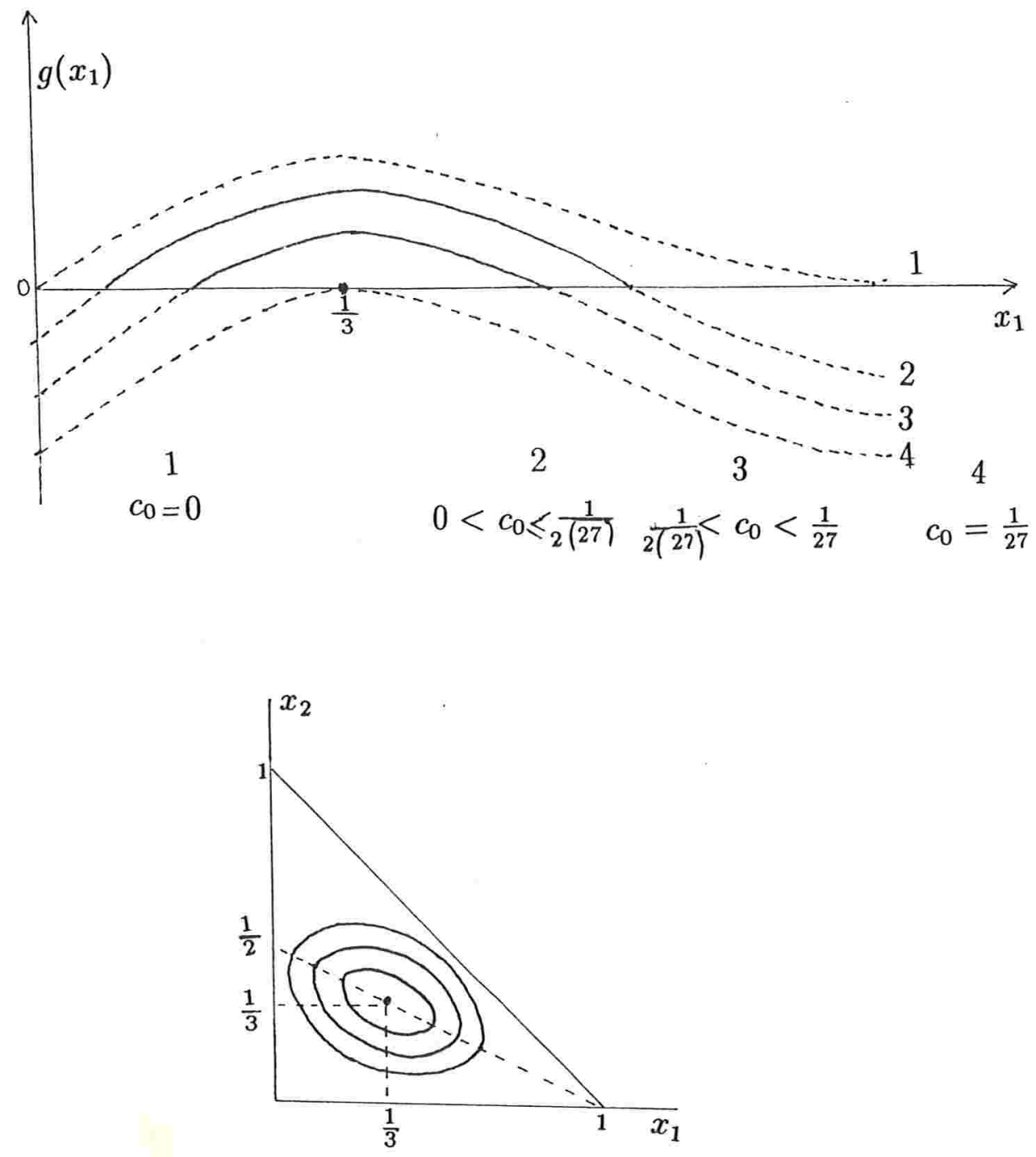

Pelo que já vimos $\emptyset \neq \omega(x) \subset H$.

Mostraremos agora que $\omega(x)=\gamma$ e que $\gamma$ é uma órbita periódica. 
Seja $y \in \omega(x)$ e $x\left(t_{n}\right) \rightarrow y$ quando $t_{n} \rightarrow+\infty$, como $\frac{P\left(t_{n}\right)}{S^{3}\left(t_{n}\right)}=c_{0}$ por (3), tomando limite quando $t_{n} \rightarrow+\infty, \frac{y_{1} y_{2} y_{3}}{y_{1}+y_{2}+y_{3}}=c_{0}$.

Por outro lado

$$
1=\lim _{t_{n} \rightarrow+\infty} S\left(t_{n}\right)=y_{1}+y_{2}+y_{3}
$$

portanto, $y_{1} y_{2} y_{3}=c_{0} ; \operatorname{assim} y \in \gamma$.

Como $O_{+}(y) \subset \omega(x) \subset \gamma$ e $\gamma$ não contém pontos de equilíbrio, então $O_{+}(y)=\gamma$. Donde concluímos que $\omega(x)=\gamma$ e $\gamma$ é uma órbita periódica.

A figura abaixo mostra a maneira como a trajetória tende ao ciclo limite.

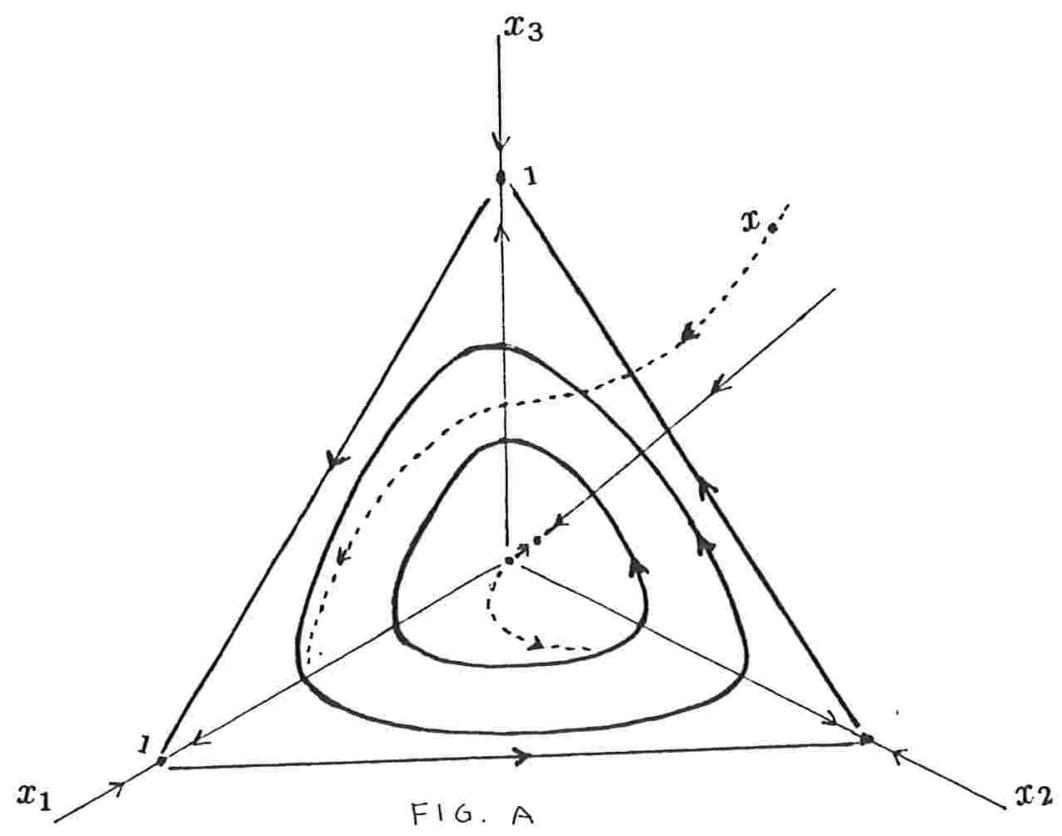

Segundo caso: $\alpha+\beta>2$ e $0<\alpha<1<\beta$

Descreveremos inicialmente a dinâmica do sistema na fronteira de $\Re_{+}^{3}$.

Sabemos que para cada $i=1,2,3$ o conjunto $\left\{\left(x_{1}, x_{2}, x_{3}\right) \in \Re_{+}^{3}: x_{i}=0\right\}$ é invariante. A natureza dos pontos críticos $(0,0,0),(1,0,0),(0,1,0)$ e $(0,0,1)$ é a mesma do caso $\alpha+\beta=2,0<\alpha<1, \beta>1$. 
Consideremos, por exemplo, o sistema na parte do plano coordenado $x_{3}=0 \mathrm{com}$ $x_{i} \geq 0, i=1,2$.

$$
\left\{\begin{array}{l}
\dot{x_{1}}=x_{1}\left(1-x_{1}-\alpha x_{2}\right) \\
\dot{x_{2}}=x_{2}\left(1-\beta x_{1}-x_{2}\right)
\end{array}\right.
$$

Seja $x(t)=\left(x_{1}(t), x_{2}(t)\right)$ a solução que no instante $t=0$ passa por um ponto dado $x=\left(x_{1}^{0}, x_{2}^{0}\right)$ interior a $\Re_{+}^{2}$ e $S(t)=x_{1}(t)+x_{2}(t)$.

Como

$$
\dot{S}=\dot{x_{1}}+\dot{x_{2}}=x_{1}+x_{2}-\left[x_{1}^{2}+x_{2}^{2}+(\alpha+\beta) x_{1} x_{2}\right]<S(1-S)
$$

então o segmento de reta $x_{1}+x_{2}=1, x_{3}=0, x_{i} \geq 0, i=1,2$ não é invariante, pois o campo sobre este segmento aponta para o interior da região $\left\{\left(x_{1}, x_{2}\right) \in \Re_{+}^{2}: x_{1}+x_{2} \leq 1\right\}$.

O comportamento do campo em diferentes regióes do plano $x_{1} x_{2}$ é indicado abaixo.

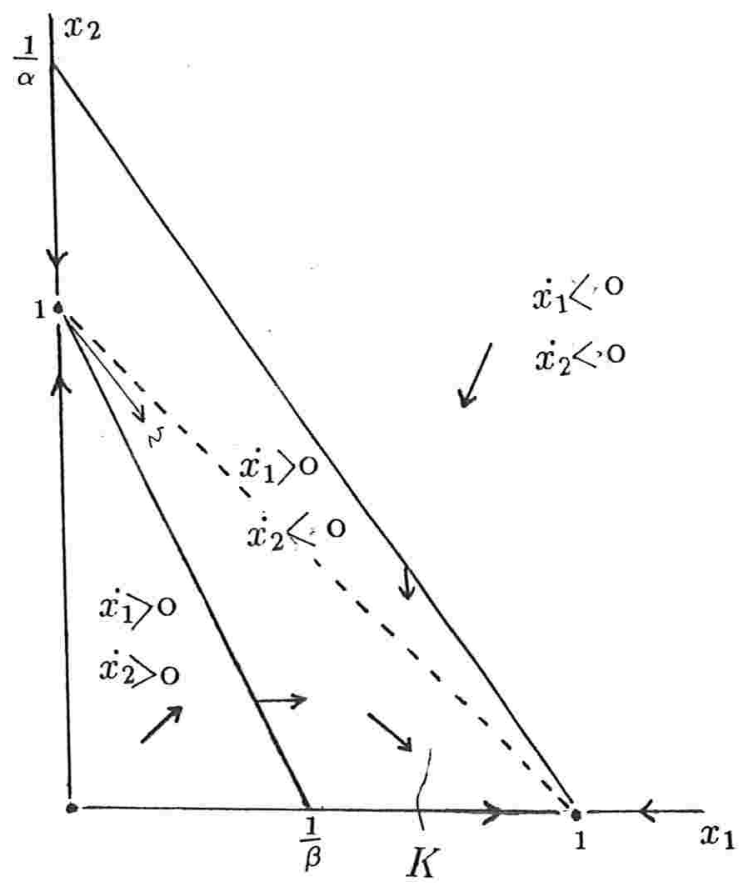

Pode-se observar por exemplo que a região

$$
K=\left\{\left(x_{1}, x_{2}\right) \in \Re_{+}^{2}: 1 \leq \beta x_{1}+x_{2}, x_{1}+x_{2} \leq 1\right\}
$$

é positivamente invariante.

A matriz $D F(0,1)$ tem $v=(\alpha-2, \beta)$ como autovetor correspondente ao autovalor $1-\alpha>0$. Como $(2-\alpha,-\beta) \cdot(1,1)=2-(\alpha+\beta)<0,(2-\alpha,-\beta) \cdot(\beta, 1)=\beta(1-\alpha)>0$, então a variedade instável $\mathcal{O}_{3}$ do ponto $(0,1)$ fica no interior da região $K$. Como $K$ só 
contém dois pontos de equilíbrio: $(0,1),(1,0)$ e não contém órbitas periódicas, então a órbita $\mathcal{O}_{3}$ com $\alpha$-limite o ponto $(0,1)$ tem como $\omega$-limite o ponto $(1,0) . \mathcal{O}_{3}$ é uma curva separatriz, decrescente e de concavidade para cima, já que

$$
\begin{gathered}
\frac{d x_{2}}{d x_{1}}=\frac{x_{2}\left(1-\beta x_{1}-x_{2}\right)}{x_{1}\left(1-x_{1}-\alpha x_{2}\right)}<0 \quad \mathrm{e} \\
\frac{d^{2} x_{2}}{d x_{1}^{2}}=\frac{1}{\left[x_{1}\left(1-x_{1}-\alpha x_{2}\right)\right]^{2}}\left[x_{2}\left(x_{2}-1\right)\left(1-x_{1}-\alpha x_{2}\right)+x_{1} x_{2}\left(1-\beta x_{1}-x_{2}\right)\right]<0 .
\end{gathered}
$$

Assim um esboço do retrato de fase do campo na parte do plano $x_{1} x_{2} \operatorname{com} x_{i} \geq 0$, $i=1,2$ é o seguinte

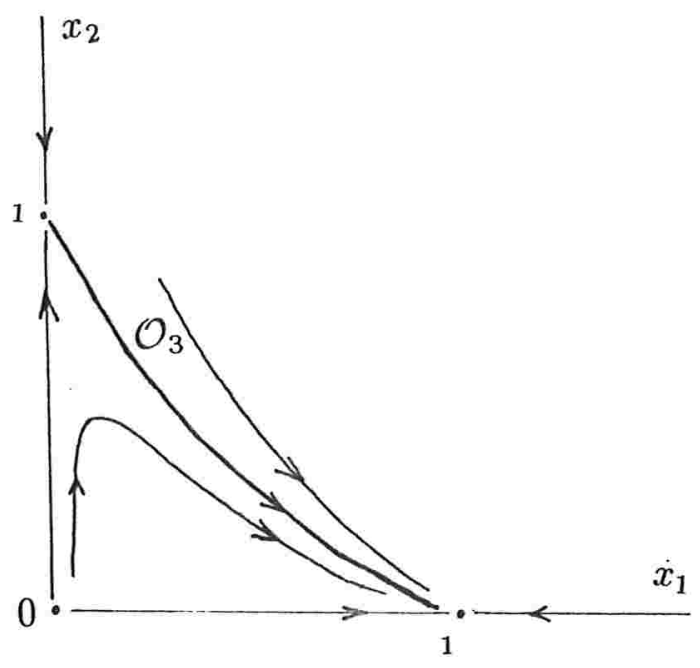

Analisando do mesmo modo nas outras componentes da fronteira de $\Re_{+}^{3}$ tem-se: uma separatriz $\mathcal{O}_{2}$ no plano $x_{2}=0$ de $(1,0,0)$ a $(0,0,1)$ e uma separatriz $\mathcal{O}_{1}$ no plano $x_{1}=0$ de $(0,0,1)$ a $(0,1,0)$.

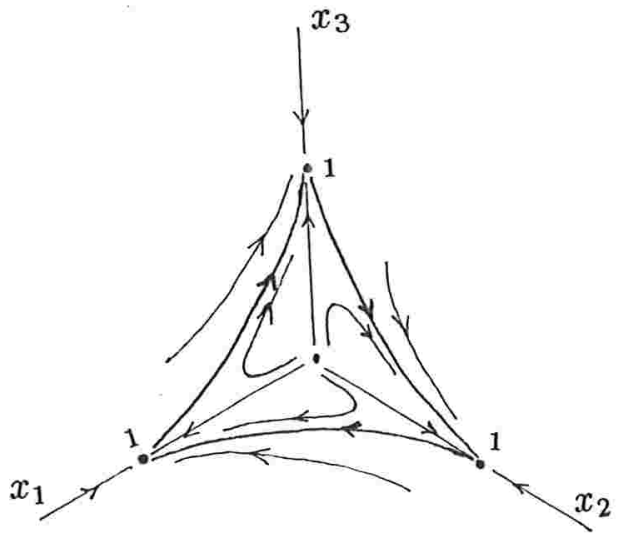


Mostraremos que todos os pontos do interior de $\Re_{+}^{3}$ com exceção dos pontos da semi-reta $x_{1}=x_{2}=x_{3}$ tem como $\omega$-limite o ciclo $\overline{\mathcal{O}_{1} \cup \mathcal{O}_{2} \cup \mathcal{O}_{3}}$.

Seja $x$ um tal ponto, $x(t)=\left(x_{1}(t), x_{2}(t), x_{3}(t)\right)$ a solução que no instante $t=0$ passa por $x$ e $S(t)=x_{1}(t)+x_{2}(t)+x_{3}(t)$.

Tem-se que

$$
\begin{gathered}
\dot{S}=x_{1}+x_{2}+x_{3}-\left[x_{1}^{2}+x_{2}^{2}+x_{3}^{2}+(\alpha+\beta)\left(x_{1} x_{2}+x_{2} x_{3}+x_{1} x_{3}\right)\right] \\
\dot{S}<S(1-S) \quad(\text { pois } \alpha+\beta>2)
\end{gathered}
$$

de onde segue que $\dot{S}(t)<0$ se $S(t) \geq 1$.

Para $x$ tal que $S(0)=1, O_{+}(x)$ entra no interior de $H^{-}$, já que $\dot{S}(0)<0$.

Para $x$ tal que $S(0)=x_{1}+x_{2}+x_{3}>1$. Afirmamos que $O_{+}(x)$ entra no interior do conjunto $H^{-}=\left\{\left(x_{1}, x_{2}, x_{3}\right) \in \Re_{+}^{3}: 0 \leq x_{1}+x_{2}+x_{3} \leq 1\right\}$. De fato: como $S(t)$ decresce enquanto $S(t)>1$, se $\lim _{t \rightarrow+\infty} S(t)=c \geq 1$ então $\omega(x)$ estaria contido no plano $x_{1}+x_{2}+x_{3}=c$ onde $\dot{S}<0$, o que leva a uma contradição, pois $\omega(x)$ é invariante.

Para $x$ tal que $0<S(0)=k<1$ estudaremos o sinal de $\dot{S}(0)$. Como, de (6),

$$
\dot{S}(0)=k-E(x)
$$

onde $E\left(x_{1}, x_{2}, x_{3}\right)=x_{1}^{2}+x_{2}^{2}+x_{3}^{2}+(\alpha+\beta)\left(x_{1} x_{2}+x_{2} x_{3}+x_{1} x_{3}\right)$, então, basta identificar a quádrica $E\left(x_{1}, x_{2}, x_{3}\right)=k$.

Temos que $E\left(x_{1}, x_{2}, x_{3}\right)=\left(x_{1}, x_{2}, x_{3}\right) A\left(x_{1}, x_{2}, x_{3}\right)^{t}$ onde

$$
A=\left[\begin{array}{ccc}
1 & \frac{\alpha+\beta}{2} & \frac{\alpha+\beta}{2} \\
\frac{\alpha+\beta}{2} & 1 & \frac{\alpha+\beta}{2} \\
\frac{\alpha+\beta}{2} & \frac{\alpha+\beta}{2} & 1
\end{array}\right]
$$

com autovalores $\lambda_{1}=1+\alpha+\beta>0, \lambda_{2}=\lambda_{3}=1-(\alpha+\beta)<0$ e autovetores

$$
\begin{aligned}
& u_{1}=\frac{1}{\sqrt{3}}(1,1,1) \text { correspondente a } \lambda_{1} \\
& u_{2}=\frac{1}{\sqrt{2}}(1,-1,0) \text { e } u_{1} \times u_{2}=\frac{1}{\sqrt{6}}(1,1,-2) \text { correspondentes a } \lambda_{2}=\lambda_{3} .
\end{aligned}
$$

Na nova base ortonormal $\left\{u_{1}, u_{2}, u_{3}\right\}$ de $\Re^{n}$, a quádrica $E(x)=k$ tem por equação

$$
\lambda_{1} x_{1}^{\prime 2}+\lambda_{2}{x_{2}^{\prime 2}}^{2}+\lambda_{3} x_{3}^{\prime 2}=k \text {. }
$$


Portanto ela é um hiperbolóide de duas folhas com centro $(0,0,0)$ e cujo eixo de rotação é a reta $x_{1}=x_{2}=x_{3}$. A folha que intercepta $\Re_{+}^{3} \backslash\{0\}$ tem como vértice o ponto $(\bar{x}, \bar{x}, \bar{x})$ com $\bar{x}=\sqrt{\frac{k}{3(1+\alpha+\beta)}}$ e corta os eixos coordenados nos pontos $(\sqrt{k}, 0,0),(0, \sqrt{k}, 0),(0,0, \sqrt{k})$. Tendo em vista que

$$
\begin{gathered}
3 \bar{x}>k \Leftrightarrow 0<k<\frac{3}{1+\alpha+\beta} \\
3 \bar{x}=k \Leftrightarrow k=\frac{3}{1+\alpha+\beta}
\end{gathered}
$$

podemos afirmar que para $0<k \leq \frac{3}{1+\alpha+\beta}$ o vértice da quádrica pertence à região $x_{1}+$ $x_{2}+x_{3} \geq k$.

Uma visualização plana desta análise é dada nas seguintes figuras.
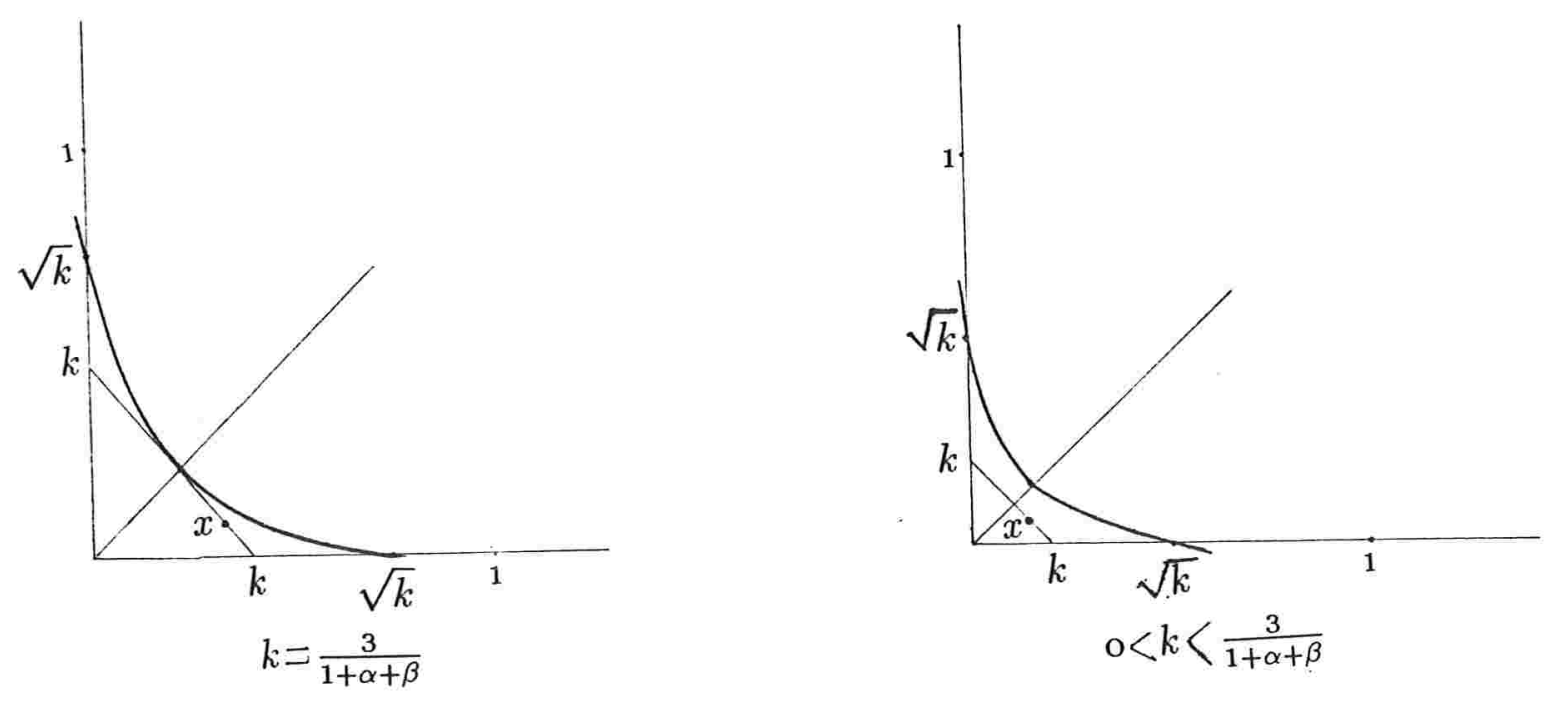

Assim mostramos que para $x$ tal que $0<S(0)=k \leq \frac{3}{1+\alpha+\beta}$, exceto eventualmente $x=(\bar{x}, \bar{x}, \bar{x})$, tem-se $E(x)<k$ e, portanto, $\dot{S}(0)>0$. Logo, podemos afirmar que $S(t)$ cresce enquanto $S(t) \leq \frac{3}{1+\alpha+\beta}$ e que a região

$$
N=\left\{\left(x_{1}, x_{2}, x_{3}\right) \in \Re_{+}^{3}: \frac{3}{1+\alpha+\beta} \leq x_{1}+x_{2}+x_{3} \leq 1\right\}
$$


é positivamente invariante. Além disso analogamente ao caso $S(0) \geq 1$ podemos mostrar que a trajetória entra no interior de $N$.

Concluímos acima que a trajetória de qualquer ponto $x \in \operatorname{int} \Re_{+}^{3}$ fora da reta $x_{1}=$ $x_{2}=x_{3}$ entra no interior da região positivamente invariante $N$ e, portanto, $\omega(x) \subset N$.

Veremos que $\overline{\mathcal{O}_{1} \cup \mathcal{O}_{2} \cup \mathcal{O}_{3}}$ está contido na fronteira de $N$. De fato:

a órbita $\mathcal{O}_{3}$ está contida no interior da região $N_{3}=\left\{\left(x_{1}, x_{2}\right) \in \Re_{+}^{2}: \frac{3}{1+\alpha+\beta} \leq x_{1}+x_{2} \leq 1\right\}$, já que $N_{3}$ é positivamente invariante e que, como já vimos, o vetor $v=(\alpha-2, \beta)$ aponta para o interior de $N_{3}$;

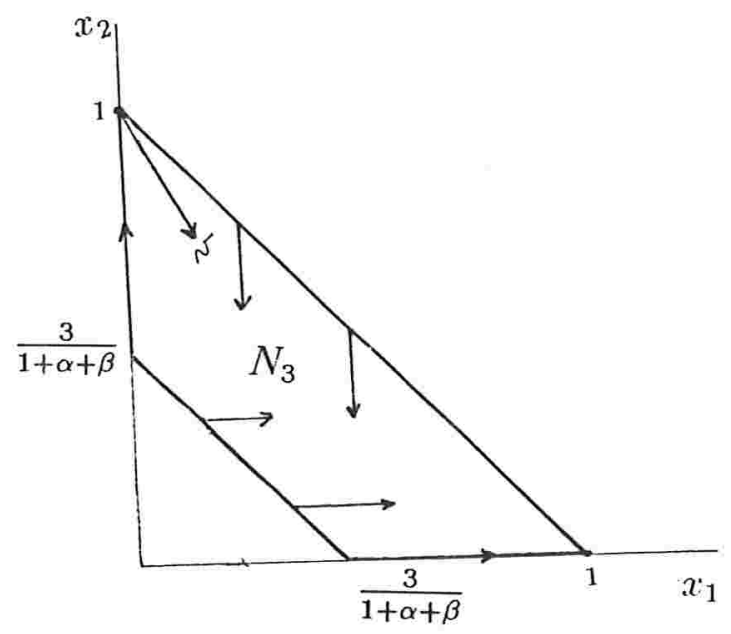

analogamente a órbita $\mathcal{O}_{2}$ está contida no interior da região $N_{2}=\left\{\left(x_{1}, x_{3}\right) \in \Re_{+}^{2}: \frac{3}{1+\alpha+\beta} \leq\right.$ $\left.x_{1}+x_{3} \leq 1\right\}$ e $\mathcal{O}_{1}$ no interior de $N_{1}=\left\{\left(x_{2}, x_{3}\right) \in \Re_{+}^{2}: \frac{3}{1+\alpha+\beta} \leq x_{2}+x_{3} \leq 1\right\}$.

Finalmente mostraremos que, para $x \in$ int $\Re_{+}^{3}$ com exceção dos pontos da reta $x_{1}=x_{2}=x_{3}, \omega(x)=\overline{\mathcal{O}_{1} \cup \mathcal{O}_{2} \cup \mathcal{O}_{3}}$.

Como $P(t)=x_{1}(t) x_{2}(t) x_{3}(t)$ satisfaz a equação

$$
\dot{P}=P\left[3-(1+\alpha+\beta)\left(x_{1}+x_{2}+x_{3}\right)\right]=P[3-(1+\alpha+\beta) S],
$$

então $P(t)$ é decrescente enquanto $S(t)>\frac{3}{1+\alpha+\beta}$. Sabemos que a trajetória entra no 
interior de $N$, portanto, $P(t)$ é decrescente quando $t>t_{0}$ para algum $t_{0}>0$.

Mostraremos que $\lim _{t \rightarrow+\infty} P(t)=0$.

Sabemos que $\dot{P}=P a(t)$ onde $a(t)=3-(1+\alpha+\beta) S(t)$, e que existe $\epsilon=\epsilon(x)>0$ tal que $S(t)>\frac{3}{1+\alpha+\beta}+\epsilon$ quando $t \geq t_{1}$ para algum $t_{1}>t_{0}$. Logo

$$
a(t)=3-(1+\alpha+\beta) S(t) \leq-(1+\alpha+\beta) \epsilon<0 \text { para } t \geq t_{1},
$$

e portanto,

$$
0 \leq P(t)=P\left(t_{1}\right) e^{\int_{t_{1}}^{t} a(r) d r} \leq P\left(t_{1}\right) e^{-(1+\alpha+\beta)\left(t-t_{1}\right) \epsilon}
$$

para $t \geq t_{1}$, de onde segue que $\lim _{t \rightarrow+\infty} P(t)=0$.

Assim mostramos que $x(t)$ se aproxima da fronteira de $\Re_{+}^{3}$. Portanto $\omega(x) \subset N \cap$ Front $\Re_{+}^{3}$, já que $\omega(x) \subset N$.

Como as únicas órbitas inteiramente contidas em $N \cap$ Front $\Re_{+}^{3}$ são as de $\overline{\mathcal{O}_{1} \cup \mathcal{O}_{2} \cup \mathcal{O}_{3}}$, então $\omega(x) \subset \overline{\mathcal{O}_{1} \cup \mathcal{O}_{2} \cup \mathcal{O}_{3}}$.

O conjunto $\omega(x)$ não pode coincidir com nenhum dos pontos de equilíbrio $(1,0,0),(0,1,0)$ e $(0,0,1)$, pois a variedade estável de cada um deles está contida inteiramente num plano coordenado.

Um conjunto $\omega$-limite compacto que contenha alguma órbita regular obedece a seguinte propriedade: "Não existe subconjunto $\emptyset \neq U \varsubsetneqq \omega(x), U$ aberto de $\omega(x)$ tal que $\Phi_{T}(\bar{U}) \subset U$ para algum $T>0$ " (ver apêndice, teorema 2 ).

Temos que o único conjunto invariante em $N \cap$ Front $\Re_{+}^{3}$ que cumpre essa propriedade é o ciclo $\overline{\mathcal{O}_{1} \cup \mathcal{O}_{2} \cup \mathcal{O}_{3}}$. De fato: os conjuntos $\overline{\mathcal{O}_{1}}, \overline{\mathcal{O}_{2}}, \overline{\mathcal{O}_{3}}, \overline{\mathcal{O}_{2} \cup \mathcal{O}_{3}}, \overline{\mathcal{O}_{1} \cup \mathcal{O}_{3}}$ e $\overline{\mathcal{O}_{3} \cup \mathcal{O}_{2}}$ não cumprem a propriedade enunciada (ver figura abaixo).
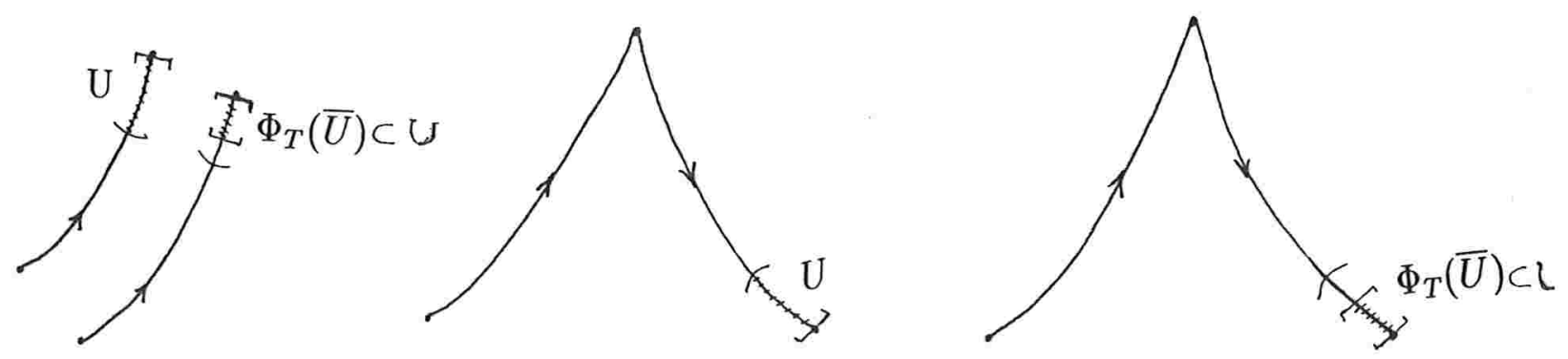
Donde concluímos que $\omega(x)=\overline{\mathcal{O}_{3} \cup \mathcal{O}_{2} \cup \mathcal{O}_{3}}$.

Assim provamos que cada ponto do int $\Re_{+}^{3}$, com exceção dos pontos da semi-reta $x_{1}=x_{2}=x_{3}, x_{i}>0, i=1,2,3$ que convergem para $\frac{1}{1+\alpha+\beta}(1,1,1)$, tem como $\omega$-limite o ciclo $\overline{\mathcal{O}_{3} \cup \mathcal{O}_{2} \cup \mathcal{O}_{3}}$.

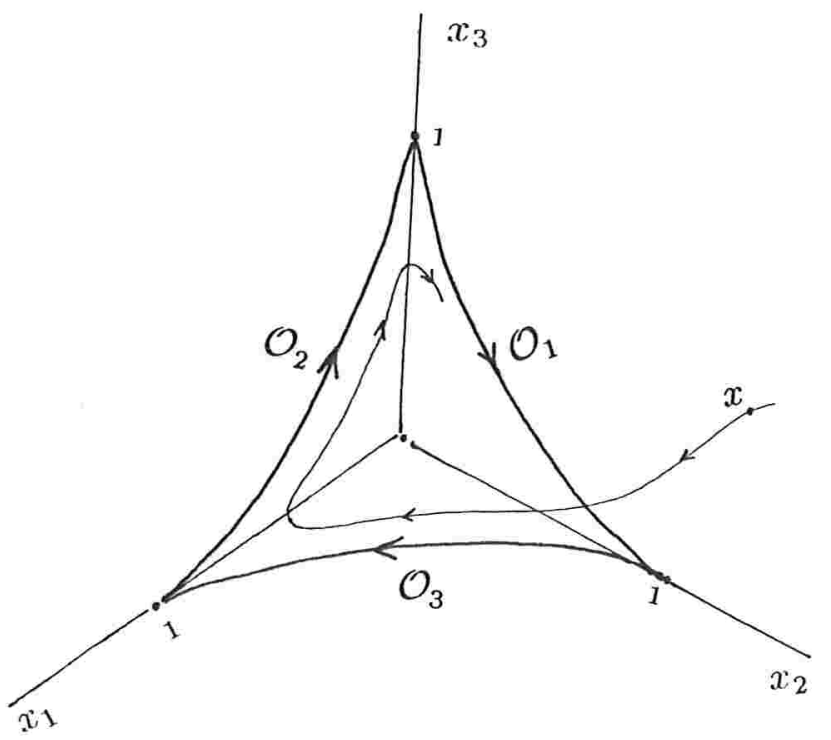




\section{APÊNDICE}

TEOREMA 1. [15] Seja $S$ um espaço métrico, $E \subset S$ e $f: E \rightarrow \Re$ uma função que satisfaz a condição de Lipchitz

$$
|f(x)-f(y)| \leq M|x-y|
$$

em $E$. Então $f$ admite uma extensão lipchitziana em $S$ com a mesma constante $M$ de Lipchitz.

Demonstração. Sejam $x, y \in E$, pela validade de (1) tem-se que

$$
f(y)-M|y-x| \leq f(x) .
$$

Definimos a função $\Phi: S \rightarrow \Re \cup\{+\infty\}$ dada por

$$
\Phi(x)=\sup \{f(y)-M|y-x|: y \in E\} .
$$

Se $x \in E$, de (2) segue que $\Phi(x) \leq f(x)$; como $f(y)-M|y-x|=f(x)$ para $y=x$, então $\Phi(x)=f(x)$; assim, $\Phi$ é uma extensão de $f$.

Sejam $x, x^{\prime} \in S, \operatorname{como} \sup (A+B) \leq \sup A+\sup B$ então

$$
\Phi(x) \leq \sup \left\{M\left(\left|y-x^{\prime}\right|-|y-x|\right): y \in E\right\}+\Phi\left(x^{\prime}\right) \leq M\left|x-x^{\prime}\right|+\Phi\left(x^{\prime}\right)
$$

Se $x^{\prime} \in E$, de (3) segue que $\Phi(x) \in \Re$ (já que $\Phi\left(x^{\prime}\right) \in \Re$ ) para todo $x \in S$.

Dados $x, x^{\prime} \in S$ suponhamos que $\Phi\left(x^{\prime}\right) \leq \Phi(x)$, então temos de (3) que

$$
\left|\Phi(x)-\Phi\left(x^{\prime}\right)\right| \leq M\left|x-x^{\prime}\right|
$$

de onde segue que $\Phi$ é lipchitziana em $S$, com constante de Lipchitz $M$.

COROLÁRIO Seja $S$ um espaço métrico, $E \subset S$ e $f: E \rightarrow \Re^{n}$ uma função lipchitziana em $E$. Então $f$ admite uma extensão lipchitziana em $S$.

\section{TEOREMA 2. [2]}

Sejam $F: W \rightarrow \Re^{n}$ um campo de classe $C^{1}$ num aberto $W \subset \Re^{n}$ e $L$ um conjunto $\omega$-limite compacto contendo alguma órbita regular. Então, não existe $\emptyset \neq U \varsubsetneqq L, U$ aberto de $L$, tal que $\Phi_{T}(\bar{U}) \subset U$ para algum $T>0$. 
Para demonstrar este teorema precisaremos do seguinte:

LEMA Nas mesmas hipóteses do teorema, se existir $\emptyset \neq U \subseteq L, U$ aberto de $L$, tal que $\Phi_{T}(\bar{U}) \subset U$ para algum $T>0$, então o conjunto

$$
U^{\prime}=\bigcup_{t \geq 0} \Phi_{t}(U)=\bigcup_{0 \leq t \leq T} \Phi_{t}(U)
$$

é um aberto próprio de $L$, positivamente invariante, tal que $\Phi_{T}\left(\overline{U^{\prime}}\right) \subset U^{\prime}$.

Notação : $\Phi_{t}(x)=x \cdot t, \Phi_{t}(U)=U \cdot t$.

\section{Demonstração do lema.}

i) Mostraremos que $U^{\prime}=\bigcup_{t \geq 0} \Phi_{t}(U)$ é aberto de $L$.

Dado $t>0$, como $L$ é compacto, existe um aberto $V$ em $W, V \supset L$ onde o fluxo $\Phi_{s}$ está definido para $s \in[0, t]$.

Como $U$ é aberto em $L$, então existe $D \subset V$ aberto de $W$ tal que $U=L \cap D$. Sendo $\Phi_{t}: L \rightarrow L$ uma bijeção tem-se que

$$
\Phi_{t}(U)=\Phi_{t}(L \cap D)=\Phi_{t}(L) \cap \Phi_{t}(D)=L \cap \Phi_{t}(D)
$$

e como $\Phi_{t}(D)$ é aberto de $W$, conclui-se que $\Phi_{t}(U)$ é aberto em $L$. Assim, $U^{\prime}$ é aberto de $L$.

ii) $U^{\prime}$ é positivamente invariante, já que se $z \in U^{\prime}, z=\Phi_{t}(x)$ para algum $x \in U$ e $t \geq 0$, então para $s \geq 0$

$$
\Phi_{s}(z)=\Phi(s+t, x) \in \Phi_{s+t}(U) \subset U^{\prime}
$$

iii) Mostrando que $\bigcup_{t \geq 0} \Phi_{t}(U)=\bigcup_{0 \leq t \leq T} \Phi_{t}(U)$.

É trivial que $\bigcup_{t \geq 0} \Phi_{t}(U) \supset \bigcup_{0 \leq t \leq T} \Phi_{t}(U)$.

Sejam $z=\Phi_{t}(x) \in \bigcup_{t \geq 0} \Phi_{t}(U) \operatorname{com} t>T$ e $n \geq 1, n \in N$ tais que $n T \leq t \leq(n+1) T$. Como $t=n T+s$ com $0 \leq s \leq T, z=\Phi(s+n T, x)=\Phi(s, \Phi(n T, x)) \in \Phi_{s}(U)$, já que $\Phi(n T, x) \in U$, pois $\Phi_{T}(U) \subset U$. Donde $z \in \bigcup_{0 \leq t \leq T} \Phi_{t}(U)$ e, portanto, $\bigcup_{t \geq 0} \Phi_{t}(U) \subset$ $\bigcup_{0 \leq t \leq T} \Phi_{t}(U)$. 
iv) Mostrando que $\Phi_{T}\left(\overline{U^{\prime}}\right) \subset U^{\prime}$.

Basta mostrar que $\overline{U^{\prime}} \subset \bigcup_{0 \leq t \leq T} \Phi_{t}(\bar{U})$, daí tendo em conta que $\Phi_{T}(\bar{U}) \subset U$ segue que $\Phi_{T}\left(\overline{U^{\prime}}\right) \subset \bigcup_{0 \leq t \leq T} \Phi_{T}\left(\Phi_{t}(\bar{U})\right)=\bigcup_{0 \leq t \leq T} \Phi_{t}\left(\Phi_{T}(\bar{U})\right) \subset \bigcup_{0 \leq t \leq T} \Phi_{t}(U)=U^{\prime}$.

Dado $z \in \overline{U^{\prime}}$, sejam $0 \leq t_{n} \leq T$ e $x_{n} \in U$ tais que $z=\lim _{n \rightarrow+\infty} \Phi\left(t_{n}, x_{n}\right)$. Como $[0, T]$ e $\bar{U}$ são compactos podemos supor que

$$
t_{n} \rightarrow \bar{t} \in[0, T] \text { e } x_{n} \rightarrow \bar{x} \in \bar{U}
$$

assim,

$$
z=\lim _{n \rightarrow+\infty} \Phi\left(t_{n}, x_{n}\right)=\Phi(\bar{t}, \bar{x}) \in \Phi_{\bar{t}}(\bar{U}) \subset \bigcup_{0 \leq t \leq T} \Phi_{t}(\bar{U})
$$

portanto $\overline{U^{\prime}} \subset \bigcup_{0 \leq t \leq T} \Phi_{t}(\bar{U})$

v) Mostrando que $U^{\prime}$ é um subconjunto próprio de $L$.

Suponhamos que não fosse, então $U^{\prime}=L$.

Afirmamos que $L-\bar{U} \neq \emptyset$. De fato: se $\bar{U}=L$, como $\Phi_{T}(\bar{U}) \subset U$ teríamos $L=\Phi_{T}(L) \subset U$ que contraria a hipótese de que $U q L$.

Dados $x_{0} \in L-\bar{U}$ afirmamos que $x_{i}=\Phi_{i T}\left(x_{0}\right) \in L-U$ para $i=-1,-2, \ldots$ De fato: suponhamos que para algum $i, x_{i} \in U$, então $\Phi_{T}\left(x_{i}\right) \in \Phi_{T}(U) \subset \Phi_{T}(\bar{U}) \subset U$, de onde segue que

$$
x_{0}=\Phi_{(-i-1) T}\left(\Phi_{T}\left(x_{i}\right)\right) \in U
$$

o que contraria o fato $x_{0} \notin U$.

A órbita de $x_{0}$ entre $x_{i}$ e $x_{i+1}$ inclusive entre $x_{0}$ e $x_{-1}$ corta $U \cdot T$, já que como $x_{i} \in U^{\prime}-U$ podemos escrever $x_{i}=y_{i} \cdot t_{i} \operatorname{com} y_{i} \in U$ e $0<t_{i}<T$ e, portanto $y_{i} \cdot T \in U \cdot T$ é um ponto entre $x_{i}$ e $x_{i+1}$.

Para cada $p \in L-U$ seja $\alpha(p)>0$ a quantidade mínima de tempo que a trajetória de $p$ entre $p$ e $\Phi_{-T}(p)$ gasta para atravessar $U-\bar{U} \cdot T$ quando passa por $\bar{U} \cdot T$.

\section{Consideremos}

$$
\alpha=\inf \{\alpha(p) / p \in L-U\}
$$

afirmamos que $\alpha>0$. De fato : em caso contrário existiria uma seqüência de pontos $p_{n} \in$ $L-U$ tal que $\alpha\left(p_{n}\right) \rightarrow 0$. Como $L-U$ é compacto, podemos supor que $p_{n} \rightarrow p \in L-U$; como $\alpha(p)>0$ e $d(L-U, \bar{U} \cdot T)>0$ teríamos pela continuidade do fluxo que $\alpha\left(p_{n}\right) \nrightarrow 0$. 
Mostraremos agora que para cada $i$, a quantidade de tempo $t_{i}$ que a órbita de $x_{0}$ entre $x_{i}$ e $x_{i+1}$ gasta em $U \cdot T$ é menor que $T+2 i \alpha$, o que leva a um absurdo, daí segue-se que $U^{\prime} \mp L$.

A quantidade de tempo $t_{1}$ que a órbita de $x_{0} O\left(x_{0}\right)$ entre $x_{-1}$ e $x_{0}$ gasta em $U \cdot T$ é menor que $T-2 \alpha$, isto é, $t_{1}<T-2 \alpha$. Como a quantidade de tempo $\bar{t}$ que $O\left(x_{0}\right)$ entre $x_{-2}$ e $x_{-1}$ gasta em $U$ é menor ou igual a $t_{1}$ e a quantidade de tempo $t_{2}$ que $O\left(x_{0}\right)$ entre $x_{-2}$ e $x_{-1}$ gasta em $U \cdot T$ é menor ou igual a $\bar{t}-2 \alpha$, então $t_{2}<T-4 \alpha$.

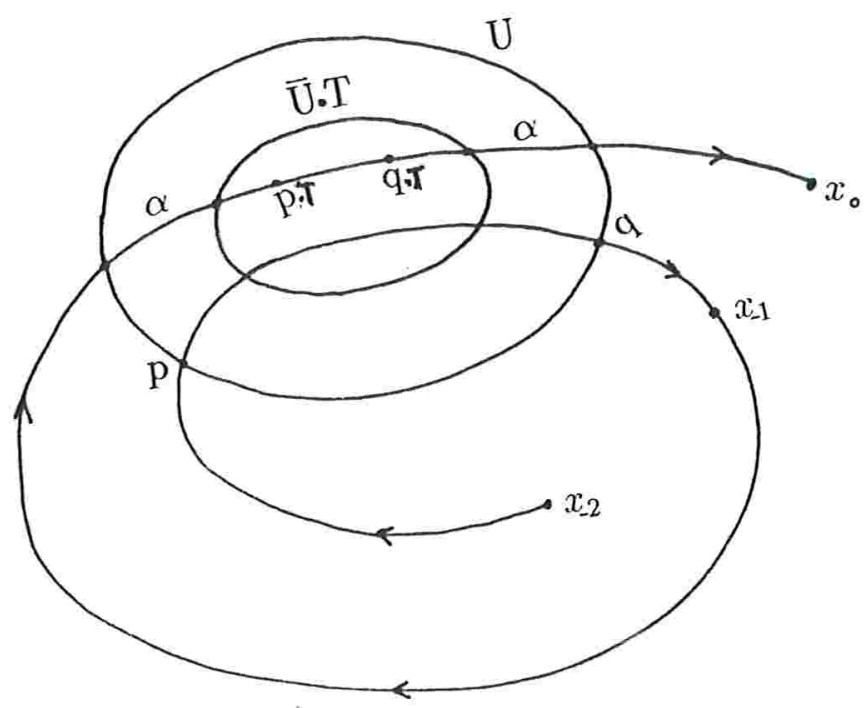

E assim sucessivamente concluímos que a quantidade de tempo que $O\left(x_{0}\right)$ entre $x_{i}$ e $x_{i+1}$ gasta em $U \cdot T$ é menor que $T+2 i \alpha$.

\section{Demonstração do teorema 2.}

Suponhamos que exista $U \neq \emptyset, U$ aberto de $L, U \nsubseteq L$ tal que $\Phi_{T}(\bar{U}) \subset U$ para algum $T>0$. Então pelo lema anterior o conjunto $U^{\prime}=\bigcup_{t \geq 0} \Phi_{t}(U)=\bigcup_{0 \leq t \leq T} \Phi_{t}(U)$ é aberto de $L$, positivamente invariante, $U^{\prime} \varsubsetneqq L$ e $\Phi_{T}\left(\overline{U^{\prime}}\right) \subset U^{\prime}$.

Podemos ver que $\overline{U^{\prime}} \varsubsetneqq L$, já que de outro modo se $\overline{U^{\prime}}=L$, como $L=\Phi_{T}(L)$ e $\Phi_{T}\left(\bar{U}^{\prime}\right) \subset U^{\prime}$ teríamos $L \subset U^{\prime}$ que contraria o fato $U^{\prime} \mp L$. 
Sejam $y \in L-\overline{U^{\prime}}$ e $L=\omega(x)$ com $x \in W$, mostraremos que existem $a>0$ e $r>0$ tais que $d(x \cdot t, y)>\frac{a}{2}>0$ para todo $t \geq r>0$ o que implica que $y \notin \omega(x)=L$. Donde segue o teorema por contradição.

Sejam $a=d\left(y, \overline{U^{\prime}}\right)>0, \quad b=d\left(L-U^{\prime}, \overline{U^{\prime}} \cdot T\right)>0$; como o fluxo $\Phi_{t}$ é contínuo no compacto $[0, T] \times \overline{O_{+}(x)}$, existe $\gamma>0$ tal que:

se $d(p, q)<\gamma \operatorname{com} p, q \in \overline{O_{+}(x)}$, então

$$
d(p \cdot t, q \cdot t)<\frac{1}{2} \min \{a, b\} \text { para todo } 0 \leq t \leq T .
$$

Afirmamos que existe $s>0$ tal que $d(x \cdot t, \omega(x))<\gamma$ para todo $t \geq s$.

De fato: no caso contrário, para todo $n>0$, existe $t_{n} \geq n$ tal que $d\left(x \cdot t_{n}, \omega(x)\right) \geq \gamma$; como $\overline{O_{+}(x)}$ é compacto e $x \cdot t_{n} \in \overline{O_{+}(x)}$, pode-se supor que $x \cdot t_{n} \rightarrow \bar{x} \in \omega(x)$, assim $0=d(\bar{x}, \omega(x)) \geq \gamma$ o que contraria o fato $\gamma>0$.

$\left(^{*}\right)$ Sejam $z \in U^{\prime} \subset \omega(x)$ e $r>s$ tais que $d(x \cdot r, z)<\gamma$. Por (1) tem-se que

$$
d(x \cdot(r+t), z \cdot t)<\frac{1}{2} \min \{a, b\} \text { para } 0 \leq t \leq T
$$

e tendo em conta que $z \cdot t \in U^{\prime} \subset \overline{U^{\prime}}$ temos

$$
a \leq d(y, z \cdot t) \leq d(y, x \cdot(r+t))+d(x \cdot(r+t), z \cdot t)<d(x \cdot(r+t), y)+\frac{a}{2}
$$

o que implica que

$$
d(x \cdot(r+t), y)>\frac{a}{2} \text { para } 0 \leq t \leq T .
$$

(**) Sabemos por (2) que $d(x \cdot(r+T), \omega(x))<\gamma$, logo existe algum $z_{1} \in \omega(x)$ tal que $d\left(x \cdot(r+T), z_{1}\right)<\gamma$. Mostraremos que escolhendo $\gamma<\frac{b}{2}, z_{1} \in U^{\prime}$.

Seja $\bar{z} \in \omega(x)-U^{\prime} ;$ como $z \cdot T \in \overline{U^{\prime}} \cdot T$ e, por $(3), d(x \cdot(r+T), z \cdot T)<\frac{b}{2}$, então

$$
\begin{gathered}
d\left(z_{1}, \bar{z}\right) \geq d\left(\bar{z}, x \cdot(T+r)-d\left(x \cdot(T+r), z_{1}\right) \geq\right. \\
\geq d(\bar{z}, z \cdot T)-d(z \cdot T, x \cdot(T+r))-d\left(x \cdot(T+r), z_{1}\right) \\
\text { e } \quad d\left(z_{1}, \bar{z}\right)>b-\frac{b}{2}-\frac{b}{2}=0 . \text { Portanto } z_{1} \in U^{\prime} .
\end{gathered}
$$

Assim existe $z_{1} \in U^{\prime}$ tal que $d\left(x \cdot(r+T), z_{1}\right)<\gamma$ e pelo mesmo processo seguido em (*) começando em $x \cdot(r+T)$ e $z_{1}$ (em lugar de $x \cdot r$ e $z$ ) temos que

$$
d(x \cdot(r+T+t), y)>\frac{a}{2} \text { para } 0 \leq t \leq T .
$$


E pelo mesmo processo seguido em $\left(^{* *}\right)$ tendo em conta que $d\left(x \cdot(r+2 T), z_{1} \cdot T\right)<\frac{b}{2}$ e que $z_{1} \cdot T \in \overline{U^{\prime}} \cdot T$ mostra-se que existe $z_{2} \in U^{\prime}$ tal que $d\left(x \cdot(r+2 T), z_{2}\right)<\gamma$.

Continuando sucessivamente do mesmo modo, concluímos que

$$
d(x \cdot t, y)>\frac{a}{2} \text { para todo } t \geq r>0 .
$$




\section{BIBLIOGRAFIA}

[1] D. M. BLOOM. Linear algebra and geometry. Cambridge University Press, 1979.

[2] W. A. COPPEL. Stability and Asymptotic Behavior of Differential Equations. D.C. Heath, Boston, 1969.

[3] J. E. FRANKE AND J. F. SELGRADE. Abstract $\omega$-limits, Chain Recurrent sets, and Basic sets for flows. Proceedings of the American Mathematical Society. Volume 60, 1970, p.p. 309 - 317.

[4] J. S. GRIFFITH. Mathematics of Cellular Control Process II. Positive feedback to one gene. Ibid, 20 (1968), p.p. 209 - 216.

[5] F. R. GANTMACHER. The theory of Matrices. Vol 2, Chelsea, New York, 1959.

[6] P. HARTMAN. Ordinary Differential Equations. John Wiley, New York, 1964.

[7] M. W. HIRSCH. Systems of Differential Equations Which are Competitive or Cooperative. I : Limit sets. Siam J. Math. Anal. Vol 13 - n.o 2, 1982, pp. 167 179.

[8] M. W. HIRSCH. Systems of Differential Equations that are Competitive or Cooperative II : Convergence Almost Everywhere. Siam J. Math. Anal. Vol 16 n.o 3 - 1985. p.p. $423-439$.

[9] M. W. HIRSCH. Stability and Convergence in Strongly Monotone Dynamical Systems. Journal für die reine und angewandte Mathematik. Berlin - New York 1988. p.p. 1 - 53 .

[10] M. W. HIRSCH. The Dynamical Systems Approach to Differential Equations. Bulletin of the American Mathematical Society. Volume 11, n.o 11 - 1984. p.p. 1 - 61.

[11] M. A. KRASNOSELISKI. The Operator of Translation Along the Trajetories of Differential Equations. Translations of Mathematical Monographs, Volume 19. American Mathematical Society Providence, Rhode Island, 1968. 
[12] V. LAKSHMIKANTHAM AND S. LEELA. Differential and Integral Inequalities Theory and Applications Vol I. Academic Press, 1969.

[13] R. M. MAY AND W. J. LEONARD. Nonlinear Aspects of Competition between three species. Siam J. Appl. Math. Vol. 29, n.o 2, 1975, p.p. 243 - 253.

[14] J. MARSDEN AND McCRACKEN. The Hopf Bifurcation and Its Applications. Applied Mathematical Sciences. Vol. 19, Springer - Verlag, New York, 1976.

[15] E. J. McSHANE. Extension of Range of Function . Bull. Amer. Math. Soc., 1934, p.p. $837-842$.

[16] WALDIR MUNIZ OLIVA - G. FUSCO. Jacobi matrices and tranversality. Proceedings of the Royal Society of Edinburgh, 109A, 231 - 243, 1988.

[17] G. FUSCO AND W. MUNIZ OLIVA. Transversality between Invariant Manifolds of Periodic Orbits for a Class of Monotone Dynamical Systems. Reprinted from Journal of Dynamics and Differential Equations. Vol 2, n.o 1, January 1990.

[18] W. MUNIZ OLIVA. Equações Diferenciais Ordinárias. Instituto de Matemática e Estatística da Universidade de São Paulo, 1971.

[19] HAL L. SMITH Periodic Orbits of Competitive and Cooperative Systems. Journal of Differential Equations 65, 1986, p.p. 361 - 373.

[20] S. SMALE. On the Differential Equations of Species in Competitionn. J. MATH. BIO. 3, 1976, p.p. 5 - 7.

[21] J. SOTOMAYOR. Lições de equações diferenciais ordinárias. IMPA - Brasil, 1979.

[22] P. SCHUSTER, K. SIGMUND AND WOLFF. On $\omega$-limits for Competition Between Three Species. Siam J. Appl. Math. Vol. 37, n.o 1, 1979, p.p. 49 - 54. 